In the book "Operator theory and Applications", Fields Institute Communications vol. 25, AMS, Providence, 2000, pp.15-75 (Ed.A.G.Ramm, P.N.Shivakumar, A.V.Strauss).

\title{
Property C for ODE and applications to inverse problems.
}

\author{
A.G. Ramm \\ Mathematics Department, Kansas State University, \\ Manhattan, KS 66506-2602, USA \\ ramm@math.ksu.edu
}

\begin{abstract}
An overview of the author's results is given. Property C stands for completeness of the set of products of solutions to homogeneous linear Sturm-Liouville equations. The inverse problems discussed include the classical ones (inverse scattering on a half-line, on the full line, inverse spectral problem), inverse scattering problems with incomplete data, for example, inverse scattering on the full line when the reflection coefficient is known but no information about bound states and norming constants is available, but it is a priori known that the potential vanishes for $x<0$, or inverse scattering on a half-line when the phase shift of the $s$-wave is known for all energies, no bound states and norming constants are known, but the potential is a priori known to be compactly supported. If the potential is compactly supported, then it can be uniquely recovered from the knowledge of the Jost function $f(k)$ only, or from $f^{\prime}(0, k)$, for all $k \in \Delta$, where $\Delta$ is an arbitrary subset of $(0, \infty)$ of positive Lebesgue measure.

Inverse scattering problem for an inhomogeneous Schrödinger equation is studied.

Inverse scattering problem with fixed-energy phase shifts as the data is studied.

Some inverse problems for parabolic and hyperbolic equations are investigated.

A detailed analysis of the invertibility of all the steps in the inversion procedures for solving the inverse scattering and spectral problems is presented.

An analysis of the Newton-Sabatier procedure for inversion of fixedenergy phase shifts is given.

Inverse problems with mixed data are investigated.

Representation formula for the $I$-function is given and properties of this function are studied.

Algorithms for finding the scattering data from the $I$-function, the $I$-function from the scattering data and the potential from the $I$-function are given.

A characterization of the Weyl solution and a formula for this solution in terms of Green's function are obtained.
\end{abstract}

1991 Mathematics Subject Classification. 34B25, 35R30, 81F05, 81F15, 73D25.

Key words and phrases. Property C, inverse scattering, inverse problems, incomplete data, fixed-energy phase shifts, $I$-function, Weyl function, spectral function. 
Table of Contents

\section{Property $\mathrm{C}$ for $\mathrm{ODE}$}

\section{Applications of property $\mathrm{C}$}

2.1 Uniqueness of the solution to inverse scattering problem with the data $I$-function.

2.2 Uniqueness of the solution to inverse scattering problem on the half-line. 2.3 Compactly supported potentials are uniquely determined by the phase shift of $s$-wave.

2.4 Recovery of $q \in L_{1,1}(\mathbb{R})$ from the reflection coefficient alone.

2.5 Inverse scattering with various data.

3. Inverse problems on a finite interval with mixed data.

4. Property $\mathrm{C}$ and inverse problems for some PDE. Recovery compactly supported potential from the knowledge of $f(k)$ or $f^{\prime}(0, k)$.

5. Invertibility of the steps in the inversion procedure, in the inverse scattering and spectral problems.

6. Inverse problem for an inhomogeneous Schrödinger equation.

7. Inverse scattering problem with fixed-energy data.

7.1 Three-dimensional inverse scattering problem. Property C. Stability estimates.

7.2 Approximate inversion of fixed-energy phase shifts.

8. A uniqueness theorem for inversion of fixed-energy phase shifts.

9. Discussion of the Newton-Sabatier procedure for recovery of $q(r)$ from the fixed-energy phase shifts.

10. Reduction of some inverse problems to an overdetermined Cauchy problem. An iterative method for solving this problem.

11. Representation of $I$-function.

12. Algorithms for finding $q(x)$ from $I(k)$.

13. Remarks.

13.1. Representation of the products of solutions to Schrödinger equation (1.1).

13.2. Characterization of Weyl's solutions.

13.3. Representation of the Weyl solution via the Green function.

Bibliography 


\section{Property C for ODE}

In this paper a review of the author's results is given and some new results are included. The bibliography is not complete. Only the papers and books used in the work on this paper are mentioned. The contents of this paper are clear from the table of contents.

The results presented in this paper include:

1. Property $\mathrm{C}$ for ordinary differential equations (ODE), that is, theorems about completeness of the sets of products of solutions to homogeneous ODE.

2. Uniqueness theorems for finding the potential

a) from the $I$-function (which equals the Weyl function),

b) from the classical scattering data for the half-axis problem (a new very short proof which does not use the Marchenko method),

c) from the phase shift of $s$-wave in the case when the potential $q$ is compactly supported and no bound states or norming constants are known,

d) from the reflection coefficient only (when $q=0$ for $x<x_{0}$ ),

e) from mixed data: part of the set of eigenvalues and the knowledge of $q(x)$ on a part of a finite interval,

f) from overdetermined Cauchy data,

g) from part of the fixed-energy phase shifts,

h) from various type of data which are typical in PDE problems,

i) from $f(k)$ or $f^{\prime}(0, k)$ when $q$ is compactly supported,

j) from the scattering data for a solution to an inhomogeneous Schrödinger equation.

3. Reconstruction algorithms for finding the potential from overdetermined Cauchy data, for finding $f(k)$ and $f^{\prime}(0, k)$ from the scattering data, for finding the scattering data from the $I$-function and the $I$-function from the scattering data.

4. Properties of the $I$-function and a representation formula for it.

5. Stability estimate for the solution of the inverse scattering problem with fixed-energy data. Example of two compactly supported real-valued piecewiseconstant potentials which produce practically the same phase shifts for all values of $\ell$.

6. Discussion of the Newton-Sabatier procedure for inversion of the fixed-energy phase shifts. Proof of the fact that this procedure cannot recover generic potentials, for example, compactly supported potentials.

7. Detailed analysis and proof of the invertibility of each of the steps in the inversion schemes of Marchenko and Gelfand-Levitan.

8. Representation of the Weyl solution via the Green function and a characterization of this solution by its behavior for large complex values of the spectral parameter and $x$ running through a compact set.

Completeness of the set of products of solutions to ODE has been used for inverse problems on a finite interval in the works of Borg [2] and Levitan [12], [13].

Completeness of the set of products of solutions to homogeneous partial differential equations (PDE) was introduced and used extensively under the name property $\mathrm{C}$ in [26]-[31], and [17]. Property $\mathrm{C}$ in these works differs essentially from the property $\mathrm{C}$ defined and used in this paper: while in [26]-[31], and [17] property $\mathrm{C}$ means completeness of the set of products of solutions to homogeneous PDE with 
fixed value of the spectral parameter, in this paper we prove and use completeness of the sets of products of solutions to homogeneous ordinary differential equations (ODE) with variable values of the spectral parameter. Note that the dimension of the null-space of a homogeneous PDE (without boundary conditions) with a fixed value of the spectral parameter is infinite, while the dimension of the null-space of a homogeneous ODE (without boundary conditions) with a fixed value of the spectral parameter is finite. Therefore one cannot have property $\mathrm{C}$ for ODE in the sense of [26]-[31], and [17], because the set of products of solutions to homogeneous ODE with fixed value of the spectral parameter is finite-dimensional.

In this paper property $\mathrm{C}$ for ordinary differential equations is defined, proved and used extensively. Earlier papers are [38] and [18].

Let

$$
\ell u:=u^{\prime \prime}+k^{2} u-q(x) u=0, \quad x \in \mathbb{R}=(-\infty, \infty) .
$$

Assume

$$
q \in L_{1,1}, \quad L_{1, m}:=\left\{q: q=\bar{q}, \int_{-\infty}^{\infty}(1+|x|)^{m}|q(x)| d x<\infty\right\} .
$$

It is known [14], [17] that there is a unique solution (the Jost solution) to (1.1) with the asymptotics

$$
f(x, k)=e^{i k x}+o(1), \quad x \rightarrow+\infty .
$$

We denote $f_{+}(x, k):=f(x, k), f_{-}(x, k):=f(x,-k), k \in \mathbb{R}$. The function $f(0, k)=$ $f(k)$ is called the Jost function. The function $f(k)$ is analytic in $\mathbb{C}_{+}:=\{k: \operatorname{Im} k>$ $0\}$ and has at most finitely many zeros in $\mathbb{C}_{+}$which are located at the points $i k_{j}, k_{j}>0,1 \leq j \leq J$. The numbers $-k_{j}^{2}$ are the negative eigenvalues of the selfadjoint operator defined by the differential expression $L_{q}:=-\frac{d^{2}}{d x^{2}}+q(x)$ and the boundary condition $u(0)=0$ in $L^{2}\left(\mathbb{R}_{+}\right), \mathbb{R}_{+}=(0, \infty)$. The function $f(k)$ may have zero at $k=0$. This zero is simple: if $f(0)=0$ then $\dot{f}(0) \neq 0, \dot{f}:=\frac{\partial f}{\partial k}$.

Let $\varphi$ and $\psi$ be the solutions to (1.1) defined by the conditions

$$
\varphi(0, k)=0, \quad \varphi^{\prime}(0, k)=1 ; \quad \psi(0, k)=1, \quad \psi^{\prime}(0, k)=0,
$$

where $\varphi^{\prime}:=\frac{\partial \varphi}{\partial x}$. It is known [14], [17], that $\varphi(x, k)$ and $\psi(x, k)$ are even entire functions of $k$ of exponential type $\leq|x|$.

Let $g_{ \pm}(x, k)$ be the unique solution to (1.1) with the asymptotics

$$
g_{ \pm}(x, k)=e^{ \pm i k x}+o(1), \quad x \rightarrow-\infty
$$

Definition 1.1 Let $p(x) \in L_{1,1}\left(\mathbb{R}_{+}\right)$and assume

$$
\int_{0}^{\infty} p(x) f_{1}(x, k) f_{2}(x, k) d x=0, \quad \forall k>0,
$$

where $f_{j}(x, k)$ is the Jost solution to (1.1) with $q(x)=q_{j}(x), j=1,2$. If (1.6) implies $p(x)=0$, then we say that the pair $\left\{L_{1}, L_{2}\right\}, L_{j}:=L_{q_{j}}:=-\frac{d^{2}}{d x^{2}}+q_{j}(x)$ has property $C_{+}$.

If $p \in L_{1,1}\left(\mathbb{R}_{-}\right)$and

$$
\int_{-\infty}^{0} p(x) g_{1}(x, k) g_{2}(x, k) d x=0 \quad \forall k>0,
$$

implies $p(x)=0$, then we say that the pair $\left\{L_{1}, L_{2}\right\}$ has property $C_{-}$. 
In (1.7) $g_{j}:=g_{j+}(x, k)$.

Fix an arbitrary $b>0$. Assume that

$$
\int_{0}^{b} p(x) \varphi_{1}(x, k) \varphi_{2}(x, k) d x=0 \quad \forall k>0
$$

implies $p(x)=0$. Then we say that the pair $\left\{L_{1}, L_{2}\right\}$ has property $C_{\varphi}$ and similarly $C_{\psi}$ is defined, $\psi_{j}$ replacing $\varphi_{j}$ in (1.8).

Theorem 1.1 The pair $\left\{L_{1}, L_{2}\right\}$, where $L_{j}:=-\frac{d^{2}}{d x^{2}}+q_{j}(x), q_{j} \in L_{1,1}\left(\mathbb{R}_{+}\right), j=$ 1,2 , has properties $C_{+}, C_{\varphi}$ and $C_{\psi}$. If $q_{j} \in L_{1,1}\left(\mathbb{R}_{-}\right)$, then $\left\{L_{1}, L_{2}\right\}$ has property $C_{-}$.

Proof Proof can be found in [18]. We sketch only the idea of the proof of property $C_{+}$.

Using the known formula

$$
f_{j}(x, k)=e^{i k x}+\int_{x}^{\infty} A_{j}(x, y) e^{i k y} d y, \quad j=1,2,
$$

where $A_{j}(x, y)$ is the transformation kernel corresponding to the potential $q_{j}(x)$, $j=1,2$, see also formula (2.17) below, and substituting (1.9) into (1.6), one gets after a change of order of integration a homogeneous Volterra integral equation for $p(x)$. Thus $p(x)=0$.

The reason for taking $b<\infty$ in (1.8) is: when one uses the formula

$$
\varphi_{j}(x, k)=\frac{\sin (k x)}{k}+\int_{0}^{x} K_{j}(x, y) \frac{\sin (k y)}{k} d y, \quad j=1,2,
$$

for the solution $\varphi_{j}$ to (1.1) (with $q=q_{j}$ ) satisfying first two conditions (1.4), then the Volterra-type integral equation for $p(x)$ contains integrals over the infinite interval $(x, \infty)$. In this case the conclusion $p(x)=0$ does not hold, in general. If, however, the integrals are over a finite interval $(x, b)$, then one can conclude $p(x)=0$.

The same argument holds when one proves property $C_{\psi}$, but formula (1.10) is replaced by

$$
\psi_{j}(x, k)=\cos (k x)+\int_{0}^{x} \widetilde{K}_{j}(x, y) \cos (k y) d y, \quad j=1,2,
$$

with a different kernel $\widetilde{K_{j}}(x, y)$.

\section{Applications of property $\mathrm{C}$}

2.1 Uniqueness of the solution inverse scattering problem with the data I-function. The I-function $I(k)$ is defined by the formula

$$
I(k):=\frac{f^{\prime}(0, k)}{f(k)} .
$$

This function is equal to the Weyl function $m(k)$ which is defined as the function for which

$$
W(x, k):=\psi(x, k)+m(k) \varphi(x, k) \in L^{2}\left(\mathbb{R}_{+}\right), \quad I m k>0,
$$


where $W(x, k)$ is the Weyl solution, $W(0, k)=1, W^{\prime}(0, k)=m(k)$. Note that $W(x, k)=\frac{f(x, k)}{f(k)}$, as follows from formulas (1.3), (2.1) and from formula (2.3) which says $I(k)=m(k)$.

To prove that

$$
I(k)=m(k),
$$

one argues as follows. If $q \in L_{1,1}\left(\mathbb{R}_{+}\right)$, then there is exactly one, up to a constant factor solution to (1.1) belonging to $L^{2}\left(\mathbb{R}_{+}\right)$when $\operatorname{Im} k>0$.

Since $f(x, k)$ is such a solution, one concludes that

$$
f(x, k)=c(k)[\psi(x, k)+m(k) \varphi(x, k)], \quad c(k) \neq 0 .
$$

Therefore,

$$
I(k)=\frac{\psi^{\prime}(0, k)+m(k) \varphi^{\prime}(0, k)}{\psi(0, k)+m(k) \varphi(0, k)}=m(k),
$$

as claimed, because $\psi(0, k)=1, \varphi(0, k)=0, \psi^{\prime}(0, k)=0$ and $\varphi^{\prime}(0, k)=1$.

In sections 11 and 12 the $I$-function is studied in more detail.

The inverse problem (IP1) is:

Given $I(k)$ for all $k>0$, find $q(x)$.

Theorem 2.1 The IP1 has at most one solution.

Proof Theorem 2.1 can be proved in several ways. One way [19] is to recover the spectral function $\rho(\lambda)$ from $I(k), k=\lambda^{\frac{1}{2}}$. This is possible since $\operatorname{Im} I(k)=$ $\frac{k}{|f(k)|^{2}}, k>0$, and

$$
d \rho(\lambda)= \begin{cases}\frac{\sqrt{\lambda} d \lambda}{\pi|f(\sqrt{\lambda})|^{2}}, & \lambda>0, \\ \sum_{j=1}^{J} c_{j} \delta\left(\lambda+k_{j}^{2}\right) d \lambda, & \lambda<0, \quad k_{j}>0,\end{cases}
$$

where $-k_{j}^{2}$ are the bound states of the Dirichlet operator $L_{q}=-\frac{d^{2}}{d x^{2}}+q(x)$ in $L^{2}\left(\mathbb{R}_{+}\right), f\left(i k_{j}\right)=0, \delta(\lambda)$ is the delta-function, and

$$
c_{j}=-\frac{2 i k_{j} f^{\prime}\left(0, i k_{j}\right)}{\dot{f}\left(i k_{j}\right)}, \quad \dot{f}:=\frac{\partial f}{\partial k} .
$$

Note that $i k_{j}$ and the number $J$ in $(2.5)$ can be found as the simple poles of $I(k)$ in $\mathbb{C}_{+}$and the number of these poles, and

$$
c_{j}=-2 i k_{j} \operatorname{Res}_{k=i k_{j}} I(k)=2 k_{j} r_{j},
$$

where $i r_{j}:=\operatorname{Res}_{k=i k_{j}} I(k)$, so $r_{j}=\frac{c_{j}}{2 k_{j}}$.

It is well known that $d \rho(\lambda)$ determines $q(x)$ uniquely [14], [17]. An algorithm for recovery of $q(x)$ from $d \rho$ is known (Gelfand-Levitan). In [19] a characterization of the class of $I$-functions corresponding to potentials in $C_{l o c}^{m}\left(\mathbb{R}_{+}\right), m \geq 0$ is given.

Here we give a very simple new proof of Theorem 2.1 ( $\mathrm{cf}[18])$ :

Assume that $q_{1}$ and $q_{2}$ generate the same $I(k)$, that is, $I_{1}(k)=I_{2}(k):=I(k)$. Subtract from equation (1.1) for $f_{1}(x, k)$ this equation for $f_{2}(x, k)$ and get:

$$
L_{1} w=p f_{2}, \quad p(x):=q_{1}(x)-q_{2}(x), \quad w:=f_{1}(x, k)-f_{2}(x, k) .
$$

Multiply (2.8) by $f_{1}$ and integrate by parts:

$$
\begin{aligned}
\int_{0}^{\infty} p(x) f_{2}(x, k) f_{1}(x, k) d x & =\left.\left(w^{\prime} f_{1}-w f_{1}^{\prime}\right)\right|_{0} ^{\infty}=\left.\left(f_{1} f_{2}^{\prime}-f_{1}^{\prime} f_{2}\right)\right|_{x=0} \\
& =f_{1} f_{2}\left(I_{1}(k)-I_{2}(k)\right)=0 \quad \forall k>0,
\end{aligned}
$$


where we have used (1.3) to conclude that at infinity the boundary term vanishes. From $(2.9)$ and property $C_{+}$(Theorem 1.1) it follows that $p(x)=0$. Theorem 2.1 is proved.

2.2 Uniqueness of the solution to inverse scattering problem on the half axis. This is a classical problem [14], [17]. The scattering data are

$$
\mathcal{S}=\left\{S(k), k_{j}, s_{j}, 1 \leq j \leq J\right\}
$$

Here

$$
S(k):=\frac{f(-k)}{f(k)}
$$

is the S-matrix, $k_{j}>0$ are the same as in section 2.1 , and the norming constants $s_{j}$ are the numbers

Note that (2.6) implies

$$
s_{j}:=-\frac{2 i k_{j}}{\dot{f}\left(i k_{j}\right) f^{\prime}\left(0, i k_{j}\right)}>0
$$

$$
c_{j}=s_{j}\left[f^{\prime}\left(0, i k_{j}\right)\right]^{2} .
$$

Theorem 2.2 Data (2.10) determine $q(x) \in L_{1,1}\left(\mathbb{R}_{+}\right)$uniquely.

Proof This result is due to Marchenko [14]. We give a new short proof based on property $C([18])$. We prove that data $(2.10)$ determine $I(k)$ uniquely, and then Theorem 2.2 follows from Theorem 2.1. To determine $I(k)$ we determine $f(k)$ and $f^{\prime}(0, k)$ from data $(2.10)$.

First, let us prove that data (2.10) determine uniquely $f(k)$. Suppose there are two different functions $f(k)$ and $h(k)$ with the same data (2.10). Then

$$
\frac{f(k)}{h(k)}=\frac{f(-k)}{h(-k)}, \quad \forall k \in \mathbb{R} .
$$

The left-hand side in (2.14) is analytic in $\mathbb{C}_{+}$since $f(k)$ and $h(k)$ are, and the zeros of $h(k)$ in $\mathbb{C}_{+}$are the same as these of $f(k)$, namely $i k_{j}$, and they are simple. The right-hand side of (2.14) has similar properties in $\mathbb{C}_{-}$. Thus $\frac{f(k)}{h(k)}$ is an entire function which tends to 1 as $|k| \rightarrow \infty$, so, $\frac{f(k)}{h(k)}=1$ and $f(k)=h(k)$. The relation

$$
\lim _{|k| \rightarrow \infty, k \in \mathbb{C}_{+}} f(k)=1
$$

follows from the representation

$$
f(k)=1+\int_{0}^{\infty} A(0, y) e^{i k y} d y, \quad A(0, y) \in L_{1}\left(\mathbb{R}_{+}\right) .
$$

Various estimates for the kernel $A(x, y)$ in the formula

$$
f(x, k)=e^{i k x}+\int_{x}^{\infty} A(x, y) e^{i k y} d y
$$

are given in [14]. We mention the following:

$$
\begin{aligned}
& |A(x, y)| \leq c \sigma\left(\frac{x+y}{2}\right), \quad \sigma(x):=\int_{x}^{\infty}|q(t)| d t \\
& \left|\frac{\partial A(x, y)}{\partial x}+\frac{1}{4} q\left(\frac{x+y}{2}\right)\right| \leq c \sigma(x) \sigma\left(\frac{x+y}{2}\right)
\end{aligned}
$$




$$
\left|\frac{\partial A(x, y)}{\partial y}+\frac{1}{4} q\left(\frac{x+y}{2}\right)\right| \leq c \sigma(x) \sigma\left(\frac{x+y}{2}\right),
$$

where $c>0$ here and below stands for various estimation constants.

From (2.17) and (2.18) formula (2.16) follows.

Thus, we have proved

$$
\mathcal{S} \Rightarrow f(k)
$$

Let us prove

$$
\mathcal{S} \Rightarrow f^{\prime}(0, k)
$$

We use the Wronskian:

$$
f^{\prime}(0, k) f(-k)-f^{\prime}(0,-k) f(k)=2 i k, \quad k \in \mathbb{R} .
$$

The function $f(k)$ and therefore $f(-k)=\overline{f(k)}$, where the overbar stands for complex conjugate, we have already uniquely determined from data (2.10). Assume there are two functions $f^{\prime}(0, k)$ and $h^{\prime}(0, k)$ corresponding to the same data $(2.10)$. Let

$$
w(k):=f^{\prime}(0, k)-h^{\prime}(0, k) .
$$

Subtract $(2.23)$ with $h^{\prime}(0, \pm k)$ in place of $f^{\prime}(0, \pm k)$ from equation $(2.23)$ and get

$$
w(k) f(-k)-w(-k) f(k)=0,
$$

or

$$
\frac{w(k)}{f(k)}=\frac{w(-k)}{f(-k)} \quad \forall k \in \mathbb{R}
$$

Claim $\frac{w(k)}{f(k)}$ is analytic in $\mathbb{C}_{+}$and vanishes at infinity and $\frac{w(-k)}{f(-k)}$ is analytic in $\mathbb{C}_{-}$and vanishes at infinity.

If this claim holds, then $\frac{w(k)}{f(k)} \equiv 0, k \in \mathbb{C}$, and therefore $w(k) \equiv 0$, so $f^{\prime}(0, k)=$ $h^{\prime}(0, k)$.

To complete the proof, let us prove the claim.

From (2.17) one gets:

$$
f^{\prime}(0, k)=i k-A(0,0)+\int_{0}^{\infty} A_{x}(0, y) e^{i k y} d y
$$

Taking $k \rightarrow+\infty$ in (2.16), integrating by parts and using (2.20), one gets:

$$
f(k)=1-\frac{A(0,0)}{i k}-\frac{1}{i k} \int_{0}^{\infty} A_{y}(0, y) e^{i k y} d y .
$$

Thus

$$
A(0,0)=-\lim _{k \rightarrow \infty} i k[f(k)-1] .
$$

Since $f(k)$ is uniquely determined by data $(2.10)$, so is the constant $\mathrm{A}(0,0)$ (by formula $(2.28))$.

Therefore (2.24) and (2.28) imply:

$$
\lim _{|k| \rightarrow \infty, k \in \mathbb{C}_{+}} w(k)=0 .
$$

It remains to be checked that (2.10) implies

$$
w\left(i k_{j}\right)=0 .
$$


This follows from formula (2.12): if $f(k)$ and $s_{j}$ are the same, so are $f^{\prime}\left(0, i k_{j}\right)$, and $w\left(i k_{j}\right)=0$ as the difference of equal numbers

$$
h^{\prime}\left(0, i k_{j}\right)=f^{\prime}\left(0, i k_{j}\right)=-\frac{2 i k_{j}}{\dot{f}\left(i k_{j}\right) s_{j}} .
$$

Theorem 2.2 is proved.

In this section we have proved that the scattering data (2.10) determines the $I$ function (2.1) uniquely. The converse is also true: implicitly it follows from the fact that both sets of data (2.1) and (2.10) determine uniquely the potential and are determined by the potential uniquely. A direct proof is given in section 12 below.

2.3 Compactly supported potential is uniquely determined by the phase shift of $s$-wave. Consider the inverse scattering on half-line and assume $q(x)=0$ for $x>a>0$, where $a>0$ is an arbitrary fixed number.

The phase shift of s-wave is denoted by $\delta(k)$ and is defined by the formula

$$
f(k)=|f(k)| e^{-i \delta(k)},
$$

so the S-matrix can be written as

$$
S(k)=\frac{f(-k)}{f(k)}=e^{2 i \delta(k)} .
$$

If $q(x)$ is real-valued, then

$$
\delta(-k)=-\delta(k), \quad k \in \mathbb{R},
$$

and if $q \in L_{1,1}\left(\mathbb{R}_{+}\right)$, then

$$
\delta(\infty)=0
$$

Note that S-matrix is unitary:

$$
S(-k)=\overline{S(k)}, \quad|S(k)|=1 \text { if } k \in \mathbb{R} .
$$

Define index of $S(k)$ :

$$
\nu:=\operatorname{ind} S(k):=\frac{1}{2 \pi i} \int_{-\infty}^{\infty} d \ln S(k)=\frac{1}{2 \pi} \Delta_{\mathbb{R}} \arg S(k) .
$$

From (2.32), (2.33) and (2.34) one derives a formula for the index:

$$
\begin{aligned}
\nu & =\frac{1}{\pi} \Delta_{\mathbb{R}} \delta(k)=\frac{1}{\pi}[\delta(-0)-\delta(-\infty)+\delta(+\infty)-\delta(+0)] \\
& =-\frac{2}{\pi} \delta(+0)= \begin{cases}-2 J & \text { if } f(0) \neq 0, \\
-2 J-1 & \text { if } f(0)=0 .\end{cases}
\end{aligned}
$$

Here we have used the formula:

$$
\frac{1}{\pi} \delta(+0)=\#\left\{\text { zeros of } f(k) \text { in } \mathbb{C}_{+}\right\}+\frac{1}{2} \delta_{0},
$$

which is the argument principle. Here $\delta_{0}:=0$ if $f(0) \neq 0$ and $\delta_{0}:=1$ if $f(0)=0$.

The zero of $f(k)$ at $k=0$ is called a resonance at zero energy.

Let us prove the following result [22]:

Theorem 2.3 If $q \in L_{1,1}\left(\mathbb{R}_{+}\right)$decays faster than any exponential: $|q(x)| \leq$ $c e^{-c|x|^{\gamma}}, \gamma>1$, then the data $\{\delta(k) \forall k>0\}$ determines $q(x)$ uniquely. 
Proof Our proof is new and short. We prove that, if $q$ is compactly supported or decays faster than any exponential, e.g. $|q(x)| \leq c e^{-c|x|^{\gamma}}, \gamma>1$, then $\delta(k)$ determines uniquely $k_{j}$ and $s_{j}$, and, by Theorem $2.2, q(x)$ is uniquely determined.

We give the proof for compactly supported potentials. The proof for the potentials decaying faster than any exponentials is exactly the same. The crucial point is: under both assumptions the Jost function is an entire function of $k$.

If $q(x)$ is compactly supported, $q(x)=0$ for $x \geq a$, then $f(k)$ is an entire function of exponential type $\leq 2 a$, that is $|f(k)| \leq c e^{2 a|k|}([17$, p. 278]). Therefore $S(k)$ is meromorphic in $\mathbb{C}_{+}$(see (2.32)). Therefore the numbers $k_{j}, 1 \leq j \leq J$, can be uniquely determined as the only poles of $S(k)$ in $\mathbb{C}_{+}$. One should check that

$$
f\left(-i k_{j}\right) \neq 0 \text { if } f\left(i k_{j}\right)=0 .
$$

This follows from (2.23): if one takes $k=i k_{j}$ and uses $f\left(i k_{j}\right)=0$, then $(2.23)$ yields

$$
f^{\prime}\left(0, i k_{j}\right) f\left(-i k_{j}\right)=-2 k_{j}<0 .
$$

Thus $f\left(-i k_{j}\right) \neq 0$. Therefore $\delta(k)$ determines uniquely the numbers $k_{j}$ and $J$.

To determine $s_{j}$, note that

$$
\operatorname{Res}_{k=i k_{j}} S(k)=\frac{f\left(-i k_{j}\right)}{\dot{f}\left(i k_{j}\right)}=\frac{1}{i} s_{j},
$$

as follows from (2.12) and (2.40). Thus the data (2.10) are uniquely determined from $S(k)$ if $q$ is compactly supported, and Theorem 2.2 implies Theorem 2.3.

Corollary 2.1 If $q(r) \in L_{1,1}\left(\mathbb{R}_{+}\right)$is compactly supported, then the knowledge of $f(k)$ on an arbitrary small open subset of $\mathbb{R}_{+}$(or even on an infinite sequence $k_{n}>0, k_{n} \neq k_{m}$ if $m \neq n, k_{n} \rightarrow k$ as $\left.n \rightarrow \infty, k>0\right)$ determines $q(r)$ uniquely.

In section 4 we prove a similar result with the data $f^{\prime}(0, k)$ in place of $f(k)$.

2.4 Recovery of $q \in L_{1,1}(\mathbb{R})$ from the reflection coefficient alone. Consider the scattering problem on the full line: $u$ solves (1.1) and

$$
\begin{gathered}
u \sim t(k) e^{i k x}, \quad x \rightarrow+\infty, \\
u \sim e^{i k x}+r(k) e^{-i k x}, \quad x \rightarrow-\infty .
\end{gathered}
$$

The coefficients $t(k)$ and $r(k)$ are called the transmission and reflection coefficients (see [14] and [17]). In general $r(k)$ alone cannot determine $q(x)$ uniquely.

We assume

$$
q(x)=0 \text { for } x<0 \text {, }
$$

and give a short proof, based on property $\mathrm{C}$, of the following:

Theorem 2.4 If $q \in L_{1,1}(\mathbb{R})$ and (2.44) holds, then $r(k), \forall k>0$, determines $q(x)$ uniquely.

Proof We claim that $r(k)$ determines uniquely $I(k)$ if (2.44) holds. Thus, Theorem 2.4 follows from Theorem 2.1. To check the claim, note that $u(x, k)=$ $t(k) f(x, k)$, so

$$
I(k)=\frac{u^{\prime}(0, k)}{u(0, k)},
$$

and use (2.44), (2.43) to get $u=e^{i k x}+r(k) e^{-i k x}$ for $x<0$, so

$$
\frac{u^{\prime}(0, k)}{u(0, k)}=\frac{i k(1-r(k))}{1+r(k)} .
$$


From (2.45) and (2.46) the claim follows. Theorem 2.4 is proved.

2.5 Inverse scattering with various data. Consider scattering on the full line (1.1), (2.42)-(2.43), assume $q(x)=0$ if $x \notin[0,1]$, and take as the scattering data the function

$$
u(0, k):=u_{0}(k), \quad \forall k>0 .
$$

Theorem 2.5 Data (2.47) determine $q(x)$ uniquely.

Proof If $q=0$ for $x<0$, then $u(x, k)=e^{i k x}+r(k) e^{-i k x}$ for $x<0, u(0, k)=$ $1+r(k)$, so data $(2.47)$ determine $r(k)$ and, by Theorem $2.4, q(x)$ is uniquely determined. Theorem 2.5 is proved. Of course, this theorem is a particular case of Theorem 2.4 .

Remark 2.1 Other data can be considered, for example, $u^{\prime}(0, k):=v(k)$. Then $u^{\prime}(0, k)=i k[1-r(k)]$, and again $v(k)$ determines $r(k)$ and, by Theorem 2.4, $q(x)$ is uniquely determined.

However, if the data are given at the right end of the support of the potential, the inverse problem is more difficult. For example, if $u(1, k):=u_{1}(k)$ is given for all $k>0$, then $u_{1}(k)=t(k) e^{i k}$, so $t(k)$ is determined by the data uniquely.

The problem of determining $q(x)$ from $t(k)$ does not seem to have been studied. If $q(x) \geq 0$, then the selfadjoint operator $L_{q}=-\frac{d^{2}}{d x^{2}}+q(x)$ in $L^{2}(\mathbb{R})$ does not have bound states (negative eigenvalues).

In this case the relation $\left|r^{2}(k)\right|+\left|t^{2}(k)\right|=1$ allows one to find

$$
|r(k)|=\sqrt{1-|t(k)|^{2}}, \quad k \in \mathbb{R} .
$$

Define

$$
a(k):=\exp \left\{-\frac{1}{\pi i} \int_{-\infty}^{\infty} \frac{\ln |t(s)|}{s-k} d s\right\} .
$$

The function (2.48) has no zeros in $\mathbb{C}_{+}$if $L_{q}$ has no bound states. If $q$ is compactly supported then $r(k)$ and $t(k)$ are meromorphic in $\mathbb{C}$.

Let us note that

$$
\begin{gathered}
f(x, k):=f_{+}(x, k)=b(k) g_{-}(x, k)+a(k) g_{+}(x, k) \\
g_{-}(x, k)=c(k) f_{+}(x, k)+d(k) f_{-}(x, k) .
\end{gathered}
$$

It is known [14], [17], that

$$
\begin{gathered}
a(-k)=\overline{a(k)}, \quad b(-k)=\overline{b(k)}, \quad k \in \mathbb{R}, \\
c(k)=-b(-k), \quad d(k)=a(k), \\
a(k)=-\frac{1}{2 i k}\left[f(x, k), g_{-}(x, k)\right], \quad b(k)=\frac{1}{2 i k}\left[f_{+}(x, k), g_{+}(x, k)\right],
\end{gathered}
$$

where $[f, g]:=f g^{\prime}-f^{\prime} g$ is the Wronskian,

$$
\begin{gathered}
|a(k)|^{2}=1+|b(k)|^{2} \\
r(k)=\frac{b(k)}{a(k)}, \quad t(k)=\frac{1}{a(k)} .
\end{gathered}
$$

The function $a(k)$ is analytic in $\mathbb{C}_{+}$. One can prove [14, p.288]

$$
a(k)=1-\frac{\int_{-\infty}^{\infty} q(x) d x}{2 i k}+o\left(\frac{1}{k}\right), \quad k \rightarrow \infty,
$$


and

$$
b(k)=O\left(\frac{1}{k}\right), \quad k \rightarrow \infty .
$$

The function $r(k)$ does not allow, in general, an analytic continuation from the real axis into the complex plane. However, if $q(x)=0$ for $x<0$, then $b(k)$ admits an analytic continuation from the real axis into $\mathbb{C}_{+}$and $r(k)$ is meromorhic in $\mathbb{C}_{+}$.

If $q(x)$ is compactly supported the functions $f_{ \pm}(x, k)$ and $g_{ \pm}(x, k)$ are entire functions of $k$ of exponential type, so that $r(k)$ and $t(k)$ are meromorphic in $\mathbb{C}$. From (2.54) one can find $|b(k)|$ since $a(k)$ is found from formula (2.48) (assuming no bound states).

The conclusion is: recovery of a compactly supported potential from the transmission coefficient is an open problem.

\section{Inverse problems on a finite interval with mixed data}

Consider equation (1.1) on the interval $[0,1]$. Take some selfadjoint boundary conditions, for example:

$$
-u^{\prime \prime}+q(x) u-\lambda u=0, \quad 0 \leq x \leq 1 ; \quad u(0)=u(1)=0, \quad \lambda=k^{2} .
$$

Assume $q \in L^{1}[0,1], q=\bar{q}$. Fix $b \in(0,1)$ arbitrary. Suppose $q(x)$ is known on the interval $[b, 1]$ and the subset $\left\{\lambda_{m(n)}\right\}$ of the eigenvalues of the problem (3.1) is known, $n=1,2, \ldots$ where $m(n)$ is a sequence with the property

$$
\frac{m(n)}{n}=\frac{1}{\sigma}\left(1+\varepsilon_{n}\right), \quad \varepsilon_{n} \rightarrow 0, \quad \sigma>0 .
$$

Theorem 3.1 The data $\left\{\lambda_{m(n)}, n=1,2, \ldots ; q(x), b \leq x \leq 1\right\}$ determine uniquely $q(x), 0 \leq x \leq b$, if $\sigma>2 b$. If $\sigma=2 b$ and $\sum_{n=1}^{\infty}\left|\varepsilon_{n}\right|<\infty$, then the above data determine $q(x), 0 \leq x \leq b$, uniquely.

Proof First, assume $\sigma>2 b$. If there are $q_{1}$ and $q_{2}$ which produce the same data, then as above, one gets

$G(\lambda):=g(k):=\int_{0}^{b} p(x) \varphi_{1}(x, k) \varphi_{2}(x, k) d x=\left.\left(\varphi_{1} w^{\prime}-\varphi_{1}^{\prime} w\right)\right|_{0} ^{1}=\left.\left(\varphi_{1} w^{\prime}-\varphi_{1}^{\prime} w\right)\right|_{x=1}$

where $w:=\varphi_{1}-\varphi_{2}, p:=q_{1}-q_{2}, k=\sqrt{\lambda}$. Thus

$$
g(k)=0 \text { at } k= \pm \sqrt{\lambda_{m(n)}}:= \pm k_{n} .
$$

The function $G(\lambda)$ is an entire function of $\lambda$ of order $\frac{1}{2}$ (see formula (1.10) with $k=\sqrt{\lambda}$ ), and is an entire even function of $k$ of exponential type $\leq 2 b$. One has

$$
|g(k)| \leq c \frac{e^{2 b|\operatorname{Im} k|}}{1+|k|^{2}} .
$$

The indicator of $g$ is defined by the formula

$$
h(\theta):=h_{g}(\theta):=\varlimsup_{r \rightarrow \infty} \frac{\ln \left|g\left(r e^{i \theta}\right)\right|}{r},
$$

where $k=r e^{i \theta}$. Since $|\operatorname{Im} k|=r|\sin \theta|$, one gets from (3.5) and (3.6) the following estimate

$$
h(\theta) \leq 2 b|\sin \theta| .
$$


It is known [10, formula (4.16)] that for any entire function $g(k) \not \equiv 0$ of exponential type one has:

$$
\lim _{r \rightarrow \infty} \frac{n(r)}{r} \leq \frac{1}{2 \pi} \int_{0}^{2 \pi} h_{g}(\theta) d \theta
$$

where $n(r)$ is the number of zeros of $g(k)$ in the disk $|k| \leq r$. From (3.7) one gets

$$
\frac{1}{2 \pi} \int_{0}^{2 \pi} h_{g}(\theta) d \theta \leq \frac{2 b}{2 \pi} \int_{0}^{2 \pi}|\sin \theta| d \theta=\frac{4 b}{\pi}
$$

From (3.2) and the known asymptotics of the Dirichlet eigenvalues:

$$
\lambda_{n}=(\pi n)^{2}+c+o(1), \quad n \rightarrow \infty, \quad c=\text { const },
$$

one gets for the number of zeros the estimate

$$
n(r) \geq 2 \sum_{\frac{n \pi}{\sigma}\left[1+0\left(\frac{1}{n^{2}}\right)\right]<r} 1=2 \frac{\sigma r}{\pi}[1+o(1)], \quad r \rightarrow \infty .
$$

From (3.8), (3.9) and (3.11) it follows that

$$
\sigma \leq 2 b
$$

Therefore, if $\sigma>2 b$, then $g(k) \equiv 0$. If $g(k) \equiv 0$ then, by property $C_{\varphi}$ (Theorem 1.1), $p(x)=0$. Theorem 3.1 is proved in the case $\sigma>2 b$.

Assume now that $\sigma=2 b$ and

$$
\sum_{n=1}^{\infty}\left|\varepsilon_{n}\right|<\infty
$$

We claim that if an entire function $G(\lambda)$ in (3.3) of order $\frac{1}{2}$ vanishes at the points

$$
\lambda_{n}=\frac{n^{2} \pi^{2}}{\sigma^{2}}\left(1+\varepsilon_{n}\right),
$$

and (3.13) holds, then $G(\lambda) \equiv 0$. If this is proved, then Theorem 3.1 is proved as above.

Let us prove the claim. Define

$$
\Phi(\lambda):=\prod_{n=1}^{\infty}\left(1-\frac{\lambda}{\lambda_{n}}\right)
$$

and recall that

$$
\Phi_{0}(\lambda):=\frac{\sin (\sigma \sqrt{\lambda})}{\sigma \sqrt{\lambda}}=\prod_{n=1}^{\infty}\left(1-\frac{\lambda}{\mu_{n}}\right), \quad \mu_{n}:=\frac{n^{2} \pi^{2}}{\sigma^{2}} .
$$

Since $G\left(\lambda_{n}\right)=0$, the function

$$
w(\lambda):=\frac{G(\lambda)}{\Phi(\lambda)}
$$

is entire, of order $\leq \frac{1}{2}$. Let us use a Phragmen-Lindelöf lemma.

Lemma 3.1 [10, Theorem 1.22] If an entire function $w(\lambda)$ of order $<1$ has the property $\sup _{-\infty<y<\infty}|w(i y)| \leq c$, then $w(\lambda) \equiv c$. If, in addition $w(i y) \rightarrow 0$ as $y \rightarrow+\infty$, then $w(\lambda) \equiv 0$. 
We use this lemma to prove that $w(\lambda) \equiv 0$. If this is proved then $G(\lambda) \equiv 0$ and Theorem 3.1 is proved.

The function $w(\lambda)$ is entire of order $\frac{1}{2}<1$.

Let us check that

and that

$$
\sup _{-\infty<y<\infty}|w(i y)|<\infty
$$

$$
|w(i y)| \rightarrow 0 \text { as } y \rightarrow+\infty .
$$

One has, using (3.5), (3.15), (3.16) and taking into account that $\sigma=2 b$ :

$$
\begin{aligned}
|w(i y)| & =\left|\frac{G(i y)}{\Phi(i y)} \frac{\Phi_{0}(i y)}{\Phi_{0}(i y)}\right| \leq \frac{e^{2 b|\operatorname{Im} \sqrt{i y}|}}{(1+|y|)}\left(\frac{e^{\sigma|\operatorname{Im} \sqrt{i y}|}}{1+|y|^{\frac{1}{2}}}\right)^{-1}\left(\prod_{h=1}^{\infty} \frac{1+\frac{y^{2}}{\mu_{n}^{2}}}{1+\frac{y^{2}}{\lambda_{n}^{2}}}\right)^{\frac{1}{2}} \\
& \leq \frac{c}{1+|y|^{\frac{1}{2}}}\left(\prod_{\left\{n: \mu_{n} \leq \lambda_{n}\right\}} \frac{\lambda_{n}^{2}}{\mu_{n}^{2}}\right)^{\frac{1}{2}} \leq \frac{c}{1+|y|^{\frac{1}{2}}} \prod_{\left\{n: \mu_{n} \leq \lambda_{n}\right\}}\left(1+\left|\varepsilon_{n}\right|\right) \leq \frac{c_{1}}{1+|y|^{\frac{1}{2}}} .
\end{aligned}
$$

Here we have used elementary inequalities:

$$
\frac{1+a}{1+d} \leq \frac{a}{d} \quad \text { if } \quad a \geq d>0 ; \quad \frac{1+a}{1+d} \leq 1 \quad \text { if } \quad 0 \leq a \leq d
$$

with $a:=\frac{y^{2}}{\mu_{n}^{2}}, d:=\frac{y^{2}}{\lambda_{n}^{2}}$, and the assumption (3.13).

We also used the relation:

$$
\left|\frac{\sin (\sigma \sqrt{i y})}{\sigma \sqrt{i y}}\right| \sim \frac{e^{\sigma|\operatorname{Im} \sqrt{i y}|}}{2 \sigma|\sqrt{i y}|} \quad \text { as } \quad y \rightarrow+\infty .
$$

Estimate (3.20) implies (3.18) and (3.19). An estimate similar to (3.20) has been used in the literature (see e.g. [4]).

Theorem 3.1 is proved.

Remark 3.1 Theorem 3.1 yields several results obtained in [4], and an earlier result of Hochstadt-Lieberman which says that the knowledge of all the Dirichlet eigenvalues and the knowledge of $q(x)$ on $\left[\frac{1}{2}, 1\right]$ determine uniquely $q(x)$ on $\left[0, \frac{1}{2}\right]$.

In this case $b=\frac{1}{2}, \sigma=1$.

One can also obtain (from Theorem 3.1) the classical result of Borg [2] and its generalization due to Marchenko [14]:

Two spectra (with the same boundary conditions on one of the ends of the interval and two different boundary conditions on the other end) determine $q(x)$ and the boundary conditions uniquely.

\section{Property $\mathrm{C}$ and inverse problems for some PDE}

4.1 Consider the problem

$$
\begin{gathered}
u_{t}=u_{x x}-q(x) u, \quad 0 \leq x \leq 1, \quad t>0, \\
u(x, 0)=0, \\
u(0, t)=0, \quad u(1, t)=a(t) .
\end{gathered}
$$

Assume the $a(t) \not \equiv 0$ is compactly supported, $a(t) \in L^{1}(0, \infty), q(x) \in L^{1}[0,1]$, problem (4.1) - (4.3) is solvable, and one can measure the data

$$
u^{\prime}(1, t):=u_{x}(1, t):=b(t) \quad \forall t>0 .
$$


The inverse problem (IP1) is:

Given $\{a(t), b(t), \forall t>0\}$ find $q(x)$.

Theorem 4.1 IP1 has at most one solution.

Proof Laplace-transform (4.1) - (4.3) to get

$$
\begin{gathered}
v^{\prime \prime}-\lambda v-q(x) v=0 \quad 0 \leq x \leq 1, \quad v:=\int_{0}^{\infty} e^{-\lambda t} u(x, t) d t \\
v(0, \lambda)=0, \quad v(1, \lambda)=A(\lambda):=\int_{0}^{\infty} e^{-\lambda t} a(t) d t \\
v^{\prime}(1, \lambda)=B(\lambda):=\int_{0}^{\infty} b(t) e^{-\lambda t} d t
\end{gathered}
$$

Assume that there are $q_{1}(x)$ and $q_{2}(x)$ which generate the same data $\{A(\lambda), B(\lambda)$, $\forall \lambda>0\}$. Let $p(x):=q_{1}(x)-q_{2}(x), w:=v_{1}-v_{2}$. Subtract from equation (4.5) with $v=v_{1}, q=q_{1}$, similar equations with $v=v_{2}, q=q_{2}$, and get

$$
\begin{gathered}
\ell_{1} w:=w^{\prime \prime}-\lambda w-q_{1} w=p v_{2}, \\
w(0, \lambda)=0, \quad w(1, \lambda)=w^{\prime}(1, \lambda)=0 .
\end{gathered}
$$

Multiply (4.8) by $\varphi_{1}(x, \lambda)$, where $\ell_{1} \varphi_{1}=0, \varphi_{1}(0, \lambda)=0, \varphi_{1}^{\prime}(0, \lambda)=1$, integrate over $[0,1]$ and then by parts on the left-hand side, using (4.9). The result is:

$$
\int_{0}^{1} p(x) v_{2}(x, \lambda) \varphi_{1}(x, \lambda) d x=0 \quad \forall \lambda>0 .
$$

Note that $\varphi_{1}(x, \lambda)$ is an entire function of $\lambda$.

Since $a(t) \not \equiv 0$ and is compactly supported, the function $A(\lambda)$ is an entire function of $\lambda$, so it has a discrete set of zeros. Therefore $v_{2}(x, \lambda)=c(\lambda) \varphi_{2}(x, \lambda)$ where $c(\lambda) \neq 0$ for almost all $\lambda \in \mathbb{R}_{+}$.

Property $C_{\varphi}$ (Theorem 1.1) and (4.10) imply $p(x)=0$. Theorem 4.1 is proved.

Remark 4.1 One can consider different selfadjoint homogeneous boundary conditions at $x=0$, for example, $u^{\prime}(0, t)=0$ or $u^{\prime}(0, t)-h_{0} u(0, t)=0, h_{0}=$ const $>0$.

A different method of proof of a result similar to Theorem 4.1 can be found in [18] and in [5]. In [5] some extra assumptions are imposed on $q(x)$ and $a(t)$.

4.2 Consider the problem:

$$
\begin{gathered}
u_{t t}=u_{x x}-q(x) u, \quad x \geq 0, \quad t \geq 0, \\
u=u_{t}=0 \text { at } t=0, \\
u(0, t)=\delta(t),
\end{gathered}
$$

where $\delta(t)$ is the delta-function. Assume that

$$
q(x)=0 \quad \text { if } \quad x>1, \quad q=\bar{q}, \quad q \in L^{1}[0,1] .
$$

Suppose the data

$$
u(1, t):=a(t)
$$

are given for all $t>0$.

The inverse problem (IP2) is:

Given $a(t) \forall t>0$, find $q(x)$. 
Theorem 4.2 The IP2 has at most one solution.

Proof Fourier-transform (4.11)-(4.13), (4.15) to get

$$
\begin{array}{r}
v^{\prime \prime}+k^{2} v-q(x) v=0, \quad x \geq 0, \\
v(0, k)=1, \\
v(1, k)=A(k):=\int_{0}^{\infty} a(t) e^{i k t} d t,
\end{array}
$$

where

$$
v(x, k)=\int_{0}^{\infty} u(x, t) e^{i k t} d t .
$$

It follows from (4.19) and (4.16) that

$$
v(x, k)=c(k) f(x, k),
$$

where $f(x, k)$ is the Jost solution to (4.16). From (4.20) and (4.17) one gets

$$
v(x, k)=\frac{f(x, k)}{f(k)},
$$

where $f(k)=f(0, k)$. From (4.21) and (4.18) one obtains

$$
f(k)=\frac{f(1, k)}{A(k)} .
$$

From (4.14) and (4.16) one concludes

$$
f(x, k)=e^{i k x} \text { for } x \geq 1 .
$$

From (4.23) and (4.22) one gets

$$
f(k)=\frac{e^{i k}}{A(k)} .
$$

Thus $f(k)$ is known for all $k>0$.

Since $q(x)$ is compactly supported, the data $\{f(k), \forall k=0\}$ determine $q(x)$ uniquely by Theorem 2.3. Theorem 4.2 is proved.

Remark 4.2 One can consider other data at $x=1$, for example, the data $u_{x}(1, t)$ or $u_{x}(1, t)+h u(1, t)$. The argument remains essentially the same.

However, the argument needs a modification if (4.13) is replaced by another condition, for example, $u_{x}(0, t)=\delta(t)$. In this case $v(x, k)=\frac{f(x, k)}{f^{\prime}(0, k)}$, and in place of $f(k)$ one obtains $f^{\prime}(0, k) \forall k>0$ from the data (4.18).

The problem of finding a compactly supported $q(x)$ from the data $\left\{f^{\prime}(0, k) \forall k>\right.$ $0\}$ was not studied, to our knowledge. We state the following:

Claim The data $f^{\prime}(0, k)$ known on an arbitrary small open subset of $(0, \infty)$ or even on an infinite sequence of distinct positive numbers $k_{n}$ which has a limit point $k>0$, determines a compactly supported $q(r) \in L^{1}\left(\mathbb{R}_{+}\right)$uniquely.

Our approach to this problem is based on formula $(2.23)$. If $f^{\prime}(0, k)$ is known for all $k>0$, then $f^{\prime}(0,-k)=\overline{f^{\prime}(0, k)}$ is known for all $k>0$, and $(2.23)$ can be considered as the Riemann problem for finding $f(k)$ and $f(-k)$ from $(2.23)$ with the coefficients $f^{\prime}(0, k)$ and $f^{\prime}(0,-k)$ known. If $q(x) \in L_{1}\left(\mathbb{R}_{+}\right)$is compactly supported then $f^{\prime}(0, k)$ is an entire function of $k$. Thus the data determine $f^{\prime}(0, k)$, for all $k>0$. 
We want to prove that $(2.23)$ defines $f(k)$ uniquely if $f^{\prime}(0, k)$ is known for all $k>0$. Assume the contrary. Let $f(k)$ and $h(k)$ be two solutions to (2.23), and $w:=f-h, w(k) \rightarrow 0$ as $|k| \rightarrow \infty, k \in \mathbb{C}_{+}$. Then (2.23) implies

$$
\frac{w(k)}{f^{\prime}(0, k)}=\frac{w(-k)}{f^{\prime}(0,-k)} \quad \forall k \in \mathbb{R} .
$$

The function $f^{\prime}(0, k)$ has at most finitely many zeros in $\mathbb{C}_{+}$. All these zeros are at the points $i \kappa_{j}, 1 \leq j \leq J_{1}$, where $-\kappa_{j}^{2}$ are the negative eigenvalues of the Neumann operator $L_{q}:=-\frac{d^{2}}{d x^{2}}+q(x)$ in $L^{2}\left(\mathbb{R}_{+}\right), u^{\prime}(0)=0$. Also $f^{\prime}(0,0)$ may vanish.

From (2.23) one concludes that $w\left(i \kappa_{j}\right)=0$ if $f^{\prime}\left(0, i \kappa_{j}\right)=0$. Indeed, one has $w\left(i \kappa_{j}\right) f^{\prime}\left(0,-i \kappa_{j}\right)=w\left(-i \kappa_{j}\right) f^{\prime}\left(0, i \kappa_{j}\right)$. If $f^{\prime}\left(0, i \kappa_{j}\right)=0$ then $f^{\prime}\left(0,-i \kappa_{j}\right) \neq 0$ as follows from $(2.23)$. Therefore $w\left(i \kappa_{j}\right)=0$ as claimed, and the function $\frac{w(k)}{f^{\prime}(0, k)}$ is analytic in $\mathbb{C}_{+}$and vanishes at infinity in $\mathbb{C}_{+}$. Similary, the right-hand side of (4.25) is analytic in $\mathbb{C}_{-}$and vanishes at infinity in $\mathbb{C}_{-}$. Thus, by analytic continuation, $\frac{w(k)}{f^{\prime}(0, k)}$ is an entire function which vanishes at infinity and therefore vanishes identically. Therefore $w(k) \equiv 0$ and $f(k)=h(k)$. Thus, the data $\left\{f^{\prime}(0, k), \forall k>0\right\}$ determines uniquely $\{f(k), \forall k>0\}$.

Since $q(x)$ is compactly supported, Theorem 2.3 implies that $q(x)$ is uniquely determined by the above data. The claim is proved.

\section{Invertibility of the steps in the inversion provedures in the inverse scattering and spectral problems}

5.1 Inverse spectral problem. Consider a selfadjoint operator $L_{q}$ in $L^{2}\left(\mathbb{R}_{+}\right)$ generated by the differential expression $L_{q}=-\frac{d^{2}}{d x^{2}}+q(x), q(x) \in L_{l o c}^{1}\left(\mathbb{R}_{+}\right), q(x)=$ $\overline{q(x)}$, and a selfadjoint boundary condition at $x=0$, for example, $u(0)=0$. Other selfadjoint conditions can be assumed. For instance: $u^{\prime}(0)=h_{0} u(0), h_{0}=$ const $>$ 0 .

We assume that $q(x)$ is such that the equation (1.1) with $\operatorname{Im} \lambda>0, \lambda:=k^{2}$, has exactly one solution which belongs to $L^{2}\left(\mathbb{R}_{+}\right)$(the limit-point case at infinity).

In this case there is exactly one spectral function $\rho(\lambda)$ of the selfadjoint operator $L_{q}$. Denote

$$
\varphi_{0}(x, \lambda):=\frac{\sin (\sqrt{\lambda} x)}{\sqrt{\lambda}} .
$$

Let $h(x) \in L_{0}^{2}\left(\mathbb{R}_{+}\right)$, where $L_{0}^{2}\left(\mathbb{R}_{+}\right)$denotes the set of $L^{2}\left(\mathbb{R}_{+}\right)$functions vanishing outside a compact interval (this interval depends on $h(x)$ ). Denote

$$
H(\lambda):=\int_{0}^{\infty} h(x) \varphi_{0}(x, \lambda) d x .
$$

Assume that for every $h \in L_{0}^{2}\left(\mathbb{R}_{+}\right)$one has:

$$
\int_{-\infty}^{\infty} H^{2}(\lambda) d \rho(\lambda)=0 \Rightarrow h(x)=0 .
$$

Denote by $\mathcal{P}$ the set of nondecreasing functions $\rho(\lambda)$, of bounded variation, such that if $\rho_{1}, \rho_{2} \in \mathcal{P}, \nu:=\rho_{1}-\rho_{2}$, and

$$
\mathcal{H}:=\left\{H(\lambda): h \in C_{0}^{\infty}\left(\mathbb{R}_{+}\right)\right\}
$$


where $H(\lambda)$ is given by (5.2), then

$$
\left\{\int_{-\infty}^{\infty} H^{2}(\lambda) d \nu(\lambda)=0 \quad \forall H \in \mathcal{H}\right\} \Rightarrow \nu(\lambda)=0 .
$$

Theorem 5.1 Spectral functions of the operators $L_{q}$, in the limit- point at infinity case, belong to $\mathcal{P}$.

Proof Let $b>0$ be arbitrary, $f \in L^{2}(0, b), f=0$ if $x>b$. Suppose

$$
\int_{-\infty}^{\infty} H^{2}(\lambda) d \nu(\lambda)=0 \quad \forall H \in \mathcal{H} .
$$

Denote by $I+V$ and $I+W$ the transformation operators corresponding to potentials $q_{1}$ and $q_{2}$ which generate spectral functions $\rho_{1}$ and $\rho_{2}, \nu=\rho_{1}-\rho_{2}$. Then

$$
\varphi_{0}=(I+V) \varphi_{1}=(I+W) \varphi_{2},
$$

where $V$ and $W$ are Volterra-type operators. Condition (5.6) implies:

$$
\left\|\left(I+V^{*}\right) f\right\|=\left\|\left(I+W^{*}\right) f\right\| \quad \forall f \in L^{2}(0, b),
$$

where $V^{*}$ is the adjoint operator and the norm in $(5.8)$ is $L^{2}(0, b)$-norm. Note that

$$
V f:=\int_{0}^{x} V(x, y) f(y) d y,
$$

and

$$
V^{*} f=\int_{s}^{b} V(y, s) f(y) d y .
$$

From (5.8) it follows that

$$
I+V^{*}=U\left(I+W^{*}\right),
$$

where $U$ is a unitary operator in the Hilbert space $H=L^{2}(0, b)$.

If $U$ is unitary and $V, W$ are Volterra operators then (5.11) implies $V=W$.

This is proved in Lemma 5.1 below. If $V=W$ then $\varphi_{1}(x, \lambda)=\varphi_{2}(x, \lambda)$, therefore $q_{1}=q_{2}$ and $\rho_{1}(\lambda)=\rho_{2}(\lambda)$. Here we have used the assumption about $L_{q}$ being in the limit-point at infinity case: this assumption implies that the spectral function is uniquely determined by the potential (in the limit-circle case at infinity there are many spectral functions corresponding to the given potential). Thus if $q_{1}=q_{2}$, then $\rho_{1}(\lambda)=\rho_{2}(\lambda)$. Theorem 5.1 is proved.

Lemma 5.1 Assume that $U$ is unitary and $V, W$ are Volterra operators in $H=L^{2}(0, b)$. Then (5.11) implies $V=W$.

Proof From (5.11) one gets $I+V=(I+W) U^{*}$ and, using $U^{*} U=I$, one gets

$$
(I+V)\left(I+V^{*}\right)=(I+W)\left(I+W^{*}\right) .
$$

Denote

$$
(I+V)^{-1}=I+V_{1}, \quad(I+W)^{-1}=I+W_{1},
$$

where $V_{1}, W_{1}$ are Volterra operators. From (5.12) one gets:

$$
\left(I+V^{*}\right)\left(I+W_{1}^{*}\right)=\left(I+V_{1}\right)(I+W),
$$

or

$$
V^{*}+W_{1}^{*}+V^{*} W_{1}^{*}=V_{1}+W+V_{1} W .
$$


Since the left-hand side in (5.15) is a Volterra operator of the type (5.10) while the right-hand side is a Volterra operator of the type (5.9), they can be equal only if each equals zero:

$$
V^{*}+W_{1}^{*}+V^{*} W_{1}^{*}=0
$$

and

$$
V_{1}+W+V_{1} W=0
$$

From (5.17) one gets

$$
V_{1}(I+W)=-W,
$$

or $\left[(I+V)^{-1}-I\right](I+W)=-W$. Thus $(I+V)^{-1}(I+W)=I$ and $V=W$ as claimed. Lemma 5.1 is proved.

The inverse spectral problem consists of finding $q(x)$ given $\rho(\lambda)$. The uniqueness of the solution to this problem was proved by Marchenko [14] while the reconstruction algorithm was given by Gelfand and Levitan [11] (see also [17]).

Let us prove first the uniqueness theorem of Marchenko following [20]. In this theorem there is no need to assume that $L_{q}$ is in the limit-point at infinity case: if it is not, the spectral function determines the potential uniquely also, but the potential does not determine the spectral function uniquely.

Theorem 5.2 The spectral function determines $q(x)$ uniquely.

Proof If $q_{1}$ and $q_{2}$ have the same spectral function $\rho(\lambda)$ then

$$
\|f\|^{2}=\int_{-\infty}^{\infty}\left|F_{1}(\lambda)\right|^{2} d \rho(\lambda)=\int_{-\infty}^{\infty}\left|F_{2}(\lambda)\right|^{2} d \rho(\lambda)=\|g\|^{2}
$$

for any $f \in L^{2}(0, b), b<\infty$, where

$$
F_{j}(\lambda):=\int_{0}^{b} f(x) \varphi_{j}(x, \lambda) d x, \quad j=1,2,
$$

the function $\varphi_{j}(x, \lambda)$ solves equation (1.1) with $q=q_{j}$, and $k^{2}=\lambda$, satisfies first two conditions (1.4), and

$$
g:=\left(I+K^{*}\right) f
$$

where $I+K$ is the transformation operator:

$$
\varphi_{2}=(I+K) \varphi_{1}=\varphi_{1}+\int_{0}^{x} K(x, y) \varphi_{1}(y, \lambda) d y
$$

Note that

$$
F_{2}(\lambda)=\int_{0}^{b} f(x)(I+K) \varphi_{1} d x=\int_{0}^{b} g(x) \varphi_{1}(x, \lambda) d x .
$$

From (5.19) it follows that

$$
\|f\|=\left\|\left(I+K^{*}\right) f\right\| \quad \forall f \in L^{2}(0, b):=H .
$$

Since Range $\left(I+K^{*}\right)=H$, equation (5.24) implies that $I+K$ is unitary (an isometry whose range is the whole space $H)$. Thus

$$
I+K=\left(I+K^{*}\right)^{-1}=I+T^{*}
$$

where $T^{*}$ is a Volterra operator of the type (5.10).

Therefore $K=T^{*}$ and this implies $K=T^{*}=0$. Therefore $\varphi_{1}=\varphi_{2}$ and $q_{1}=q_{2}$. Theorem 5.2 is proved. 
Let $d \rho_{j}(\lambda), j=1,2$, be the spectral functions corresponding to the operators $L_{q_{j}}$. Assume that $d \rho_{1}(\lambda)=c d \rho_{2}(\lambda)$, where $c>0$ is a constant. The above argument can be used with a minor change to prove that this assumption implies: $c=1$ and $q_{1}=q_{2}$. Indeed, the above assumption implies unitarity of the operator $\sqrt{c}(I+K)$. Therefore $c(I+K)=I+T^{*}$. Thus $c=1$ and $K=T^{*}=0$, as in the proof of Theorem 5.2. Here we have used a simple claim:

If $b I+Q=0$, where $b=$ const and $Q$ is a linear compact operator in $H$, then $b=0$ and $Q=0$.

To prove this claim, take an arbitrary orthonormal basis $\left\{u_{n}\right\}$ of the Hilbert space $H$. Then $\left\|Q u_{n}\right\| \rightarrow 0$ as $n \rightarrow \infty$ since $Q$ is compact. Note that $\left\|u_{n}\right\|=1$, so $b=\left\|b u_{n}\right\|=\left\|Q u_{n}\right\| \rightarrow 0$ as $n \rightarrow \infty$. Therefore $b=0$ and consequently $Q=0$. The claim is proved.

The Gelfand-Levitan (GL) reconstruction procedure is:

$$
\rho(\lambda) \Rightarrow L(x, y) \Rightarrow K(x, y) \Rightarrow q(x) \text {. }
$$

Here

$$
\begin{gathered}
L(x, y):=\int_{-\infty}^{\infty} \varphi_{0}(x, \lambda) \varphi_{0}(y, \lambda) d \sigma(\lambda), \quad d \sigma=d \rho-d \rho_{0}, \\
d \rho_{0}= \begin{cases}\frac{\sqrt{\lambda} d \lambda}{\pi}, & \lambda>0, \\
0 & \lambda<0 .\end{cases}
\end{gathered}
$$

Compare (5.28) and (2.5) and conclude that $\rho_{0}$ is the spectral function corresponding to the Dirichlet operator $\ell_{q}=-\frac{d^{2}}{d x^{2}}+q(x)$ in $L^{2}\left(\mathbb{R}_{+}\right)$with $q(x)=0$.

The function $K(x, y)$ defines the transformation operator (cf. (1.10))

$$
\varphi(x, \lambda)=\varphi_{0}(x, \lambda)+\int_{0}^{x} K(x, y) \varphi_{0}(y, \lambda) d y, \quad \varphi_{0}:=\frac{\sin (x \sqrt{\lambda})}{\sqrt{\lambda}}
$$

where $\varphi$ solves (1.1) with $k^{2}=\lambda$ and satisifies first two conditions (1.4).

One can prove (see [11], [17]), that $K$ and $L$ are related by the Gelfand-Levitan equation:

$$
K(x, y)+L(x, y)+\int_{0}^{x} K(x, t) L(t, y) d t=0, \quad 0 \leq y \leq x .
$$

Let us assume that the data $\rho(\lambda)$ generate the kernel $L(x, y)$ (by formula $(5.27)$ ) such that equation (5.30) is a Fredholm-type equation in $L^{2}(0, x)$ for $K(x, y)$ for any fixed $x>0$.

Then, one can prove that assumption (5.3) implies the unique solvability of the equation $(5.30)$ for $K(x, y)$ in the space $L^{2}(0, x)$.

Indeed, the homogeneous equation (5.30)

$$
h(y)+\int_{0}^{x} L(t, y) h(t) d t=0, \quad 0 \leq y \leq x,
$$

implies $h=0$ if (5.3) holds. To see this, multiply (5.21) by $h$ and integrate over $(0, x)$ (assuming without loss of generaity that $h=\bar{h}$, since the kernel $L(t, y)$ is real-valued). The result is:

$$
0=\|h\|^{2}+\int_{-\infty}^{\infty}|H(\lambda)|^{2} d \rho(\lambda)-\int_{-\infty}^{\infty}|H(\lambda)|^{2} d \rho_{0}(\lambda)
$$


or, by Parseval's equality,

$$
\int_{-\infty}^{\infty}|H(\lambda)|^{2} d \rho(\lambda)=0
$$

From (5.32) and (5.3) it follows that $h(y)=0$. Therefore, by Fredholm's alternative, equation (5.30) is uniquely solvable.

If $K(x, y)$ is its solution, then

$$
q(x)=2 \frac{d K(x, x)}{d x} .
$$

If $K(x, x)$ is a $C^{m+1}$ function then $q(x)$ is a $C^{m}$-function.

One has to prove that potential (5.22) generate the spectral function $\rho(\lambda)$ with which we started the inversion procedure (5.26).

We want to prove more, namely the following:

Theorem 5.3 Each step in the diagram (5.26) is invertible, so

$$
\rho \Leftrightarrow L \Leftrightarrow K \Leftrightarrow q .
$$

Proof 1) Step $\rho \Rightarrow L$ is done by formula (5.27).

Let us prove $L \Rightarrow \rho$. Assume there are $\rho_{1}$ and $\rho_{2}$ corresponding to the same $L(x, y)$. Then

$$
0=\int_{-\infty}^{\infty} \varphi_{0}(x, \lambda) \varphi_{0}(y, \lambda) d \nu(\lambda) \quad \forall x, y \in \mathbb{R}, \nu:=\rho_{1}-\rho_{2} .
$$

Therefore

$$
0=\int_{-\infty}^{\infty} H^{2}(\lambda) d \nu \quad \forall h \in C_{0}^{\infty}\left(\mathbb{R}_{+}\right)
$$

By Theorem 5.1 relation (5.36) implies $\nu(\lambda)=0$, so $\rho_{1}=\rho_{2}$.

2) Step $L \Rightarrow K$ is done by solving equation (5.30) for $K(x, y)$. The unique solvability of this equation for $K(x, y)$ has been proved below formula (5.30).

Let up prove $K \Rightarrow L$. From (5.27) one gets

$$
L(x, y)=\frac{L(x+y)-L(x-y)}{2}, \quad L(x):=\int_{-\infty}^{\infty} \frac{1-\cos (x \sqrt{\lambda})}{\lambda} d \sigma(\lambda) .
$$

Let $y=x$ in (5.30) and write (5.30) as

$$
L(2 x)+\int_{0}^{x} K(x, t)[L(x+t)-L(t-x)] d t=-2 K(x, x), \quad x \geq 0 .
$$

Note that $L(-x)=L(x)$. Thus (5.38) can be written as:

$$
L(2 x)+\int_{x}^{2 x} K(x, s-x) L(s) d s-\int_{0}^{x} K(x, x-s) L(s) d s=-2 K(x, x), \quad x>0 .
$$

This is a Volterra integral equation for $L(s)$. Since it is uniquely solvable, $L(s)$ is uniquely recovered from $K(x, y)$ and the step $K \Rightarrow L$ is done.

3) Step $K \Rightarrow q$ is done by equation (5.33).

The converse step $q \Rightarrow K$ is done by solving the Goursat problem:

$$
\begin{gathered}
K_{x x}-q(x) K=K_{y y} \quad 0 \leq y \leq x, \\
K(x, x)=\frac{1}{2} \int_{0}^{x} q(t) d t, \quad K(x, 0)=0 .
\end{gathered}
$$


One can prove that any twice differentiable solution to (5.30) solves (5.40)-(5.41) with $q(x)$ given by (5.33). The Goursat problem (5.40)-(5.41) is known to have a unique solution. Problem (5.40)-(5.41) is equivalent to a Volterra equation ([14], $[17])$.

Namely if $\xi=x+y, \eta=x-y, K(x, y):=B(\xi, \eta)$, then (5.40)-(5.41) take the form

$$
B_{\xi \eta}=\frac{1}{4} q\left(\frac{\xi+\eta}{2}\right) B(\xi, \eta), \quad B(\xi, 0)=\frac{1}{2} \int_{0}^{\frac{\xi}{2}} q(t) d t, \quad B(\xi, \xi)=0 .
$$

Therefore

$$
B(\xi, \eta)=\frac{1}{4} \int_{\eta}^{\xi} q\left(\frac{t}{2}\right) d t+\frac{1}{4} \int_{\eta}^{\xi} d t \int_{0}^{\eta} q\left(\frac{t+s}{2}\right) B(t, s) d s .
$$

This Volterra equation is uniquely solvable for $B(\xi, \eta)$.

Theorem 5.3 is proved.

5.2 Inverse scattering problem on the half-line. This problem consists of finding $q(x)$ given the data (2.10). Theorem 2.2 guarantees the uniqueness of the solution of this inverse problem in the class $L_{1,1}:=L_{1,1}\left(\mathbb{R}_{+}\right)$of the potentials.

The characterization of the scattering data (2.10) is known $([14],[17])$, that is, necessary and sufficient conditions on $\mathcal{S}$ for $\mathcal{S}$ to be the scattering data corresponding to a $q(x) \in L_{1,1}$. We state the result without proof. A proof can be found in [17]. A different but equivalent version of the result is given in [14].

Theorem 5.4 For the data (2.10) to be the scattering data corresponding to a $q \in L_{1,1}$ it is necessary and sufficient that the following conditions hold:

$$
\begin{aligned}
\text { i) } & \text { ind } S(k)=-\kappa \leq 0, \quad \kappa=2 J \quad \text { or } \quad \kappa=2 J+1, \\
\text { ii) } & k_{j}>0, \quad s_{j}>0, \quad 1 \leq j \leq J, \\
\text { iii) } & \overline{S(k)}=S(-k)=S^{-1}(k), S(\infty)=1, k \in \mathbb{R}, \\
\text { iv) } & \|F(x)\|_{L^{\infty}\left(\mathbb{R}_{+}\right)}+\|F(x)\|_{L^{1}\left(\mathbb{R}_{+}\right)}+\left\|x F^{\prime}(x)\right\|_{L^{1}\left(\mathbb{R}_{+}\right)}<\infty .
\end{aligned}
$$

Here $\kappa=2 J+1$ if $f(0)=0$ and $\kappa=2 J$ if $f(0) \neq 0$, and

$$
F(x):=\frac{1}{2 \pi} \int_{-\infty}^{\infty}[1-S(k)] e^{i k x} d k+\sum_{j=1}^{J} s_{j} e^{-k_{j} x} .
$$

The following estimates are useful (see [14], p.209, [17], [36], p.569 ):

$$
\begin{gathered}
|F(2 x)+A(x, x)|<c \int_{x}^{\infty}|q(x)| d x, \quad|F(2 x)|<c \int_{x}^{\infty}|q(x)| d x, \\
\left|F^{\prime}(2 x)-\frac{q(x)}{4}\right|<c\left(\int_{x}^{\infty}|q(x)| d x\right)^{2}
\end{gathered}
$$

where $c>0$ is a constant. The Marchenko inversion procedure for finding $q(x)$ from $\mathcal{S}$ is described by the following diagram:

$$
\mathcal{S} \Rightarrow F(x) \Rightarrow A(x, y) \Rightarrow q(x) .
$$

The step $\mathcal{S} \Rightarrow F$ is done by formula (5.48).

The step $F \Rightarrow A$ is done by solving the Marchenko equation for $A(x, y)$ :

$$
A(x, y)+F(x+y)+\int_{x}^{\infty} A(x, t) F(t+y) d t=0, \quad y \geq x \geq 0 .
$$


The step $A \Rightarrow q$ is done by the formula

$$
q(x)=-2 \frac{d A(x, x)}{d x} .
$$

It is important to check that the potential $q(x)$ obtained by the scheme (5.49) generates the same data $\mathcal{S}$ with which we started the inversion scheme (5.49).

Assuming $q \in L_{1,1}$ we prove:

Theorem 5.5 Each step of the diagram (5.49) is invertible:

$$
\mathcal{S} \Leftrightarrow F \Leftrightarrow A \Leftrightarrow q \text {. }
$$

Proof 1. The step $\mathcal{S} \Rightarrow F$ is done by formula (5.48) as we have already mentioned.

The step $F \Rightarrow \mathcal{S}$ is done by finding $k_{j}, s_{j}$ and $J$ from the asymptotics of the function (5.48) as $x \rightarrow-\infty$. As a result, one finds the function

$$
F_{d}(x):=\sum_{j=1}^{J} s_{j} e^{-k_{j} x} .
$$

If $F(x)$ and $F_{d}(x)$ are known, then the function

$$
F_{S}(x):=\frac{1}{2 \pi} \int_{-\infty}^{\infty}[1-S(k)] e^{i k x} d k
$$

is known. Now the function $S(k)$ can be found by the formula

$$
S(k)=1-\int_{-\infty}^{\infty} F_{S}(x) e^{-i k x} d x .
$$

So the step $F \Rightarrow \mathcal{S}$ is done.

2. The step $F \Rightarrow A$ is done by solving equation (5.50) for $A(x, y)$. This step is discussed in the literature in detail, (see [14], [17]). If $q \in L_{1,1}$ (actually a weaker condition $\int_{0}^{\infty} x|q(x)| d x<\infty$ is used in the half-line scattering theory), then one proves that conditions i) - iv) of Theorem 5.4 are satisified, that the operator

$$
T f:=\int_{x}^{\infty} F(y+t) f(t) d t, \quad y \geq x \geq 0
$$

is compact in $L^{1}(x, \infty)$ and in $L^{2}(x, \infty)$ for any fixed $x \geq 0$, and the homogeneous version of equation $(5.50)$ :

$$
f+T f=0, \quad y \geq x \geq 0
$$

has only the trivial solution $f=0$ for every $x \geq 0$. Thus, by the Fredholm alternative, equation (5.50) is uniquely solvable in $L^{2}(x, \infty)$ and in $L^{1}(x, \infty)$. The step $F \Rightarrow A$ is done.

Consider the step $A(x, y) \Rightarrow F(x)$. Define

$$
A(y):= \begin{cases}A(0, y), & y \geq 0 \\ 0, & y<0 .\end{cases}
$$

The function $A(y)$ determines uniquely $f(k)$ by the formula:

$$
f(k)=1+\int_{0}^{\infty} A(y) e^{i k y} d y,
$$

and consequently it determines the numbers $i k_{j}$ as the only zeros of $f(k)$ in $\mathbb{C}_{+}$, the number $J$ of these zeros, and $S(k)=\frac{f(-k)}{f(k)}$. To find $F(x)$, one has to find 
$s_{j}$. Formula (2.12) allows one to calculate $s_{j}$ if $f(k)$ and $f^{\prime}\left(0, i k_{j}\right)$ are known. To find $f^{\prime}\left(0, i k_{j}\right)$, use formula $(2.26)$ and put $k=i k_{j}$ in $(2.26)$. Since $A(x, y)$ is known for $y \geq x \geq 0$, formula (2.26) allows one to calculate $f^{\prime}\left(0, i k_{j}\right)$. Thus $S(k), k_{j}, s_{j}, 1 \leq j \leq J$, are found and $F(x)$ can be calculated by formula (5.48). Step $A \Rightarrow F$ is done.

The above argument proves that the knowledge of two functions $A(0, y)$ and $A_{x}(0, y)$ for all $y \geq 0$ determines $q(x) \in L_{1,1}\left(\mathbb{R}_{+}\right)$uniquely.

Note that:

a) we have used the following scheme $A \Rightarrow \mathcal{S} \Rightarrow F$ in order to get the implication $A \Rightarrow F$,

and

b) since $\{F(x), x \geq 0\} \Rightarrow A$ and $A \Rightarrow\{F(x),-\infty<x<\infty\}$, we have proved also the following non-trivial implication $\{F(x), x \geq 0\} \Rightarrow\{F(x),-\infty<x<\infty\}$.

3. Step $A \Rightarrow q$ is done by formula (5.51). The converse step $q \Rightarrow A$ is done by solving the Goursat problem:

$$
\begin{gathered}
A_{x x}-q(x) A=A_{y y}, \quad y \geq x \geq 0, \\
A(x, x)=\frac{1}{2} \int_{x}^{\infty} q(t) d t, \quad A(x, y) \rightarrow 0 \text { as } x+y \rightarrow \infty .
\end{gathered}
$$

Problem (5.60)-(5.61) is equivalent to a Volterra integral equation for $A(x, y)$ (see $[17, \mathrm{p} .253])$.

$$
A(x, y)=\frac{1}{2} \int_{\frac{x+y}{2}}^{\infty} q(t) d t+\int_{\frac{x+y}{2}}^{\infty} d s \int_{0}^{\frac{y-x}{2}} d t q(s-t) A(s-t, s+t) .
$$

One can prove that any twice differentiable solution to (5.50) solves (5.60)(5.61) with $q(x)$ given by (5.51).

A proof can be found in [14], [11] and [17].

Theorem 5.5 is proved.

Remark 5.1 It follows from Theorem 5.5 that the potential obtained by the scheme (5.49) generates the scattering data $\mathcal{S}$ with which the inversion procedure (5.49) started.

Similarly, Theorem 5.3 shows that the potential obtained by the scheme $(5.26)$ generates the spectral function $\rho(\lambda)$ with which the inversion procedure $(5.26)$ started.

The last conclusion one can obtain only because of the assumption that $q(x)$ is such that the limit-point case at infinity is valid.

If this is not the case then there are many spectral function corresponding to a given $q(x)$, so one cannot claim that the $\rho(\lambda)$ with which we started is the (unique) spectral function which is generated by $q(x)$, it is just one of many such spectral functions.

Remark 5.2 In [20] the following new equation is derived:

$$
F(y)+A(y)+\int_{0}^{\infty} A(t) F(t+y) d t=A(-y), \quad-\infty<y<\infty,
$$

which generalizes the usual equation (5.50) at $x=0$ :

$$
F(y)+A(y)+\int_{0}^{\infty} A(t) F(t+y) d t=0, \quad y \geq 0 .
$$


Since $A(-y)=0$ for $y>0$ (see (5.58)), equation (5.64) follows from (5.63) for $y>0$. For $y=0$ equation (5.64) follows from (5.63) by taking $y \rightarrow+0$ and using (5.58).

Let us prove that equations (5.63) and (5.64) are equivalent. Note that equation (5.64) is uniquely solvable if the data (2.10) correspond to a $q \in L_{1,1}$. Since any solution of (5.63) in $L^{1}\left(\mathbb{R}_{+}\right)$solves (5.64), any solution to (5.63) equals to the unique solution $A(y)$ of (5.64) for $y>0$.

Since we are looking for the solution $A(y)$ of (5.63) such that $A=0$ for $y<0$ (see $(5.58))$ one needs only to check that (5.63) is satisfied by the unique solution of (5.64).

Lemma 5.2 Equation (5.63) and (5.64) are equivalent in $L^{1}\left(\mathbb{R}_{+}\right)$.

Proof Clearly, every $L^{1}\left(\mathbb{R}_{+}\right)$solution to (5.63) solves (5.64). Let us prove the converse. Let $A(y) \in L^{1}\left(\mathbb{R}_{+}\right)$solve (5.64). Define

$$
f(k):=1+\int_{0}^{\infty} A(y) e^{i k y} d y:=1+\widetilde{A}(k) .
$$

We wish to prove that $A(y)$ solves equation (5.63). Take the Fourier transform of (5.63) in the sense of distributions. From (5.48) one gets

$$
\widetilde{F}(\xi)=\int_{-\infty}^{\infty} F(x) e^{i \xi x} d x=1-S(-\xi)+2 \pi \sum_{j=1}^{J} s_{j} \delta\left(\xi+i k_{j}\right)
$$

and from (5.63) one obtains:

$$
\widetilde{F}(\xi)+\widetilde{A}(\xi)+\widetilde{A}(-\xi) \widetilde{F}(\xi)=\widetilde{A}(-\xi) .
$$

Add 1 to both sides of (5.67) and use (5.65) to get

$$
f(\xi)+\widetilde{F}(\xi) f(-\xi)=f(-\xi) .
$$

From (5.66) and (5.68) one gets:

$f(\xi)=\left[S(-\xi)-2 \pi \sum_{j=1}^{J} s_{j} \delta\left(\xi+i k_{j}\right)\right] f(-\xi)=f(\xi)-2 \pi f(-\xi) \sum_{j=1}^{J} s_{j} \delta\left(\xi+i k_{j}\right)=f(\xi)$.

Equation (5.69) is equivalent to (5.63) since all the transformations which led from (5.63) to (5.69) are invertible. Thus, equations (5.63) and (5.69) hold (or fail to hold) simultaneously. Equation (5.69) clearly holds because

$$
f(-\xi) \sum_{j=1}^{j} s_{j} \delta\left(\xi+i k_{j}\right)=\sum_{j=1}^{J} s_{j} f\left(i k_{j}\right)=0
$$

since $i k_{j}$ are zeros of $f(k)$.

Lemma 5.2 is proved.

The results and proofs in this section are partly new and partly are based on the results in [20] and [17]. 


\section{Inverse problem for an inhomogeneous Schrödinger equation}

Consider the problem

$$
\begin{gathered}
u^{\prime \prime}+k^{2} u-q(x) u=-\delta(x), \quad-\infty<x<\infty, \\
\frac{\partial u}{\partial|x|}-i k u \rightarrow 0 \text { as }|x| \rightarrow \infty
\end{gathered}
$$

Assume

$$
q=\bar{q}, \quad q=0 \text { for }|x|>1, \quad q \in L^{1}[-1,1] .
$$

Suppose the data

$$
\{u(-1, k), u(1, k)\}_{\forall k>0}
$$

are given.

The inverse problem (IP6) is:

Given data (6.4), find $q(x)$.

Let us also assume that

(A): The operator $L_{q}=-\frac{d^{2}}{d x^{2}}+q(x)$ in $L^{2}(\mathbb{R})$ has no negative eigenvalues.

This is so if, for example, $q(x) \geq 0$.

The results of this section are taken from [21]

Theorem 6.1 If (6.3) and (A) hold then the data (6.4) determine $q(x)$ uniquely.

Proof The solution to (6.1)-(6.2) is

$$
u=\left\{\begin{array}{l}
\frac{g(k)}{[f, g]} f(x, k), \quad x>0, \\
\frac{f(k)}{[f, g]} g(x, k), \quad x<0,
\end{array}\right.
$$

where $f=f_{+}(x, k), g=g_{-}(x, k), g(k):=g_{-}(0, k), f(k):=f(0, k),[f, g]:=$ $f g^{\prime}-f^{\prime} g=-2 i k a(k), a(k)$ is defined in (2.53), $f$ is defined in (1.3) and $g$ is defined in $(1.5)$.

The functions

$$
u(1, k)=\frac{g(k) f(1, k)}{-2 i k a(k)}, \quad u(-1, k)=\frac{f(k) g(-1, k)}{-2 i k a(k)}
$$

are the data (6.4).

Since $q=0$ when $x \notin[-1,1]$, condition (6.2) implies $f(1, k)=e^{i k}$, so one knows

$$
h_{1}(k):=\frac{g(k)}{a(k)}, \quad h_{2}(k):=\frac{f(k)}{a(k)}, \quad \forall k>0 .
$$

From (6.7), (2.49) and (2.50) one derives

$$
a(k) h_{1}(k)=-b(-k) f(k)+a(k) f(-k)=-b(-k) h_{2}(k) a(k)+h_{2}(-k) a(-k) a(k),
$$

and

$$
a(k) h_{2}(k)=b(k) a(k) h_{1}(k)+a(k) h_{1}(-k) a(-k) .
$$

From (6.8) and (6.9) one gets

$$
-b(-k) h_{2}(k)+a(-k) h_{2}(-k)=h_{1}(k),
$$

and

$$
b(k) h_{1}(k)+a(-k) h_{1}(-k)=h_{2}(k) .
$$

Eliminate $b(-k)$ from $(6.10)$ and $(6.11)$ to get

$$
a(k)=m(k) a(-k)+n(k), \quad \forall k \in \mathbb{R},
$$


where

$$
m(k):=-\frac{h_{1}(-k) h_{2}(-k)}{h_{1}(k) h_{2}(k)}, \quad n(k):=\frac{h_{1}(-k)}{h_{2}(k)}+\frac{h_{2}(-k)}{h_{1}(k)} .
$$

Problem (6.12) is a Riemann problem for the pair $\{a(k), a(-k)\}$, the function $a(k)$ is analytic in $\mathbb{C}_{+}:=\{k: k \in \mathbb{C}, \operatorname{Im} k>0\}$ and $a(-k)$ is analytic in $\mathbb{C}_{-}$. The functions $a(k)$ and $a(-k)$ tend to one as $k$ tends to infinity in $\mathbb{C}_{+}$and, respectively, in $\mathbb{C}_{-}$, see equation $(2.55)$.

The function $a(k)$ has finitely many simple zeros at the points $i k_{j}, 1 \leq j \leq J$, $k_{j}>0$, where $-k_{j}^{2}$ are the negative eigenvalues of the operator $\ell$ defined by the differential expression $\ell u=-u^{\prime \prime}+q(x) u$ in $L^{2}(\mathbb{R})$.

The zeros $i k_{j}$ are the only zeros of $a(k)$ in the upper half-plane $k$.

Define

One has

$$
\text { ind } a(k):=\frac{1}{2 \pi i} \int_{-\infty}^{\infty} d \ln a(k) .
$$

$$
\text { ind } a=J
$$

where $J$ is the number of negative eigenvalues of the operator $\ell$, and, using (6.14) and (6.15), one gets

$$
\text { ind } m(k)=-2\left[\text { ind } h_{1}(k)+\text { ind } h_{2}(k)\right]=-2[\text { ind } g(k)+\text { ind } f(k)-2 J] \text {. }
$$

Since $\ell$ has no negative eigenvalues by the assumption (A), it follows that $J=0$.

In this case ind $f(k)=$ ind $g(k)=0$ (see Lemma 1 below), so ind $m(k)=0$, and $a(k)$ is uniquely recovered from the data as the solution of (6.12) which tends to one at infinity. If $a(k)$ is found, then $b(k)$ is uniquely determined by equation (6.11) and so the reflection coefficient $r(k):=\frac{b(k)}{a(k)}$ is found. The reflection coefficient determines a compactly supported $q(x)$ uniquely by Theorem 2.4 .

If $q(x)$ is compactly supported, then the reflection coefficient $r(k):=\frac{b(k)}{a(k)}$ is meromorphic. Therefore, its values for all $k>0$ determine uniquely $r(k)$ in the whole complex $k$-plane as a meromorphic function. The poles of this function in the upper half-plane are the numbers $i k_{j}=1,2, \ldots, J$. They determine uniquely the numbers $k_{j}, 1 \leq j \leq J$, which are a part of the standard scattering data $\{r(k)$, $\left.k_{j}, s_{j}, 1 \leq j \leq J\right\}$, where $s_{j}$ are the norming constants.

Note that if $a\left(i k_{j}\right)=0$ then $b\left(i k_{j}\right) \neq 0$, otherwise equation (2.49) would imply $f\left(x, i k_{j}\right) \equiv 0$ in contradiction to $(1.3)$.

If $r(k)$ is meromorphic, then the norming constants can be calculated by the formula $s_{j}=-i \frac{b\left(i k_{j}\right)}{\dot{a}\left(i k_{j}\right)}=-i \operatorname{Res}_{k=i k_{j}} r(k)$, where the dot denotes differentiation with respect to $k$, and Res denotes the residue. So, for compactly supported potential the values of $r(k)$ for all $k>0$ determine uniquely the standard scatering data, that is, the reflection coefficient, the bound states $-k_{j}^{2}$, and the norming constants $s_{j}, 1 \leq j \leq J$. These data determine the potential uniquely.

Theorem 6.1 is proved.

Lemma 6.1 If $J=0$ then ind $f=$ ind $g=0$.

Proof We prove ind $f=0$. The proof of the equation ind $g=0$ is similar. Since ind $f(k)$ equals to the number of zeros of $f(k)$ in $\mathbb{C}_{+}$, we have to prove that $f(k)$ does not vanish in $\mathbb{C}_{+}$. If $f(z)=0, z \in \mathbb{C}_{+}$, then $z=i k, k>0$, and $-k^{2}$ is an eigenvalue of the operator $\ell$ in $L^{2}(0, \infty)$ with the boundary condition $u(0)=0$. 
From the variational principle one can find the negative eigenvalues of the operator $\ell$ in $L^{2}\left(\mathbb{R}_{+}\right)$with the Dirichlet condition at $x=0$ as consequitive minima of the quadratic functional. The minimal eigenvalue is:

$$
\inf _{\substack{\circ \\ u \in H^{1}\left(\mathbb{R}_{+}\right)}} \int_{0}^{\infty}\left[u^{\prime 2}+q(x) u^{2}\right] d x:=\kappa_{0}, \quad u \in \stackrel{\circ}{H^{1}}\left(\mathbb{R}_{+}\right), \quad\|u\|_{L^{2}\left(\mathbb{R}_{+}\right)}=1,
$$

where $\stackrel{\circ}{H}^{1}\left(\mathbb{R}_{+}\right)$is the Sobolev space of $H^{1}\left(\mathbb{R}_{+}\right)$-functions satisfying the condition $u(0)=0$.

On the other hand, if $J=0$, then

$$
0 \leq \inf _{u \in H^{1}(\mathbb{R})} \int_{-\infty}^{\infty}\left[u^{\prime 2}+q(x) u^{2}\right] d x:=\kappa_{1}, \quad u \in H^{1}(\mathbb{R}), \quad\|u\|_{L^{2}(\mathbb{R})}=1 .
$$

Since any element $u$ of $\stackrel{\circ}{H^{1}}\left(\mathbb{R}_{+}\right)$can be considered as an element of $H^{1}(\mathbb{R})$ if one extends $u$ to the whole axis by setting $u=0$ for $x<0$, it follows from the variational definitions (6.16) and (6.17) that $\kappa_{1} \leq \kappa_{0}$. Therefore, if $J=0$, then $\kappa_{1} \geq 0$ and therefore $\kappa_{0} \geq 0$. This means that the operator $\ell$ on $L^{2}\left(\mathbb{R}_{+}\right)$with the Dirichlet condition at $x=0$ has no negative eigenvalues. Therefore $f(k)$ does not have zeros in $\mathbb{C}_{+}$, if $J=0$. Thus $J=0$ implies ind $f(k)=0$.

Lemma 6.1 is proved.

The above argument shows that in general

$$
\text { ind } f \leq J \text { and } \text { ind } g \leq J \text {, }
$$

so that $(6.15)$ implies

$$
\text { ind } m(k) \geq 0 \text {. }
$$

Therefore the Riemann problem (2.17) is always solvable. It is of interest to study the case when assumption (A) does not hold.

\section{Inverse scattering problem with fixed energy data}

7.1 Three-dimensional inverse scattering problem. Property C. The scattering problem in $\mathbb{R}^{3}$ consists of finding the scattering solution $u:=u(x, \alpha, k)$ from the equation

$$
\left[\nabla^{2}+k^{2}-q(x)\right] \psi=0 \text { in } \mathbb{R}^{3}
$$

and the radiation condition at infinity:

$$
\begin{gathered}
\psi=\psi_{0}+v, \quad \psi_{0}:=e^{i k \alpha \cdot x}, \quad \alpha \in S^{2}, \\
\lim _{r \rightarrow \infty} \int_{|s|=r}\left|\frac{\partial v}{\partial|x|}-i k v\right|^{2} d s=0 .
\end{gathered}
$$

Here $k>0$ is fixed, $S^{2}$ is the unit sphere, $\alpha \in S^{2}$ is given. One can write

$$
v=A\left(\alpha^{\prime}, \alpha, k\right) \frac{e^{i k r}}{r}+o\left(\frac{1}{r}\right) \text { as } r=|x| \rightarrow \infty, \quad \frac{x}{r}=\alpha^{\prime} .
$$

The coefficient $A\left(\alpha^{\prime}, \alpha, k\right)$ is called the scattering amplitude. In principle, it can be measured. We consider its values for $\alpha^{\prime}, \alpha \in S^{2}$ and a fixed $k>0$ as the scattering data. Below we take $k=1$ without loss of generality.

Assume that

$$
q \in Q_{a}:=\left\{q: q=\bar{q}, \quad q=0 \text { for }|x|>a, \quad q \in L^{p}\left(B_{a}\right)\right\},
$$

where $a>0$ is an arbitrary large fixed number, $B_{a}=\{x:|x| \leq a\}, p>\frac{3}{2}$. 
It is known (even for much larger class of the potentials $q$ ) that problem (7.1)(7.3) has the unique solution.

Therefore the map $q \rightarrow A\left(\alpha^{\prime}, \alpha\right):=A_{q}\left(\alpha^{\prime}, \alpha\right)$ is well defined,

$$
A\left(\alpha^{\prime}, \alpha\right):=\left.A\left(\alpha^{\prime}, \alpha, k\right)\right|_{k=1}
$$

(IP7) The inverse scattering problem with fixed-energy data consists of finding $q(x) \in Q_{a}$ from the scattering data $A\left(\alpha^{\prime}, \alpha\right) \forall \alpha^{\prime}, \alpha \in S^{2}$.

Uniqueness of the solution to IP7 for $q \in Q_{a}$ (with $p=2$ ) was first announced in [27] and proved in [28] by the method, based on property $\mathrm{C}$ for pairs of differential operators. The essence of this method is briefly explained below. This method was introduced by the author [26] and applied to many inverse problems [27]-[31], [24], [18] [17].

In [17] and [25] a characterization of the fixed-energy scattering amplitudes is given.

Let $\left\{L_{1}, L_{2}\right\}$ be two linear formal differential expressions,

$$
L_{j} u=\sum_{|m|=0}^{M_{j}} a_{m j}(x) \partial^{m} u(x), \quad x \in \mathbb{R}^{n}, \quad n>1, \quad j=1,2,
$$

where

$$
\partial^{m}:=\frac{\partial^{|m|}}{\partial x_{1}^{m_{1}} \ldots \partial x_{n}^{m_{n}}}, \quad|m|=m_{1}+\cdots+m_{n}
$$

Let

$$
N_{j}:=N_{j}(D):=\left\{w: L_{j} w=0 \text { in } D \subset R^{n}\right\}
$$

where $D$ is an abitrary fixed bounded domain and the equation in (7.7) is understood in the sense of distributions.

Suppose that

$$
\int_{D} f(x) w_{1}(x) w_{2}(x) d x=0, \quad w_{j} \in N_{j}, \quad f \in L^{2}(D),
$$

where $w_{j} \in N_{j}$ run through such subsets of $N_{j}, j=1,2$, that the products $w_{1} w_{2} \in$ $L^{2}(D)$, and $f \in L^{2}(D)$ is an arbitrary fixed function.

Definition 7.1 The pair $\left\{L_{1}, L_{2}\right\}$ has property $C$ if (7.8) implies $f(x)=0$, that is, the set $\left\{w_{1}, w_{2}\right\}_{\forall w_{j} \in N_{j}, w_{1} w_{2} \in L^{2}(D)}$ is complete in $L^{2}(D)$.

In [31] a necessary and sufficient condition is found for a pair $\left\{L_{1}, L_{2}\right\}$ with constant coefficients, $a_{m j}(x)=a_{m j}=$ const, to have property C (see also [17]).

In [28] it is proved that the pair $\left\{L_{1}, L_{2}\right\}$ with $L_{j}=-\nabla^{2}+q(x), q_{j} \in Q_{a}$, has property C.

The basic idea of the proof of the uniqueness theorem for inverse scattering problem with fixed-energy data, introduced in [27], presented in detail in [28], and developed in [17], [29]-[31], is simple. Assume that there are two potentials, $q_{1}$ and $q_{2}$ in $Q_{a}$ which generate the same scattering data, that is, $A_{1}=A_{2}$, where $A_{j}:=A_{q_{j}}\left(\alpha^{\prime}, \alpha\right), j=1,2$.

We prove that $[17$, p.67]

$$
-4 \pi\left(A_{1}-A_{2}\right)=\int_{B_{a}}\left[q_{1}(x)-q_{2}(x)\right] \psi_{1}(x, \alpha) \psi_{2}\left(x,-\alpha^{\prime}\right) d x \quad \forall \alpha, \alpha^{\prime} \in S^{2},
$$

where $\psi_{j}(x, \alpha)$ is the scattering solution corresponding to $q_{j}, j=1,2$. 
If $A_{1}=A_{2}$, then (7.9) yields an orthogonality relation:

$$
\int_{D} p(x) \psi_{1}(x, \alpha) \psi_{2}(x, \beta) d x=0 \quad \forall \alpha, \beta \in S^{2}, \quad p(x):=q_{1}-q_{2} .
$$

Next we prove $[17$, p.45] that

$$
\operatorname{span}_{\alpha \in S^{2}}\{\psi(x, \alpha)\} \text { is dense in } L^{2}(D) \text { in } N_{j}(D) \cap H^{2}(D),
$$

where $H^{m}(D)$ is the Sobolev space. Thus (7.10) implies

$$
\int_{D} p(x) w_{1}(x) w_{2}(x) d x=0 \quad \forall w_{j} \in N_{j}(D) \cap H^{2}(D) .
$$

Finally, by property $\mathrm{C}$ for a pair $\left\{L_{1}, L_{2}\right\}, L_{j}=-\nabla^{2}+q_{j}, q_{j} \in Q_{a}$, one concludes from (7.11) that $p(x)=0$, i.e. $q_{1}=q_{2}$. We have obtained

Theorem 7.1 (Ramm [28], [17]) The data $A\left(\alpha^{\prime}, \alpha\right) \quad \forall \alpha^{\prime}, \alpha \in S^{2}$ determine $q \in Q_{a}$ uniquely.

This is the uniqueness theorem for the solution to (IP7). In fact this theorem is proved in [17] in a stonger form: the data $A_{1}\left(\alpha^{\prime}, \alpha\right)$ and $A_{2}\left(\alpha^{\prime}, \alpha\right)$ are asumed to be equal not for all $\alpha^{\prime}, \alpha \in S^{2}$ but only on a set $\widetilde{S}_{1}^{2} \times \widetilde{S}_{2}^{2}$, where $\widetilde{S}_{j}^{2}$ is an arbitrary small open subset of $S^{2}$.

In [24] the stability estimates for the solution to (IP7) with noisy data are obtained and an algorithm for finding such a solution is proposed.

The noisy data is an arbitrary function $A_{\varepsilon}\left(\alpha^{\prime}, \alpha\right)$, not necessarily a scattering amplitude, such that

$$
\sup _{\alpha^{\prime}, \alpha \in S^{2}}\left|A\left(\alpha^{\prime}, \alpha\right)-A_{\varepsilon}\left(\alpha^{\prime}, \alpha\right)\right|<\varepsilon .
$$

Given $A_{\varepsilon}\left(\alpha^{\prime}, \alpha\right)$, an algorithm for computing a quantity $\widehat{q}_{\varepsilon}$ is proposed in [24], such that

$$
\sup _{\xi \in \mathbb{R}^{3}}\left|\widehat{q}_{\varepsilon}-\widetilde{q}(\xi)\right|<c \frac{(\ln |\ln \varepsilon|)^{2}}{|\ln \varepsilon|} .
$$

where $c>0$ is a constant depending on the potential but not on $\varepsilon$,

$$
\widetilde{q}(\xi):=\int_{B_{a}} e^{i \xi \cdot x} q(x) d x .
$$

The constant $c$ in (7.14) can be chosen uniformly for all potentials $q \in Q_{a}$ which belong to a compact set in $L^{2}\left(B_{a}\right)$.

The right-hand side of (7.14) tends to zero as $\varepsilon \rightarrow 0$, but very slowly.

The author thinks that the rate (7.14) cannot be improved for the class $Q_{a}$, but this is not proved.

However, in [1] an example of two spherically symmetric piecewise-constant potentials $q(r)$ is constructed such that $\left|q_{1}-q_{2}\right|$ is of order 1 , maximal value of each of the potentials $q$ is of order 1 , the two potentials are quite different but they generate the set of the fixed-energy $(k=1)$ phase shifts $\left\{\delta_{\ell}^{(j)}\right\}_{\ell=0,1,2, \ldots}, j=1,2$, such that

$$
\delta_{\ell}^{(1)}=\delta_{\ell}^{(2)}, \quad 0 \leq \ell \leq 4, \quad\left|\delta_{\ell}^{(1)}-\delta_{\ell}^{(2)}\right| \leq 10^{-5}, \quad \forall \ell \geq 5 .
$$

In this example $\varepsilon \sim 10^{-5},(\ln |\ln \varepsilon|)^{2} \sim 2.59,|\ln \varepsilon| \sim 5$, so the right-hand side of (7.14) is of order 1 if one assumes $c$ to be of order 1 . 
Our point is: there are examples in which the left and the right sides of estimate (7.14) are of the same order of magnitude. Therefore estimate (7.14) is sharp.

7.2 Approximate inversion of fixed-energy phase shifts. Let us recall that $q(x)=q(r), r=|x|$ if and only if

$$
A\left(\alpha^{\prime}, \alpha, k\right)=A\left(\alpha^{\prime} \cdot \alpha, k\right) \text {. }
$$

It was well known for a long time that if $q=q(r)$ then $(7.17)$ holds. The converse was proved relatively recently in [32] (see also [17]).

If $q=q(r)$ then

$$
A\left(\alpha^{\prime}, \alpha\right)=\sum_{\ell=0}^{\infty} A_{\ell} Y_{\ell}\left(\alpha^{\prime}\right) \overline{Y_{\ell}(\alpha)},
$$

where $Y_{\ell}$ are orthonormal in $L^{2}\left(S^{2}\right)$ spherical harmonics, $Y_{\ell}=Y_{\ell m},-\ell \leq m \leq \ell$, summation with respect to $m$ is understood in (7.18) but not written for brevity, the numbers $A_{\ell}$ are related to the phase shifts $\delta_{\ell}$ by the formula

$$
A_{\ell}=4 \pi e^{i \delta_{\ell}} \sin \left(\delta_{\ell}\right) \quad(k=1)
$$

and the $S$-matrix is related to $A\left(\alpha^{\prime}, \alpha, k\right)$ by the formula

$$
S=I-\frac{k}{2 \pi i} A \text {. }
$$

If $q=q(r), r=|x|$, then the scattering solution $\psi(x, \alpha, k)$ can be written as

$$
\psi(x, \alpha, k)=\sum_{\ell=0}^{\infty} \frac{4 \pi}{k} i^{\ell} \frac{\psi_{\ell}(r, k)}{r} Y_{\ell}\left(x^{0}\right) \overline{Y_{\ell}(\alpha)}, \quad x^{0}:=\frac{x}{r} .
$$

The function $\psi_{\ell}(r, k)$ solves (uniquely) the equation

$$
\psi_{\ell}(r, k)=u_{\ell}(k r)-\int_{0}^{\infty} g_{\ell}(r, \rho) q(\rho) \psi_{\ell}(\rho, k) d \rho
$$

where

$$
u_{\ell}(k r):=\sqrt{\frac{\pi k r}{2}} J_{\ell+1 / 2}(k r), \quad v_{\ell}:=\sqrt{\frac{\pi k r}{2}} N_{\ell+1 / 2}(k r) .
$$

Here $J_{\ell}$ and $N_{\ell}$ are the Bessel and Neumann functions, and

$\psi_{0 \ell}$ and $f_{0 \ell}$ solve the equation

$$
\begin{gathered}
g_{\ell}(r, \rho):= \begin{cases}\frac{\varphi_{0 \ell}(k \rho) f_{0 \ell}(k r)}{F_{0 \ell}(k)}, & r \geq \rho, \\
\frac{\varphi_{0 \ell}(k r) f_{0 \ell}(k \rho)}{F_{0 \ell}(k)}, & r \leq \rho,\end{cases} \\
F_{0 \ell}(k)=\frac{e^{\frac{i \ell \pi}{2}}}{k^{\ell}},
\end{gathered}
$$

$$
\psi_{0 \ell}^{\prime \prime}+k^{2} \psi_{0 \ell}-\frac{\ell(\ell+1)}{r^{2}} \psi_{0 \ell}=0,
$$

and are defined by the conditions

$$
f_{0 \ell} \sim e^{i k r} \text { as } r \rightarrow+\infty
$$

SO

$$
f_{0 \ell}(k r)=i e^{\frac{i \ell \pi}{2}}\left(u_{\ell}+i v_{\ell}\right),
$$

where $u_{\ell}$ and $v_{\ell}$ are defined in (7.22), and

$$
\varphi_{0 \ell}(k r):=\frac{u_{\ell}(k r)}{k^{\ell+1}} .
$$


In [39] an approximate method was proposed recently for finding $q(r)$ given $\left\{\delta_{\ell}\right\}_{\ell=0,1,2, \ldots}$. In [40] numerical results based on this method are described.

In physics one often assumes $q(r)$ known for $r \geq a$ and then the data $\left\{\delta_{\ell}\right\}_{\ell=0,1,2, \ldots}$ allow one to calculate the data $\psi_{\ell}(a), \ell=0,1,2, \ldots$, by solving the equation

$$
\psi_{\ell}^{\prime \prime}+\psi_{\ell}-\frac{\ell(\ell+1)}{r^{2}} \psi_{\ell}-q(r) \psi_{\ell}=0, \quad r>a
$$

together with the condition

$$
\psi_{\ell} \sim e^{i \delta_{\ell}} \sin \left(r-\frac{\ell \pi}{2}+\delta_{\ell}\right), \quad r \rightarrow+\infty, \quad k=1,
$$

and assuming $q(r)$ known for $r>a$.

Problem (7.28) and (7.29) is a Cauchy problem with Cauchy data at infinity. Asymptotic formula (7.29) can be differentiated. If the data $\psi_{\ell}(a)$ are calculated and $\psi_{\ell}(r)$ for $r \geq a$ is found, then one uses the equation

$$
\psi_{\ell}(r)=\psi_{\ell}^{(0)}(r)-\int_{0}^{a} g_{\ell}(r, \rho) q(\rho) \psi_{\ell}(\rho) d \rho, \quad 0 \leq r \leq a,
$$

where $g_{\ell}$ is given in (7.23),

$$
\psi_{\ell}^{(0)}(r):=u_{\ell}(r)-\int_{a}^{\infty} g_{\ell}(r, \rho) q(\rho) \psi_{\ell}(\rho) d \rho,
$$

and $u_{\ell}(r)$ is given in $(7.22)$.

Put $r=a$ in (7.30) and get

$$
\int_{0}^{a} g_{\ell}(a, \rho) \psi_{\ell}(\rho) q(\rho) d \rho=\psi_{\ell}^{(0)}(a)-\psi_{\ell}(a):=b_{\ell}, \quad \ell=0,1,2, \ldots . .
$$

The numbers $b_{\ell}$ are known. If $q(\rho)$ is small or $\ell$ is large then the following approximation is justified:

$$
\psi_{\ell}(\rho) \approx \psi_{\ell}^{(0)}(\rho)
$$

Therefore, an approximation to equation (7.32) is:

$$
\int_{0}^{a} f_{\ell}(\rho) q(\rho) d \rho=b_{\ell}, \quad \ell=0,1,2, \ldots
$$

where

$$
f_{\ell}(\rho):=g_{\ell}(a, \rho) \psi_{\ell}^{(0)}(\rho) .
$$

The system of functions $\left\{f_{\ell}(\rho)\right\}$ is linearly independent.

Equations (7.34) constitute a moment problem which can be solved numerically for $q(\rho)([17, \mathrm{p} .209],[40])$.

8 A uniqueness theorem for inversion of fixed-energy phase shifts.

From Theorem 7.1 it follows that if $q=q(r) \in Q_{a}$ then the data

$$
\left\{\delta_{\ell}\right\} \quad \forall \ell=0,1,2, \ldots \quad k=1
$$

determine $q(r)$ uniquely [28].

Suppose a part of the phase shifts is known (this is the case in practice).

What part of the phase shifts is sufficient for the unique recovery of $q(r)$ ? 
In this section we answer this question following [23]. Define

$$
\mathcal{L}:=\left\{\ell: \sum_{\substack{\ell \neq 0 \\ \ell \in \mathcal{L}}} \frac{1}{\ell}=\infty\right\}
$$

to be any subset of nonnegative integers such that condition $(8.2)$ is satisfied.

For instance, $\mathcal{L}=\{2 \ell\}_{\ell=0,1,2, \ldots}$ or $\mathcal{L}=\{2 \ell+1\}_{\ell=0,1,2, \ldots}$ will be admissible.

Our main result is

Theorem 8.1 ([23]) If $q(r) \in Q_{a}$ and $L$ satisfies (8.2) then the set of fixedenergy phase shifts $\left\{\delta_{\ell}\right\}_{\forall \ell \in \mathcal{L}}$ determine $q(r)$ uniquely.

Let us outline basic steps of the proof.

Step 1. Derivation of the orthogonality relation:

If $q_{1}, q_{2} \in Q_{a}$ generate the same data then $p(r):=q_{1}-q_{2}$ satisfies the relation

$$
\int_{0}^{a} p(r) \psi_{\ell}^{(1)}(r) \psi_{\ell}^{(2)}(r) d r=0 \quad \forall \ell \in \mathcal{L} .
$$

Here $\psi_{\ell}^{(j)}(r)$ are defined in (7.20) and correspond to $q=q_{j}, j=1,2$. Note that Ramm's Theorem 7.1 yields the following conclusion: if

$$
\int_{0}^{a} p(r) \psi_{\ell}^{(1)}(r) \psi_{\ell}^{(2)}(r) d r=0 \quad \forall \ell \in \mathcal{L}
$$

then $p(r)=0$, and $q_{1}=q_{2}$.

Step 2. Since $\psi_{\ell}^{(j)}=c_{\ell}^{(j)} \varphi_{\ell}^{(j)}(r)$, where $c_{\ell}^{(j)}$ are some constants, relation (8.3) is equivalent to

$$
\int_{0}^{a} p(r) \varphi_{\ell}^{(1)}(r) \varphi_{\ell}^{(2)}(r) d r=0 \quad \forall \ell \in \mathcal{L}
$$

Here $\varphi_{\ell}^{(j)}(r)$ is the solution to $(7.28)$ with $q=q_{j}$, which satisfies the conditions:

$$
\varphi_{\ell}^{(j)}=\frac{r^{\ell+1}}{(2 \ell+1) ! !}+o\left(r^{\ell+1}\right), \quad r \rightarrow 0,
$$

and

$$
\varphi_{\ell}^{(j)}=\left|F_{\ell}^{(j)}\right| \sin \left(r-\frac{\ell \pi}{2}+\delta_{\ell}^{(j)}\right)+o(1), \quad r \rightarrow+\infty
$$

where $\left|F_{\ell}^{(j)}\right| \neq 0$ is a certain constant, and $\delta_{\ell}^{(j)}$ is the fixed-energy $(k=1)$ phase shift, which does not depend on $j$ by our assumption: $\delta_{\ell}^{(1)}=\delta_{\ell}^{(2)} \quad \forall \ell \in \mathcal{L}$.

We want to derive from (8.5) the relation (7.10). Since $q(x)=q(r), r=|x|$ in this section, relation (7.10) is equivalent to relation (8.12) (see below). The rest of this section contains this derivation of $(8.12)$.

We prove existence of the transformation kernel $K(r, \rho)$, independent of $\ell$, which sends functions $u_{\ell}(r)$, defined in $(7.22)$, into $\varphi_{\ell}(r)$ :

$$
\varphi_{\ell}(r)=u_{\ell}(r)+\int_{0}^{r} K(r, \rho) u_{\ell}(\rho) \frac{d \rho}{\rho^{2}}, \quad K(r, 0)=0
$$

Let

$$
\gamma_{\ell}:=\sqrt{\frac{2}{\pi}} \Gamma\left(\frac{1}{2}\right) 2^{\ell+\frac{1}{2}} \Gamma(\ell+1)
$$


where $\Gamma(z)$ is the gamma function,

$$
H(\ell):=\gamma_{\ell}^{2} \int_{0}^{a} p(r) u_{\ell}^{2}(r) d r,
$$

and

We prove that:

$$
h(\ell):=\gamma_{\ell}^{2} \int_{0}^{a} p(r) \varphi_{\ell}^{(1)}(r) \varphi_{\ell}^{(2)}(r) d r .
$$

$$
\{h(\ell)=0 \quad \forall \ell \in \mathcal{L}\} \Rightarrow\{h(\ell)=0 \quad \forall \ell=0,1,2, \ldots\} .
$$

If $h(\ell)=0, \quad \forall \ell=0,1,2, \ldots$, then (8.4) holds, and, by theorem 7.1, $p(r)=0$.

Thus, Theorem 8.1 follows.

The main claims to prove are:

1) Existence of the representation (8.8) and the estimate

$$
\int_{0}^{r}|K(r, \rho)| \frac{d \rho}{\rho}<c(r)<\infty \quad \forall r>0 .
$$

2) Implication(8.12)

Representation (8.8) was used in the physical literature ([3], [16]) but, to our knowledge, without any proof. Existence of transformation operators with kernels depending on $\ell$ was proved in the literature [43]. For our purposes it is important to have $K(r, \rho)$ independent of $\ell$.

Implication (8.12) will be established if one checks that $h(\ell)$ is a holomorphic function of $\ell$ in the half-plane $\prod_{+}:=\{\ell: \ell \in \mathbb{C}, \quad R e \ell>0\}$ which belongs to $\mathrm{N}-\mathrm{Class}$ (Nevanlinna class).

Definition 8.1 A function $h(\ell)$ holomorphic in $\prod_{+}$belongs to $N$-class iff

$$
\sup _{0<r<1} \int_{-\pi}^{\pi} \ln ^{+}\left|h\left(\frac{1-r e^{i \varphi}}{1+r e^{i \varphi}}\right)\right| d \varphi<\infty .
$$

Here

$$
u^{+}:=\left\{\begin{array}{l}
u \text { if } u \geq 0, \\
0 \text { if } u<0 .
\end{array}\right.
$$

The basic result we need in order to prove (8.12) is the following uniqueness theorem:

Proposition 8.1 If $h(\ell)$ belongs to $N$-class then (8.12) holds.

Proof This is an immediate consequence of the following:

Theorem ([41, p.334]): If $h(z)$ is holomorphic in $D_{1}:=\{z:|z|<1, \quad z \in \mathbb{C}\}$, $h(z)$ is of $N$-class in $D_{1}$, that is:

$$
\sup _{0<r<1} \int_{-\pi}^{\pi} \ln ^{+}\left|h\left(r e^{i \varphi}\right)\right| d \varphi<\infty,
$$

and

$$
h\left(z_{n}\right)=0, \quad h=1,2,3, \ldots,
$$

where

$$
\sum_{h=1}^{\infty}\left(1-\left|z_{n}\right|\right)=\infty
$$

then $h(z) \equiv 0$. 
The function $z=\frac{1-\ell}{1+\ell}$ maps conformally $\prod_{+}$onto $D_{1}, \ell=\frac{1-z}{1+z}$ and if $h(\ell)=$ $0 \forall \ell \in \mathcal{L}$, then $f(z):=h\left(\frac{1-z}{1+z}\right)$ is holomorphic in $D_{1}, f\left(z_{\ell}\right)=0$ for $\ell \in \mathcal{L}$ and $z_{\ell}:=\frac{1-\ell}{1+\ell}$, and

$$
\sum_{\ell \in \mathcal{L}}\left(1-\left|\frac{1-\ell}{1+\ell}\right|\right) \leq 1+\sum_{\ell \in \mathcal{L}}\left(1-\frac{\ell-1}{\ell+1}\right)=1+2 \sum_{\ell \in \mathcal{L}} \frac{1}{\ell+1}=\infty .
$$

From (8.18) and the above Theorem Proposition 8.1 follows.

Thus we need to check that function (8.11) belongs to N-class, that is, (8.14) holds.

So step 2, will be completed if one proves (8.8), (8.13) and (8.14).

Assuming (8.8) and (8.13), one proves (8.14) as follows:

i) First, one checks that (8.14) holds with $H(\ell)$ in place of $h(\ell)$.

ii) Secondly, using (8.8) one writes $h(\ell)$ as:

$$
\begin{aligned}
h(\ell) & =H(\ell)+\gamma_{\ell}^{2} \int_{0}^{r}\left[K_{1}(r, \rho)+K_{2}(r, \rho)\right] u_{\ell}(\rho) u_{\ell}(r) \frac{d \rho}{\rho^{2}}+ \\
& +\gamma_{\ell}^{2} \int_{0}^{r} \int_{0}^{r} \frac{d s}{s^{2}} \frac{d t}{t^{2}} K_{1}(r, t) K_{2}(r, s) u_{\ell}(t) u_{\ell}(s) .
\end{aligned}
$$

Let us now go through i) and ii) in detail.

In $[7,8.411 .8]$ one finds the formula:

$$
\gamma_{\ell} u_{\ell}(r)=r^{\ell+1} \int_{-1}^{1}\left(1-t^{2}\right)^{\ell} e^{i r t} d t
$$

where $\gamma_{\ell}$ is defined in (8.9).

From (8.20) and (8.10) one gets:

$$
|H(\ell)| \leq \int_{0}^{a} d r|p(r)| r^{2 \ell+2}\left|\int_{-1}^{1}\left(1-t^{2}\right)^{\ell} e^{i r t} d t\right|^{2} \leq c a^{2 \sigma}, \ell=\sigma+i \tau, \sigma \geq 0
$$

One can assume $a>1$ without loss of generality. Note that

$$
\ln ^{+}(a b) \leq \ln ^{+} a+\ln ^{+} b \quad \text { if } \quad a, b>0
$$

Thus (8.21) implies

$$
\begin{aligned}
& \int_{-\pi}^{\pi} \ln ^{+}\left|H\left(\frac{1-r e^{i \varphi}}{1+r e^{i l}}\right)\right| d \varphi \leq \int_{-\pi}^{\pi} \ln ^{+}\left|c a^{2 \operatorname{Re} \frac{1-r e^{i \varphi}}{1+r i \varphi}}\right| d \varphi \\
& \quad \leq|\ln c|+2 \ln a \int_{-\pi}^{\pi}\left|\operatorname{Re} \frac{1-r e^{i \varphi}}{1+r e^{i \varphi}}\right| d \varphi \\
& \quad \leq|\ln c|+2 \ln a \int_{-\pi}^{\pi} \frac{1-r^{2}}{1+2 r \cos \varphi+r^{2}} d \varphi \leq|\ln c|+4 \pi \ln a<\infty
\end{aligned}
$$

Here we have used the known formula:

$$
\int_{-\pi}^{\pi} \frac{d \varphi}{1+2 r \cos \varphi+r^{2}}=\frac{2 \pi}{1-r^{2}}, \quad 0<r<1 .
$$

Thus, we have checked that $H(\ell) \in N\left(\prod_{+}\right)$, that is, (8.14) holds for $H(\ell)$. 
Consider the first integral, call it $I_{1}(\ell)$, in (8.19). One has, using (8.20) and $(8.13)$,

$$
\begin{gathered}
\left|I_{1}(\ell)\right| \leq \int_{0}^{r} d \rho \rho^{-1}\left(\left|K_{1}(r, \rho)\right|+\left|K_{2}(r, \rho)\right|\right) r^{\ell+1} \rho^{\ell} \leq c(a) a^{2 \sigma}, \\
\quad \ell=\sigma+i \tau, \quad \sigma \geq 0 .
\end{gathered}
$$
(8.23).

Therefore one checks that $I_{1}(\ell)$ satisfies (8.14) (with $I_{1}$ in place of $h$ ) as in

The second integral in $(8.19)$, call it $I_{2}(\ell)$, is estimated similarly: one uses (8.20) and (8.13) and obtains the following estimate:

$$
\left|I_{2}(\ell)\right| \leq c(a) a^{2 \sigma}, \quad \ell=\sigma+i \tau, \quad \sigma \geq 0 .
$$

Thus, we have proved that $h(\ell) \in N\left(\prod_{+}\right)$.

To complete the proof one has to derive (8.4) and check (8.13).

Derivation of (8.4): Subtract from (7.28) with $q=q_{1}$ this equation with $q=q_{2}$ and get:

$$
w^{\prime \prime}+w-\frac{\ell(\ell+1)}{r^{2}} w-q_{1} w=p \psi_{\ell}^{(2)},
$$

where

$$
p:=q_{1}-q_{2}, \quad w:=\psi_{\ell}^{(1)}(r)-\psi_{\ell}^{(2)}(r) .
$$

Multiply $(8.27)$ by $\psi_{\ell}^{(1)}(r)$, integrate over $[0, \infty)$, and then by parts on the left, and get

$$
\left.\left(w^{\prime} \psi_{\ell}^{(1)}-w \psi_{\ell}^{(1)^{\prime}}\right)\right|_{0} ^{\infty}=\quad \int_{0}^{a} p \psi_{\ell}^{(2)} \psi_{\ell}^{(1)} d r, \quad \ell \in \mathcal{L}
$$

By the assumption $\delta_{\ell}^{(1)}=\delta_{\ell}^{(2)}$ if $\ell \in \mathcal{L}$, so $w$ and $w^{\prime}$ vanish at infinity. At $r=0$ the left-hand side of (8.29) vanishes since

$$
\psi_{\ell}^{(j)}(r)=O\left(r^{\ell+1}\right) \quad \text { as } r \rightarrow 0 .
$$

Thus (8.29) implies (8.4).

Derivation of the representation (8.8) and of the estimate (8.13). One can prove [23] that the kernel $K(r, \rho)$ of the transformation operator must solve the Goursat-type problem

$$
\begin{gathered}
r^{2} K_{r r}(r, \rho)-\rho^{2} K_{\rho \rho}(r, \rho)+\left[r^{2}-r^{2} q(r)-\rho^{2}\right] K(r, \rho)=0, \quad 0 \leq \rho \leq r, \\
K(r, r)=\frac{r}{2} \int_{0}^{r} s q(s) d s:=g(r), \\
K(r, 0)=0,
\end{gathered}
$$

and conversely: the solution to this Goursat-type problem is the kernel of the transformation operator (8.8).

The difficulty in a study of the problem comes from the fact that the coefficients in front of the second derivatives degenerate at $\rho=0, r=0$.

To overcome this difficulty let us introduce new variables:

$$
\xi=\ln r+\ln \rho, \quad \eta=\ln r-\ln \rho .
$$

Put

$$
K(r, \rho):=B(\xi, \eta)
$$


Then (8.31)-(8.33) becomes

$$
\begin{gathered}
B_{\xi \eta}-\frac{1}{2} B_{\eta}+Q(\xi, \eta) B=0, \quad \eta \geq 0, \quad-\infty<\xi<\infty, \\
B(\xi, 0)=g\left(e^{\frac{\xi}{2}}\right):=G(\xi), \\
B(-\infty, \eta)=0, \quad \eta \geq 0,
\end{gathered}
$$

where $g(r)$ is defined in (8.32) and

$$
Q(\xi, \eta):=\frac{1}{4}\left[e^{\xi+\eta}-e^{\xi+\eta} q\left(e^{\frac{\xi+\eta}{2}}\right)-e^{\xi-\eta}\right]
$$

Note that

$$
\begin{gathered}
\sup _{-\infty<\xi<\infty} e^{-\frac{\xi}{2}} G(\xi)<c, \\
\sup _{0 \leq \eta \leq B} \int_{-\infty}^{A}|Q(s, \eta)| d s \leq c(A, B),
\end{gathered}
$$

for any $A \in \mathbb{R}$ and any $B>0$, where $c(A, B)>0$ is some constant.

Let

Write (8.36)-(8.38) as

$$
L(\xi, \eta):=B(\xi, \eta) e^{-\frac{\xi}{2}}
$$

$$
\begin{gathered}
L_{\xi \eta}+Q(\xi, \eta) L=0, \quad \eta \geq 0, \quad-\infty<\xi<\infty \\
L(\xi, 0)=e^{-\frac{\xi}{2}} G(\xi):=b(\xi) ; \quad L(-\infty, \eta)=0, \quad \eta \geq 0 .
\end{gathered}
$$

Integrate (8.43) with respect to $\eta$ and use (8.44), and then integrate with respect to $\xi$ to get:

$$
L=V L+b, \quad V L:=-\int_{-\infty}^{\xi} d s \int_{0}^{\eta} d t Q(s, t) L(s, t) .
$$

Consider (8.45) in the Banach space $X$ of continous function $L(\xi, \eta)$ defined for $\eta \geq 0,-\infty<\xi<\infty$, with the norm

$$
\|L\|:=\|L\|_{A B}:=\sup _{\substack{0 \leq t \leq B \\-\infty<s \leq A}}\left(e^{-\gamma t}|L(s, t)|\right)<\infty,
$$

where $\gamma=\gamma(A, B)>0$ is chosen so that the operator $V$ is a contraction mapping in $X$. Let us estimate $\|V\|$ :

$$
\begin{aligned}
\|V L\| & \leq \sup _{\substack{-\infty<\xi \leq A \\
0 \leq \eta \leq B}} \int_{-\infty}^{\xi} d s \int_{0}^{\eta} d t|Q(s, t)| e^{-\gamma(\eta-t)} e^{-\gamma t}|L(s, t)| \\
& \leq\|L\| \sup _{\substack{-\infty<\xi \leq A \\
0 \leq \eta \leq B}} \int_{-\infty}^{\xi} d s \int_{0}^{\eta} d t\left(2 e^{s+t}+e^{s+t}\left|q\left(e^{\frac{s+t}{2}}\right)\right|\right) e^{-\gamma(\eta-t)} \leq \frac{c}{\gamma}\|L\|,
\end{aligned}
$$

where $c>0$ is a constant which depends on $A, B$, and on $\int_{0}^{a} r|q(r)| d r$.

If $\gamma>c$ then $V$ is a contraction mapping in $X$ and equation (8.45) has a unique solution in $X$ for any $-\infty<A<\infty$ and $B>0$.

Let us now prove that estimate (8.13) holds for the constructed function $K(r, \rho)$.

One has

$$
\int_{0}^{r}|K(r, \rho)| \rho^{-1} d \rho=r \int_{0}^{\infty}|L(2 \ln r-\eta, \eta)| e^{-\frac{\eta}{2}} d \eta<\infty
$$


The last inequality follows from the estimate:

$$
|L(\xi, \eta)| \leq c e^{\left(2+\varepsilon_{1}\right)\left[\eta \mu_{1}(\xi+\eta)\right]^{\frac{1}{2}+\varepsilon_{2}}}
$$

where $\varepsilon_{1}$ and $\varepsilon_{2}>0$ are arbitrarily small numbers,

$$
\mu_{1}(\xi):=\int_{-\infty}^{\xi} d s \mu(s), \quad \mu(s):=\frac{e^{s}}{2}\left(1+\left|q\left(e^{\frac{s}{2}}\right)\right|\right)
$$

The proof of Theorem 8.1 is complete when (8.49) is proved.

Lemma 8.1 Estimate (8.49) holds.

Proof From (8.45) one gets

$$
m(\xi, \eta) \leq c+W m
$$

where

$$
c_{0}:=\sup _{-\infty<\xi<\infty}|b(\xi)| \leq \frac{1}{2} \int_{0}^{a} s(q(s) d s, \quad m(\xi, \eta):=|L(\xi, \eta)|,
$$

and

$$
W m:=\int_{-\infty}^{\xi} d s \int_{0}^{\eta} d t \mu(s+t) m(s, t)
$$

Without loss of generality we can take $c_{0}=1$ in (8.51): If (8.49) is derived from (8.51) with $c_{0}=1$, it will hold for any $c_{0}>0$ (with a different $c$ in (8.49)). Thus, consider (8.51) with $c_{0}=1$ and solve this inequality by iterations.

One has

$$
W 1=\int_{-\infty}^{\xi} d s \int_{0}^{\eta} \mu(s+t) d t=\int_{0}^{\eta} \mu_{1}(\xi+t) d t \leq \eta \mu_{1}(\xi+\eta) .
$$

One can prove by induction that

$$
W^{n} 1 \leq \frac{\eta^{n}}{h !} \frac{\mu_{1}^{n}(\xi+\eta)}{n !} .
$$

Therefore (8.57) with $c_{0}=1$ implies

$$
m(\xi, \eta) \leq 1+\sum_{h=1}^{\infty} \frac{\eta^{n}}{n !} \frac{\mu_{1}^{n}(\xi+\eta)}{n !} .
$$

Consider

$$
F(z):=1+\sum_{n=1}^{\infty} \frac{z^{n}}{(n !)^{2}} .
$$

This is an entire function of order $\frac{1}{2}$ and type 2 .

Thus

$$
|F(z)| \leq c e^{\left(2+\varepsilon_{1}\right)|z|^{\frac{1}{2}+\varepsilon_{2}}} .
$$

From (8.56) and (8.58) estimate (8.49) follows.

Lemma 8.1 is proved.

Theorem 8.1 is proved. 


\section{Discussion of the Newton-Sabatier procedure for recovery of $q(r)$} from the fixed-energy phase shifts

In [3] and [16] the following procedure is proposed for inversion of the fixedenergy phase shifts for $q(r)$. We take $k=1$ in what follows.

Step 1. Given $\left\{\delta_{\ell}\right\}_{\forall \ell=0,1,2, \ldots}$ one solves an infinite linear algebraic system $((12.2 .7)$ in $[3])$

$$
\tan \delta_{\ell}=\sum_{\ell^{\prime}=0}^{\infty} M_{\ell \ell^{\prime}}\left(1+\tan \delta_{\ell} \tan \delta_{\ell^{\prime}}\right) a_{\ell^{\prime}}
$$

for constants $a_{\ell}$. Here

$$
M_{\ell \ell^{\prime}}=\left\{\begin{array}{l}
0 \text { if }\left|\ell-\ell^{\prime}\right| \text { is even or zero, } \\
\frac{1}{\left(\ell^{\prime}+\frac{1}{2}\right)^{2}-\left(\ell+\frac{1}{2}\right)^{2}} \quad \text { if }\left|\ell-\ell^{\prime}\right| \text { is odd. }
\end{array}\right.
$$

Assuming that (9.1) is solvable and $a_{\ell}$ are found, one calculates (see formula (12.2.8) in $[3])$

$$
c_{\ell}=a_{\ell}\left(1+\tan ^{2} \delta_{\ell}\right)\left\{1-\frac{\pi a_{\ell}\left(1+\tan ^{2} \delta_{\ell}\right)}{4 \ell+2}-\sum_{\ell^{\prime}=0}^{\infty} M_{\ell \ell^{\prime}} a_{\ell^{\prime}}\left(\tan \delta_{\ell^{\prime}}-\tan \delta_{\ell}\right)\right\}^{-1}
$$

Step 2. If $c_{\ell}$ are found, one solves the equation for $K(r, \rho)$ (see formula (12.1.12) in $[3])$

$$
K(r, s)=f(r, s)-\int_{0}^{r} K(r, t) f(t, s) t^{-2} d t
$$

where

$$
f(r, s):=\sum_{\ell=0}^{\infty} c_{\ell} u_{\ell}(r) u_{\ell}(s)
$$

and $u_{\ell}(r)$ are defined in (7.22).

Note that in this section the notations from [3] are used and by this reason the kernel $K(r, t)$ in formulas (9.4) and (9.6) differs by sign from the kernel $K(r, \rho)$ in formula (8.8). This explains the minus sign in formula (9.6).

Assuming that (9.4) is solvable for all $r>0$, one calculates

$$
q(r)=-\frac{2}{r} \frac{d}{d r} \quad \frac{K(r, r)}{r} .
$$

Note that if (9.4) is not solvable for some $r=r>0$, then the procedure breaks down because the potential (9.6) is no longer locally integrable in $(0, \infty)$. In [16] it is argued that for sufficiently small $a$ equation (9.4) is uniquely solvable by iterations for all $0<r<a$, but no discussion of the global solvability, that is, solvability for all $r>0$, is given. It is assumed in [3] and [16] that the sequence $\left\{c_{\ell}\right\}$ in (9.3) does not grow fast. In $([3,(12.2 .2)])$ the following is assumed:

$$
\sum_{\ell=1}^{\infty}\left|c_{\ell}\right| \ell^{-2}<\infty
$$

Under this assumption, and also under much weaker assumption

$$
\left|c_{\ell}\right| \leq c e^{b \ell}
$$


for some $b>0$ arbitrary large fixed, one can prove that the kernel (9.5) is an entire function of $r$ and $s$. This follows from the known asymptotics of $u_{\ell}(r)$ as $\ell \rightarrow \infty$ :

$$
u_{\ell}(r)=\sqrt{\frac{r}{2}}\left(\frac{e r}{2 \ell+1}\right)^{\frac{2 \ell+1}{2}} \frac{1}{\sqrt{2 \ell+1}}[1+o(1)], \quad \ell \rightarrow+\infty .
$$

Thus, equation (9.4) is a Fredholm-type equation with kernel which is an entire function of $r$ and $s$. Since $K(r, 0)=0$ and $f(r, s)=f(s, r)=0$ at $s=0$, equation (9.4) is a Fredholm equation in the space of continuous functions $C(0, r)$ for any $r>0$.

If (9.4) is uniquely solvable for all $r>0$, then one can prove the following:

Claim $K(r, s)$ is an analytic function of $r$ and $s$ in a neighborhood $\Delta$ of the positive semiaxis $(0, \infty)$ on the complex plane of the variables $r$ and $s$.

This claim is proved below, at the end of this section.

Therefore the potential (9.6) has to have the following:

Property $P: q(r)$ is an analytic function in $\Delta$ with a possible simple pole at $r=0$.

Most of the potentials do not have this property. Therefore, if one takes any potential which does not have property $P$, for example, a compactly supported potential $q(r)$, and if it will be possible to carry through the Newton-Sabatier procedure, that is, (9.1) will be solvable for $a_{\ell}$ and generate $c_{\ell}$ by formula (9.3) such that (9.7) or $\left(9.7^{\prime}\right)$ hold, and (9.4) will be uniquely solvable for all $r>0$ then the potential (9.6), which this procedure yields, cannot coincide with the potential with which we started.

An important open question is: assuming that the Newton-Sabatier procedure can be carried through, is it true that the reconstructed potential (9.6) generates the scattering data, that is, the set of the fixed-energy phase shifts $\left\{\delta_{\ell}\right\}$ with which we started?

In [3, pp.203-205] it is claimed that this is the case. But the arguments in [3] are not convincing. In particular, the author was not able to verify equation (12.3.12) in [3] and in the argument on p.205 it is not clear why $A_{\ell}^{\prime}$ and $\delta_{\ell}^{\prime}$ satisfy the same equation (12.2.5) as the orginal $A_{\ell}$ and $\delta_{\ell}$.

In fact, it is claimed in $[3,(12.5 .6)]$ that $\delta_{\ell}^{\prime}=O\left(\frac{1}{\ell}\right)$, while it is known [37] that if $q(r)=0$ for $r>a$ and $q(r)$ does not change sign in some interval $(a-\varepsilon, a), q \neq 0$ if $r \in(a-\varepsilon, a)$, where $\varepsilon>0$ is arbitrarily small fixed number, then

$$
\lim _{\ell \rightarrow \infty}\left(\frac{2 \ell}{e}\left|\delta_{\ell}\right|^{\frac{1}{2 \ell}}\right)=a \text {. }
$$

It follows from (9.9) that for the above potentials the phase shifts decay very fast as $\ell \rightarrow \infty$, much faster that $\frac{1}{\ell}$. Therefore $\delta_{\ell}^{\prime}$ decaying at the rate $\frac{1}{\ell}$ cannot be equal to $\delta_{\ell}$, as it is claimed in [3, p.205], because formula (9.9) implies:

$$
\left|\delta_{\ell}\right| \sim\left(\frac{e a[1+o(1)]}{2 \ell}\right)^{2 \ell} .
$$

It is claimed in [3], p.105, that the Newton-Sabatier procedure leads to "one (only one) potential which decreases faster that $r^{-\frac{3}{2}}$ " and yields the original phase shifts. However, if one starts with a compactly supported integrable potential (or any other rapidly decaying potential which does not have property $P$ and belongs to the class of the potentials for which the uniqueness of the solution to inverse 
scattering problem with fixed-energy data is established), then the Newton-Sabatier procedure will not lead to this potential as is proved in this section. Therefore, either the potential which the Newton-Sabatier procedure yields does not produce the original phase shifts, or there are at least two potentials which produce the same phase shifts.

A more detailed analysis of the Newton-Sabatier procedure is given by the author in [1].

Proof of the claim. The idea of the proof is to consider $r$ in (9.4) as a parameter and to reduce (9.4) to a Fredholm-type equation with constant integration limits and kernel depending on the parameter $r$. Let $t=r \tau, s=r \sigma$,

$$
\frac{K(r, r \tau)}{\tau}:=b(\tau ; r), \quad \frac{f(r \tau, r \sigma)}{r \tau \sigma}:=a(\sigma, \tau, r), \quad \frac{f(r, \sigma)}{\sigma}:=g(\sigma ; r)
$$

Then (9.4) can be written as:

$$
b(\sigma ; r)=g(r ; \sigma)-\int_{0}^{1} a(\sigma, \tau ; r) b(\tau ; r) d \tau
$$

Equation (9.11) is equivalent to (9.4), it is a Fredholm-type equation with kernel $a(\sigma, \tau ; r)$ which is an entire function of $\sigma$ and of the parameter $r$. The free term $g(r, \sigma)$ is an entire function of $r$ and $\sigma$. This equation is uniquely solvable for all $r>0$ by the assumption. Therefore its solution $b(\sigma ; r)$ is an analytic function of $r$ in a neighborhood of any point $r>0$, and it is an entire function of $\sigma$ [35]. Thus $K(r, r)=b(1, r)$ is an analytic function of $r$ in a neighborhood of the positive semiaxis $(0, \infty)$.

\section{Reduction of some inverse problems to an overdetermined Cauchy problem}

Consider, for example, the classical problem of finding $q(x)$ from the knowledge of two spectra. Let $u$ solve (1.1) on the interval $[0,1]$ and satisfy the boundary conditions

$$
u(0)=u(1)=0
$$

and let the corresponding eigenvalues $k_{n}^{2}:=\lambda_{n}, n=1,2, \ldots$, be given. If

$$
u(0)=u^{\prime}(1)+h u(1)=0,
$$

then the corresponding eigenvalues are $\mu_{n}, \quad n=1,2, \ldots$

The inverse problem (IP10) is:

Given the two spectra $\left\{\lambda_{n}\right\} \cup\left\{\mu_{n}\right\}, n=1,2,3, \ldots$, find $q(x)$ (and $h$ in (10.3)).

Let us reduce this problem to an overdetermined Cauchy problem. Let

$$
u(x, k)=\frac{\sin (k x)}{k}+\int_{0}^{x} K(x, y) \frac{\sin (k y)}{k} d y:=(I+K)\left(\frac{\sin (k x)}{k}\right)
$$

solve (1.1). Then (10.1) and (10.2) imply:

$$
0=\frac{\sin \sqrt{\lambda_{n}}}{\sqrt{\lambda_{n}}}+\int_{0}^{1} K(1, y) \frac{\sin \left(\sqrt{\lambda_{n}} y\right)}{\sqrt{\lambda_{n}}} d y, \quad n=1,2, \ldots
$$

and

$$
0=\cos \sqrt{\mu_{n}}+K(1,1) \frac{\sin \sqrt{\mu_{n}}}{\sqrt{\mu_{n}}}+\int_{0}^{1} K_{x}(1, y) \frac{\sin \left(\sqrt{\mu_{n}} y\right)}{\sqrt{\mu_{n}}} d y, \quad n=1,2, \ldots
$$


It is known [14] that

$$
\lambda_{n}=(n \pi)^{2}+c_{0}+o(1), \quad n \rightarrow \infty,
$$

and

$$
\mu_{n}=\pi^{2}\left(n+\frac{1}{2}\right)^{2}+c_{1}+o(1), \quad n \rightarrow \infty,
$$

where $c_{0}$ and $c_{1}$ can be calculated explicitly, they are proportional to $\int_{0}^{1} q(x) d x$.

Therefore,

$$
\sqrt{\lambda_{n}}=\pi n\left[1+O\left(\frac{1}{n^{2}}\right)\right], \quad \sqrt{\mu_{n}}=\pi\left(n+\frac{1}{2}\right)\left[1+O\left(\frac{1}{n^{2}}\right)\right], \quad n \rightarrow \infty .
$$

It is known [10] that if (10.9) holds then each of the systems of functions:

$$
\left\{\sin \left(\sqrt{\lambda_{n}} x\right)\right\}_{n=1,2, \ldots}, \quad\left\{\sin \left(\sqrt{\mu_{n}} x\right)\right\}_{n=1,2, \ldots}
$$

is complete in $L^{2}[0,1]$.

Therefore equation (10.5) determines uniquely $\{K(1, y)\}_{0 \leq y \leq 1}$ and can be used for an efficient numerical procedure for finding $K(1, y)$ given the set $\left\{\lambda_{n}\right\}_{n=1,2, \ldots}$ Note that the system $\left\{\sin \left(\sqrt{\lambda_{n}} x\right)\right\}_{n=1,2, \ldots}$ forms a Riesz basis of $L^{2}[0,1]$ since the operator $I+K$, defined by (10.4) is boundedly invertible and the system $\left\{u\left(x, \sqrt{\lambda_{n}}\right)\right\}_{n=1,2, \ldots}$ forms an orthornormal basis of $L^{2}(0,1)$.

Equation (10.6) determines uniquely $\left\{K_{x}(1, y)\right\}_{0 \leq y \leq 1}$ if $\left\{\mu_{n}\right\}_{n=1,2, \ldots}$ are known. Indeed, the argument is the same as above. The constant $K(1,1)$ is uniquely determined by the data $\left\{\mu_{n}\right\}_{n=1,2, \ldots}$ because, by formula (5.41),

$$
K(1,1)=\frac{1}{2} \int_{0}^{1} q(x) d x .
$$

We have arrived at the following overdetermined Cauchy problem:

Given the Cauchy data

$$
\left\{K(1, y), K_{x}(1, y)\right\}_{0 \leq y \leq 1}
$$

and the equations (5.40) - (5.41), find $q(x)$.

It is easy to derive [22, eq. (4.36)] for the unknown vector function

$$
U:=\left(\begin{array}{c}
q(x) \\
K(x, y)
\end{array}\right)
$$

the following equation

$$
U=W(U)+h,
$$

where

$$
W(U):=\left(\begin{array}{c}
-2 \int_{x}^{1} q(s) K(s, 2 x-s) d s \\
\frac{1}{2} \int_{D_{x y}} q(s) K(s, t) d s d t
\end{array}\right),
$$

$D_{x y}$ is the region bounded by the straight lines on the $(s, t)$ plane: $s=1, \quad t-y=$ $s-x$ and $t-y=-(s-x)$, and

$$
\begin{gathered}
h:=\left(\begin{array}{l}
f \\
g
\end{array}\right), \\
f(x):=2\left[K_{y}(1,2 x-1)+K_{x}(1,2 x-1)\right], \\
g(x, y):=\frac{K(1, y+x-1)+K(1, y-x+1)}{2}-\frac{1}{2} \int_{y+x-1}^{y-x+1} K_{s}(1, t) d t .
\end{gathered}
$$


Note that $f$ and $g$ are computable from data (10.12), and (10.14) is a nonlinear equation for $q(x)$ and $K(x, y)$.

Consider the iterative process:

$$
U_{n+1}=W\left(U_{n}\right)+h, \quad U_{0}=h .
$$

Assume that

$$
q(x)=0 \text { for } x>1, \quad q=\bar{q}, \quad q \in L^{\infty}[0,1] .
$$

Let $x_{0} \in(0,1)$ and define the space of functions:

$$
L\left(x_{0}\right):=L^{\infty}\left(x_{0}, 1\right) \times L^{\infty}\left(\Delta_{x_{0}}\right),
$$

where

$$
\Delta_{x_{0}}:=\left\{x, y: x_{0} \leq x \leq 1, \quad 0 \leq|y| \leq x\right\}
$$

Denote

$$
\|u\|:=\operatorname{esssup}_{x_{0} \leq x \leq 1}|q(x)|+\sup _{x, y \in \Delta_{x o}}|K(x, y)| .
$$

Let

$$
\|h\| \leq R .
$$

Theorem 10.1 ([22]) Let (10.17), (10.18), (10.20), and (10.24) hold, and choose any $\widetilde{R}>R$. Then process (10.19) converges in $L\left(x_{0}\right)$ at the rate of a geometrical progression for any $x_{0} \in(1-\mu, 1)$, where

$$
\mu:=\min \left(\frac{8(\widetilde{R}-R)}{5 \widetilde{R}^{2}}, \frac{2}{5 \widetilde{R}}\right) .
$$

One has

$$
\lim _{n \rightarrow \infty} U_{n}=\left(\begin{array}{c}
q(x) \\
K(x, y)
\end{array}\right), x, y \in \Delta_{x_{0}} .
$$

If one starts with the data

$$
\left\{K\left(x_{0}, y\right), \quad K_{x}\left(x_{0}, y\right)\right\}_{0 \leq|y| \leq x_{0}},
$$

replaces in (10.19) $h$ by $h_{0}:=\left(\begin{array}{l}f_{0} \\ g_{0}\end{array}\right)$, where $f_{0}$ and $g_{0}$ are calculated by formulas (1.17) and (1.18) in which the first argument in $K(1, y), x=1$, is replaced by $x=x_{0}$, then the iterative process (10.19) with the new $h=h_{0}$, in the new space

$$
L\left(x_{1}\right):=L^{\infty}\left(x_{1}, x_{0}\right) \times L^{\infty}\left(\Delta_{x_{1}}\right),
$$

with

converges to $\left(\begin{array}{c}q(x) \\ K(x, y)\end{array}\right)$ in $L\left(x_{1}\right)$.

$$
\Delta_{x_{1}}=\left\{x, y: x_{1} \leq x \leq x_{0}, \quad 0 \leq|y| \leq x\right\},
$$

In finite number of steps one can uniquely reconstruct $q(x)$ on [0,1] from the data (10.12) using (10.19).

Proof First, we prove convergence of the process (10.19) in $L\left(x_{0}\right)$.

The proof makes it clear that this process will converge in $L\left(x_{1}\right)$ and that in final number of steps one recovers $q(x)$ uniquely on $[0,1]$. Let $B(R):=\{U:\|U\| \leq \widetilde{R}$, $\left.U \in L\left(x_{0}\right)\right\}, \widetilde{R}>R$.

Let us start with

Lemma 10.1 The map $U \in W(U)+h$ maps $B(\widetilde{R})$ into itself and is a contraction on $B(\widetilde{R})$ if $x_{0} \in(1-\mu, 1), \mu:=\min \left(\frac{8(\widetilde{R}-R)}{5 \widetilde{R}^{2}}, \frac{2}{5 \widetilde{R}}\right)$. 
Proof of the lemma. Let $U=\left(\begin{array}{c}q_{1} \\ K_{1}\end{array}\right), V=\left(\begin{array}{c}q_{2} \\ K_{2}\end{array}\right)$ One has:

$$
\begin{aligned}
\|W(U)-W(V)\| & \leq\left\|\begin{array}{l}
2 \int_{x}^{1}\left(\left|q_{1}-q_{2}\right|\left|K_{1}\right|+\left|q_{2}\right|\left|K_{1}-K_{2}\right|\right) d s \\
\frac{1}{2} \int_{D_{x y}}\left(\left|q_{1}-q_{2}\right|\left|K_{1}\right|+\left|q_{2}\right|\left|K_{1}-K_{2}\right|\right) d s d t
\end{array}\right\| \\
& \leq\|U-V\|\left[2\left(1-x_{0}\right) \widetilde{R}+\frac{\widetilde{R}}{2}\left(1-x_{0}\right)^{2}\right] \leq\|U-V\|\left(1-x_{0}\right) \frac{5}{2} \widetilde{R} .
\end{aligned}
$$

Here we have used the estimate $\left(1-x_{0}\right)^{2}<1-x_{0}$ and the assumption $\|U\| \leq \widetilde{R}$, $\|V\| \leq \widetilde{R}$.

If

$$
1-x_{0}<\frac{2}{5 \widetilde{R}}
$$

then $W$ is a contraction on $B(\widetilde{R})$.

Let us check that the map $T(U)=W(U)+h$ maps $B(\widetilde{R})$ into itself if $1-x_{0}<\mu$.

Using the inequality $a b \leq \frac{\widetilde{R}^{2}}{4}$ if $a+b=\widetilde{R}, a, b \geq 0$, one gets:

$$
\begin{aligned}
& \|W(U)+h\| \leq\|W(U)\|+\|h\| \\
& \quad \leq 2\left(1-x_{0}\right) \frac{\widetilde{R}^{2}}{4}+\frac{1}{2}\left(1-x_{0}\right)^{2} \frac{\widetilde{R}^{2}}{4}+R \\
& \leq \frac{5}{2}\left(1-x_{0}\right) \frac{\widetilde{R}^{2}}{4}+R<\widetilde{R} \text { if } 1-x_{0}<\frac{8(\widetilde{R}-R)}{5 \widetilde{R}^{2}} .
\end{aligned}
$$

Thus if

$$
\mu=\min \left(\frac{8(\widetilde{R}-R)}{5 \widetilde{R}^{2}}, \quad \frac{2}{5 \widetilde{R}}\right)
$$

then the map $U \rightarrow W(U)+h$ is a contraction on $B(\widetilde{R})$ in the space $L\left(x_{0}\right)$. Lemma 10.1 is proved.

From Lemma 10.1 it follows that process (10.19) converges at the rate of geometrical progression with common ratio (10.30). The solution to (10.14) is therefore unique in $L\left(x_{0}\right)$.

Since for the data $h$ which comes from a potential $q \in L^{\infty}(0,1)$ the vector $\left(\begin{array}{c}q(x) \\ K(x, y)\end{array}\right)$ solves $(10.14)$ in $L\left(x_{0}\right)$, it follows that this vector satisfies (10.25). Thus, process (10.19) allows one to reconstruct $q(x)$ on the interval from data (10.12), $x_{0}=1-\mu$, where $\mu$ is defined in (10.30).

If $q(x)$ and $K(x, y)$ are found on the interval $\left(x_{0}, 1\right)$, then $K\left(x_{0}, y\right)$ and $K_{x}\left(x_{0}, y\right)$ can be calculated for $0 \leq|y| \leq x_{0}$. Now one can repeat the argument for the interval $\left(x_{1}, x_{0}\right), \quad x_{0}-x_{1}<\mu$, and in finite number of the steps recover $q(x)$ on the whole interval $[0,1]$.

Note that one can use a fixed $\mu$ if one chooses $R$ so that (10.24) holds for $h$ defined by (10.16) and (10.17) with any $x \in L^{\infty}[0,1]$. Such $R$ does exist if $q \in L^{\infty}[0,1]$.

Theorem 10.1 is proved.

Remark 10.1 Other inverse problems have been reduced to the overdetermined Cauchy problem studied in this section (see [42], [22], [17]). The idea of this reduction was used in [42] for a numerical solution of some inverse problems. 


\section{Representation of I-function}

The $I(k)$ function (2.1) equals to the Weyl function (2.3). Our aim in this section is to derive the following formula ([19]):

$$
I(k)=i k+\sum_{j=0}^{J} \frac{i r_{j}}{k-i k_{j}}+\widetilde{a}, \quad \widetilde{a}:=\int_{0}^{\infty} a(t) e^{i k t} d t,
$$

where $k_{0}:=0, \quad r_{j}=$ const $>0,1 \leq j \leq J ; r_{0}>0$ if and only if $f(0)=0$; $a(t)=\overline{a(t)}$ is a real-valued function,

$$
\begin{gathered}
a(t) \in L^{1}\left(\mathbb{R}_{+}\right) \text {if } f(0) \neq 0 \text { and } q(x) \in L_{1,1}\left(\mathbb{R}_{+}\right), \\
a(t) \in L^{1}\left(\mathbb{R}_{+}\right) \text {if } f(0)=0 \text { and } q \in L_{1,3}\left(\mathbb{R}_{+}\right) .
\end{gathered}
$$

We will discuss the inverse problem of finding $q(x)$ given $I(k) \forall k>0$. Uniqueness of the solution to this problem is proved in Theorem 2.1. Here we discuss a reconstruction algorithm and give examples. Formula (11.1) appeared in [19].

Using (2.17) and (2.1) one gets

$$
I(k)=\frac{i k-A(0)+\int_{0}^{\infty} A_{1}(y) e^{i k y} d y}{1+\int_{0}^{\infty} e^{i k y} A(y) d y}, \quad A(y):=A(0, y), \quad A_{1}(y):=A_{x}(0, y) .
$$

The function

$$
f(k)=1+\int_{0}^{\infty} A(y) e^{i k y} d y=f_{0}(k) \frac{k}{k+i} \prod_{j=1}^{J} \frac{k-i k_{j}}{k+i k_{j}},
$$

where $f_{0}(k)$ is analytic in $\mathbb{C}_{+}, f_{0}(\infty)=1$ in $\mathbb{C}_{+}$, that is,

$$
f_{0}(k) \rightarrow 1 \text { as }|k| \rightarrow \infty, \quad \text { and } f_{0}(k) \neq 0, \quad \forall k \in \overline{\mathbb{C}}_{+}:=\{k: \operatorname{Im} k \geq 0\},
$$

and we assume that $k_{j} \neq 1$ without loss of generality: if $k_{j}=1$ for some $j$, then one can replace $k+i$ by $k+c i$, where $c>0$ and $c \neq k_{j}$ for all $j$.

Let us prove

Lemma 11.1 If $f(0) \neq 0$ and $q \in L_{1,1}\left(\mathbb{R}_{+}\right)$then

$$
f_{0}(k)=1+\int_{0}^{\infty} b_{0}(t) e^{i k t} d t:=1+\widetilde{b}_{0}, \quad b_{0} \in W^{1,1}\left(\mathbb{R}_{+}\right) .
$$

Here $W^{1,1}\left(\mathbb{R}_{+}\right)$is the Sobolev space of functions with the finite norm

$$
\left\|b_{0}\right\|_{W^{1,1}}:=\int_{0}^{\infty}\left(\left|b_{0}(t)\right|+\left|b_{0}^{\prime}(t)\right|\right) d t<\infty .
$$

Proof It is sufficient to prove that, for any $1 \leq j \leq J$, the function

$$
\frac{k+i k_{j}}{k-i k_{j}} f(k)=1+\int_{0}^{\infty} g_{j}(t) e^{i k t} d t, \quad g_{j} \in W^{1,1}\left(\mathbb{R}_{+}\right) .
$$

Since $\frac{k+i k_{j}}{k-i k_{j}}=1+\frac{2 i k_{j}}{k-i k_{j}}$, and since $A(y) \in W^{1,1}\left(\mathbb{R}_{+}\right)$provided that $q \in L_{1,1}\left(\mathbb{R}_{+}\right)$ (see (2.18), (2.19)), it is sufficient to check that

$$
\frac{f(k)}{k-i k_{j}}=\int_{0}^{\infty} g(t) e^{i k t} d t, \quad g \in W^{1,1}\left(\mathbb{R}_{+}\right)
$$


One has $f\left(i k_{j}\right)=0$, thus

$$
\begin{aligned}
\frac{f(k)}{k-i k_{j}} & =\frac{f(k)-f(i k)}{k-i k_{j}}=\int_{0}^{\infty} d y A(y) \frac{e^{i\left(k-i k_{j}\right) y}-1}{k-i k_{j}} e^{-k_{j} y} d y \\
& =\int_{0}^{\infty} A(y) e^{-k_{j} y} i \int_{0}^{y} e^{i\left(k-i k_{j}\right) s} d s=\int_{0}^{\infty} e^{i k s} h_{j}(s) d s
\end{aligned}
$$

where

$$
h_{j}(s):=i \int_{s}^{\infty} A(y) e^{-k_{j}(y-s)} d y=i \int_{0}^{\infty} A(t+s) e^{-k_{j} t} d t
$$

From (11.11) one obtains (11.9) since $A(y) \in W^{1,1}\left(\mathbb{R}_{+}\right)$.

Lemma 11.1 is proved.

Lemma 11.2 If $f(0)=0$ and $q \in L_{1,2}\left(\mathbb{R}_{+}\right)$, then (11.7) holds.

Proof The proof goes as above with one difference : if $f(0)=0$ then $k_{0}=0$ is present in formula (11.1) and in formulas (11.10) and (11.11) with $k_{0}=0$ one has

$$
h_{0}(s)=i \int_{0}^{\infty} A(t+s) d t
$$

Thus, using (2.18), one gets

$$
\begin{aligned}
& \int_{0}^{\infty}\left|h_{0}(s)\right| d s \leq c \int_{0}^{\infty} d s \int_{0}^{\infty} d t \int_{\frac{t+s}{2}}^{\infty}|q(u)| d u \\
& \quad=2 c \int_{0}^{\infty} d s \int_{\frac{s}{2}}^{\infty} d v \int_{v}^{\infty}|q(u)| d u \leq 2 c \int_{0}^{\infty} d s \int_{\frac{s}{2}}^{\infty}|q(u)| u d u \\
& \quad=4 c \int_{0}^{\infty} u^{2}|q(u)| d u<\infty \quad \text { if } \quad q \in L_{1,2}\left(\mathbb{R}_{+}\right),
\end{aligned}
$$

where $c>0$ is a constant. Similarly one checks that $h_{0}^{\prime}(s) \in L^{1}\left(\mathbb{R}_{+}\right)$if $q \in$ $L_{1,2}\left(\mathbb{R}_{+}\right)$.

Lemma 11.2 is proved.

Lemma 11.3 Formula (11.1) holds.

Proof Write

$$
\frac{1}{f(k)}=\frac{\frac{k+i}{k} \prod_{j=1}^{J} \frac{k+i k_{j}}{k-i k_{j}}}{f_{0}(k)}
$$

Clearly

$$
\frac{k+i}{k} \prod_{j=1}^{J} \frac{k+i k_{j}}{k-i k_{j}}=1+\sum_{j=0}^{J} \frac{c_{j}}{k-i k_{j}}, \quad k_{0}:=0, \quad k_{j}>0 .
$$

By the Wiener-Levy theorem $[8, \S 17]$, one has

$$
\frac{1}{f_{0}(k)}=1+\int_{0}^{\infty} b(t) e^{i k t} d t, \quad b(t) \in W^{1,1}\left(\mathbb{R}_{+}\right) .
$$

Actually, the Wiener-Levy theorem yields $b(t) \in L^{1}\left(\mathbb{R}_{+}\right)$.

However, since $b_{0} \in W^{1,1}\left(\mathbb{R}_{+}\right)$, one can prove that $b(t) \in W^{1,1}\left(\mathbb{R}_{+}\right)$.

Indeed, $\widetilde{b}$ and $\widetilde{b}_{0}$ are related by the equation:

$$
\left(1+\widetilde{b}_{0}\right)(1+\widetilde{b})=1, \quad \forall k \in \mathbb{R},
$$


which implies

$$
\widetilde{b}=-\widetilde{b}_{0}-\widetilde{b}_{0} \widetilde{b}
$$

or

$$
b(t)=-b_{0}(t)-\int_{0}^{t} b_{0}(t-s) b(s) d s:=-b_{0}-b_{0} * b,
$$

where $*$ is the convolution operation.

Since $b_{0}^{\prime} \in L^{1}\left(\mathbb{R}_{+}\right)$and $b \in L^{1}\left(\mathbb{R}_{+}\right)$the convolution $b_{0}^{\prime} * b \in L^{1}\left(\mathbb{R}_{+}\right)$. So, differentiating (11.19) one sees that $b^{\prime} \in L^{1}\left(\mathbb{R}_{+}\right)$, as claimed.

From (11.16), (11.15) and (11.4) one gets:

$$
I(k)=\left(i k-A(0)+\widetilde{A}_{1}\right)(1+\widetilde{b})\left(1+\sum_{j=0}^{J} \frac{c_{j}}{k-i k_{j}}\right)=i k+c+\sum_{j=0}^{J} \frac{a_{j}}{k-i k_{j}}+\widetilde{a},
$$

where $c$ is a constant defined in (11.24) below, the constants $a_{j}$ are defined in (11.25) and the function $\widetilde{a}$ is defined in (11.26). We will prove that $c=0$ (see (11.28)).

To derive (11.20), we have used the formula:

$$
i k \widetilde{b}=i k\left[\left.\frac{e^{i k t}}{i k} b(t)\right|_{0} ^{\infty}-\frac{1}{i k} \int_{0}^{\infty} e^{i k t} b^{\prime}(t) d t\right]=-b(0)-\widetilde{b}^{\prime},
$$

and made the following transformations:

$$
\begin{gathered}
I(k)=i k-A(0)-b(0)-\widetilde{b}^{\prime}+\widetilde{A}_{1}-A(0) \widetilde{b}+\widetilde{A}_{1} \widetilde{b} \sum_{j=0}^{J} \frac{c_{j} i k}{k-i k_{j}} \\
-\sum_{j=0}^{J} \frac{c_{j}[A(0)+b(0)]}{k-i k_{j}}+\sum_{j=0}^{J} \frac{\widetilde{g}(k)-\widetilde{g}\left(i k_{j}\right)}{k-i k_{j}} c_{j}+\sum_{j=0}^{J} \frac{\widetilde{g}\left(i k_{j}\right) c_{j}}{k-i k_{j}},
\end{gathered}
$$

where

$$
\widetilde{g}(k):=-\widetilde{b}^{\prime}+\widetilde{A}_{1}-A(0) \widetilde{b}+\widetilde{A}_{1} \widetilde{b}
$$

Comparing (11.22) and (11.20) one concludes that

$$
\begin{gathered}
c:=-A(0)-b(0)+i \sum_{j=0}^{J} c_{j}, \\
a_{j}:=-c_{j}\left[k_{j}+A(0)+b(0)-\widetilde{g}\left(i k_{j}\right)\right], \\
\widetilde{a}(k):=\widetilde{g}(k)+\sum_{j=0}^{J} \frac{\widetilde{g}(k)-\widetilde{g}\left(i k_{j}\right)}{k-i k_{j}} c_{j} .
\end{gathered}
$$

To complete the proof of Lemma 11.3 one has to prove that $c=0$, where $c$ is defined in (11.24). This is easily seen from the asymptotics of $I(k)$ as $k \rightarrow \infty$. Namely, one has, as in (11.21):

$$
\widetilde{A}(k)=-\frac{A(0)}{i k}-\frac{1}{i k} \widetilde{A}^{\prime}
$$

From (11.27) and (11.4) it follows that

$$
\begin{aligned}
I(k) & =\left(i k-A(0)+\widetilde{A}_{1}\right)\left[1-\frac{A(0)}{i k}+o\left(\frac{1}{k}\right)\right]^{-1} \\
& =\left(i k-A(0)+\widetilde{A}_{1}\right)\left(1+\frac{A(0)}{i k}+o\left(\frac{1}{k}\right)\right)=i k+o(1), \quad k \rightarrow+\infty
\end{aligned}
$$


From (11.28) and (11.20) it follows that $c=0$.

Lemma 11.3 is proved.

Lemma 11.4 One has $a_{j}=i r_{j}, r_{j}>0,1 \leq j \leq J$, and $r_{0}=0$ if $f(0) \neq 0$, and $r_{0}>0$ if $f(0)=0$.

Proof One has

$$
a_{j}=\operatorname{Res}_{k=i k_{j}} I(k)=\frac{f^{\prime}\left(0, i k_{j}\right)}{\dot{f}\left(i k_{j}\right)} .
$$

From (2.7) and (11.29) one gets:

$$
a_{j}=-\frac{c_{j}}{2 i k_{j}}=i \frac{c_{j}}{2 k_{j}}:=i r_{j}, \quad r_{j}:=\frac{c_{j}}{2 k_{j}}>0, \quad j>0
$$

If $j=0$, then

$$
a_{0}=\operatorname{Res}_{k=0} I(k):=\frac{f^{\prime}(0,0)}{\dot{f}(0)} .
$$

Here by $\operatorname{Res}_{k=0} I(k)$ we mean the right-hand side of (11.31) since $I(k)$ is, in general, not analytic in a disc centered at $k=0$, it is analytic in $\mathbb{C}_{+}$and, in general, cannot be continued analytically into $\mathbb{C}_{-}$. in $\overline{\mathbb{C}}_{+}$.

Let us assume $q(x) \in L_{1,2}\left(\mathbb{R}_{+}\right)$. In this case $f(k)$ is continuously differentiable

From the Wronskian formula

$$
\frac{f^{\prime}(0, k) f(-k)-f^{\prime}(0,-k) f(k)}{k}=2 i
$$

taking $k \rightarrow 0$, one gets

$$
f^{\prime}(0,0) \dot{f}(0)=-i .
$$

Therefore if $q \in L_{1,2}\left(\mathbb{R}_{+}\right)$and $f(0)=0$, then $\dot{f}(0) \neq 0$ and $f^{\prime}(0,0) \neq 0$. One can prove $\left[14\right.$, pp.188-190], that if $q \in L_{1,1}\left(\mathbb{R}_{+}\right)$, then $\frac{k}{f(k)}$ is bounded as $k \rightarrow 0$, $k \in \mathbb{C}_{+}$.

From (11.31) and (11.33) it follows that

$$
a_{0}=-\frac{i}{[\dot{f}(0)]^{2}}=i r_{0}, \quad r_{0}:=-\frac{1}{[\dot{f}(0)]^{2}} \text {. }
$$

From (2.17) one gets:

$$
\dot{f}(0)=i \int_{0}^{\infty} A(y) y d y
$$

Since $A(y)$ is a real-valued function if $q(x)$ is real-valued (this follows from the integral equation (5.62), formula (11.35) shows that

$$
[\dot{f}(0)]^{2}<0
$$

and (11.34) implies

$$
r_{0}>0
$$

Lemma 11.4 is proved. 
One may be interested in the properties of function $a(t)$ in (11.1). These can be obtained from $(11.26),(11.16)$ and $(11.7)$ as in the proof of Lemmas 11.1 and 11.2 .

In particular (11.2) and (11.3) can be obtained.

Note that even if $q(x) \not \equiv 0$ is compactly supported, one cannot claim that $a(t)$ is compactly supported.

This can be proved as follows.

Assume for simplicity that $J=0$ and $f(0) \neq 0$. Then if $a(t)$ is compactly supported then $I(k)$ is an entire function of exponential type. It is proved in $[17$, p.278] that if $q(x) \not \equiv 0$ is compactly supported, $q \in L^{1}\left(\mathbb{R}_{+}\right)$, then $f(k)$ has infinitely many zeros in $\mathbb{C}$. The function $f^{\prime}(0, z) \neq 0$ if $f(z)=0$. Indeed, if $f(z)=0$ and $f^{\prime}(0, z)=0$ then $f(x, z) \equiv 0$ by the uniqueness of the solution of the Cauchy problem for equation (1.1) with $k=z$. Since $f(x, z) \not \equiv 0$ (see (1.3)), one has a contradiction, which proves that $f^{\prime}(0, z) \neq 0$ if $f(z)=0$. Thus $I(k)$ cannot be an entire function if $q(x) \not \equiv 0, q(x) \in L^{1}\left(\mathbb{R}_{+}\right)$and $q(x)$ is compactly supported.

Let us consider the following question:

What are the potentials for which $a(t)=0$ in (11.1)?

In other words, suppose

$$
I(k)=i k+\sum_{j=0}^{J} \frac{i r_{j}}{k-i k_{j}}
$$

find $q(x)$ corresponding to $I$-function (11.38), and describe the decay properties of $q(x)$ as $x \rightarrow+\infty$.

We now show two ways of doing this.

By definition

$$
f^{\prime}(0, k)=I(k) f(k), \quad f^{\prime}(0,-k)=I(-k) f(-k), \quad k \in \mathbb{R} .
$$

Using (11.39) and (2.23) one gets

$$
[I(k)-I(-k)] f(k) f(-k)=2 i k,
$$

or

$$
f(k) f(-k)=\frac{k}{\operatorname{ImI}(k)}, \quad \forall k \in \mathbb{R} .
$$

By (2.5), (2.6) and (11.30) one can write (see [19]) the spectral function corresponding to the $I$-function $(11.38)(\sqrt{\lambda}=k)$ :

$$
d \rho(\lambda)= \begin{cases}\frac{\operatorname{Im} I(\lambda)}{\pi} d \lambda, & \lambda \geq 0 \\ \sum_{j=1}^{J} 2 k_{j} r_{j} \delta\left(\lambda+k_{j}^{2}\right) d \lambda, & \lambda<0\end{cases}
$$

where $\delta(\lambda)$ is the delta-function.

Knowing $d \rho(\lambda)$ one can recover $q(x)$ algorithmically by the scheme (5.26).

Consider an example. Suppose $f(0) \neq 0, \quad J=1$,

$$
I(k)=i k+\frac{i r_{1}}{k-i k_{1}}=i k+\frac{i r_{1}\left(k+i k_{1}\right)}{k^{2}+k_{1}^{2}}=i\left(k+\frac{r_{1} k}{k^{2}+k_{1}^{2}}\right)-\frac{r_{1} k_{1}}{k^{2}+k_{1}^{2}} \text {. }
$$

Then (11.41) yields:

$$
d \rho(\lambda)= \begin{cases}\frac{d \lambda}{\pi}\left(\sqrt{\lambda}+\frac{r_{1} \sqrt{\lambda}}{\lambda+k_{1}^{2}}\right), & \lambda>0 \\ 2 k_{1} r_{1} \delta\left(\lambda+k_{1}^{2}\right) d \lambda, & \lambda<0\end{cases}
$$


Thus (5.27) yields:

$$
L(x, y)=\frac{1}{\pi} \int_{0}^{\infty} d \lambda \frac{r_{1} \sqrt{\lambda}}{\lambda+k_{1}^{2}} \frac{\sin \sqrt{\lambda} x}{\sqrt{\lambda}} \frac{\sin \sqrt{\lambda} y}{\sqrt{\lambda}}+2 k_{1} r_{1} \frac{\operatorname{sh}\left(k_{1} x\right)}{k_{1}} \frac{\operatorname{sh}\left(k_{1} y\right)}{k_{1}},
$$

and, setting $\lambda=k^{2}$ and taking for simplicity $2 k_{1} r_{1}=1$, one finds:

$$
\begin{aligned}
L_{0}(x, y) & :=\frac{2 r_{1}}{\pi} \int_{0}^{\infty} \frac{d k k^{2}}{k^{2}+k_{1}^{2}} \frac{\sin (k x) \sin (k y)}{k^{2}} \\
& =\frac{2 r_{1}}{\pi} \int_{0}^{\infty} \frac{d k \sin (k x) \sin (k y)}{k^{2}+k_{1}^{2}} \\
& =\frac{r_{1}}{\pi} \int_{0}^{\infty} \frac{d k[\cos k(x-y)-\cos k(x+y)]}{k^{2}+k_{1}^{2}} \\
& =\frac{r_{1}}{2 k_{1}}\left(e^{-k_{1}|x-y|}-e^{-k_{1}(x+y)}\right), \quad k_{1}>0,
\end{aligned}
$$

where the known formula was used:

$$
\frac{1}{\pi} \int_{0}^{\infty} \frac{\cos k x}{k^{2}+a^{2}} d k=\frac{1}{2 a} e^{-a|x|}, \quad a>0, \quad x \in \mathbb{R} .
$$

Thus

$$
L(x, y)=\frac{r_{1}}{2 k_{1}}\left[e^{-k_{1}|x-y|}-e^{-k_{1}(x+y)}\right]+\frac{\operatorname{sh}\left(k_{1} x\right)}{k_{1}} \frac{\operatorname{sh}\left(k_{1} y\right)}{k_{1}} .
$$
kernel:

Equation (5.30) with kernel (11.47) is not an integral equation with degenerate

$$
\begin{aligned}
K(x, y) & +\int_{0}^{x} K(x, t)\left[\frac{e^{-k_{1}|t-y|}-e^{-k_{1}(t+y)}}{2 k_{1} / r_{1}}+\frac{\operatorname{sh}\left(k_{1} t\right)}{k_{1}} \frac{\operatorname{sh}\left(k_{1} y\right)}{k_{1}}\right] d t \\
& =-\frac{e^{-k_{1}|x-y|}-e^{-k_{1}(x+y)}}{2 k_{1} / r_{1}}-\frac{\operatorname{sh}\left(k_{1} x\right)}{k_{1}} \frac{\operatorname{sh}\left(k_{1} y\right)}{k_{1}} .
\end{aligned}
$$

This equation can be solved analytically [34], but the solution requires space to present. Therefore we do not give the theory developed in [34] but give another approach to a study of the properties of $q(x)$ given $I(k)$ of the form (11.42). This approach is based on the theory of the Riemann problem [6].

Equations (11.40) and (11.42) imply

$$
f(k) f(-k)=\frac{k^{2}+k_{1}^{2}}{k^{2}+\nu_{1}^{2}}, \quad \nu_{1}^{2}:=k_{1}^{2}+r_{1} .
$$

The function

$$
f_{0}(k):=f(k) \frac{k+i k_{1}}{k-i k_{1}} \neq 0 \quad \text { in } \quad \mathbb{C}_{+} .
$$

Write (11.49) as

$$
f_{0}(k) \frac{k-i k_{1}}{k+i k_{1}} f_{0}(-k) \frac{k+i k_{1}}{k-i k_{1}}=\frac{k^{2}+k_{1}^{2}}{k^{2}+\nu_{1}^{2}} .
$$

Thus

$$
f_{0}(k)=\frac{k^{2}+k_{1}^{2}}{k^{2}+\nu_{1}^{2}} h \quad h(k):=\frac{1}{f_{0}(-k)} .
$$

The function $f_{0}(-k) \neq 0$ in $\mathbb{C}_{-}, f_{0}(\infty)=1$ in $\mathbb{C}_{-}$, so $h:=\frac{1}{f_{0}(-k)}$ is analytic in $\mathbb{C}_{-}$. 
Consider (11.51) as a Riemann problem. One has

$$
i n d_{\mathbb{R}} \frac{k^{2}+k_{1}^{2}}{k^{2}+\nu_{1}^{2}}:=\frac{1}{2 \pi i} \int_{-\infty}^{\infty} d \ln \frac{k^{2}+k_{1}^{2}}{k^{2}+\nu_{1}^{2}}=0 .
$$

Therefore (see [6]) problem (11.51) is uniquely solvable. Its solution is:

$$
f_{0}(k)=\frac{k+i k_{1}}{k+i \nu_{1}}, \quad h(k)=\frac{k-i \nu_{1}}{k-i k_{1}},
$$

as one can check.

Thus, by (11.50),

$$
f(k)=\frac{k-i k_{1}}{k+i \nu_{1}}
$$

The corresponding $S$-matrix is:

$$
S(k)=\frac{f(-k)}{f(k)}=\frac{\left(k+i k_{1}\right)\left(k+i \nu_{1}\right)}{\left(k-i k_{1}\right)\left(k-i \nu_{1}\right)}
$$

Thus

$$
\begin{gathered}
F_{S}(x):=\frac{1}{2 \pi} \int_{-\infty}^{\infty}[1-S(k)] e^{i k x} d k=O\left(e^{-k_{1} x}\right) \quad \text { for } \quad x>0, \\
F_{d}(x)=s_{1} e^{-k_{1} x}
\end{gathered}
$$

and

$$
F(x)=F_{S}(x)+F_{d}(x)=O\left(e^{-k_{1} x}\right) .
$$

Equation (5.50) implies $A(x, x)=O\left(e^{-2 k_{1} x}\right)$, so

$$
q(x)=O\left(e^{-2 k_{1} x}\right), \quad x \rightarrow+\infty .
$$

Thus, if $f(0) \neq 0$ and $a(t)=0$ then $q(x)$ decays exponentially at the rate determined by the number $k_{1}, k_{1}=\min _{1 \leq j \leq J} k_{j}$.

If $f(0)=0, J=0$, and $a(t)=0$, then

$$
\begin{gathered}
I(k)=i k+\frac{i r_{0}}{k}, \\
f(k) f(-k)=\frac{k^{2}}{k^{2}+r_{0}}, \quad r_{0}>0 .
\end{gathered}
$$

Let $f_{0}(k)=\frac{(k+i) f(k)}{k}$. Then equation (11.60) implies:

$$
f_{0}(k) f_{0}(-k)=\frac{k^{2}+1}{k^{2}+\nu_{0}^{2}}, \quad \nu_{0}^{2}:=r_{0},
$$

and $f_{0}(k) \neq 0$ in $\mathbb{C}_{+}$.

Thus, since $i n d_{\mathbb{R}} \frac{k^{2}+1}{k^{2}+\nu_{0}^{2}}=0, f_{0}(k)$ is uniquely determined by the Riemann problem (11.61).

One has:

and

$$
f_{0}(k)=\frac{k+i}{k+i \nu_{0}}, \quad f_{0}(-k)=\frac{k-i}{k-i \nu_{0}},
$$

$$
\begin{gathered}
f(k)=\frac{k}{k+i \nu_{0}}, \quad S(k)=\frac{f(-k)}{f(k)}=\frac{k+i \nu_{0}}{k-i \nu_{0}}, \\
F_{S}(x)=\frac{1}{2 \pi} \int_{-\infty}^{\infty}\left(1-\frac{k+i \nu_{0}}{k-i \nu_{0}}\right) e^{i k x} d k=\frac{-2 i \nu_{0}}{2 \pi} \int_{-\infty}^{\infty} \frac{e^{i k x} d k}{k-i \nu_{0}}=2 \nu_{0} e^{-\nu_{0} x}, \quad x>0
\end{gathered}
$$


and $F_{d}(x)=0$.

So one gets:

$$
F(x)=F_{S}(x)=2 \nu_{0} e^{-\nu_{0} x}, \quad x>0 .
$$

Equation (5.50) yields:

$$
A(x, y)+2 \nu_{0} \int_{x}^{\infty} A(x, t) e^{-\nu_{0}(t+y)} d t=-2 \nu_{0} e^{-\nu_{0}(x+y)}, \quad y \geq x \geq 0 .
$$

Solving (11.64) yields:

$$
A(x, y)=-2 \nu_{0} e^{-\nu_{0}(x+y)} \frac{1}{1+e^{-2 \nu_{0} x}} .
$$

The corresponding potential (5.51) is

$$
q(x)=O\left(e^{-2 \nu_{0} x}\right), \quad x \rightarrow \infty .
$$

If $q(x)=O\left(e^{-k x}\right), k>0$, then $a(t)$ in (11.1) decays exponentially. Indeed, in this case $b^{\prime}(t), A_{1}(y), b(t), A_{1} * b$ decay expenentially, so, by (11.23), $g(t)$ decays exponentially, and, by (11.26), the function $\frac{\widetilde{g}(k)-\widetilde{g}\left(i k_{j}\right)}{k-i k_{j}}:=\widetilde{h}$ with $h(t)$ decaying exponentially. We leave the details to the reader.

\section{Algorithms for finding $q(x)$ from $I(k)$}

One algorithm, discussed in section 11, is based on finding the spectral function $\rho(\lambda)$ from $I(k)$ by formula (11.41) and then finding $q(x)$ by the method (5.26).

The second algorithm is based on finding the scattering data (2.10) and then finding $q(x)$ by the method (5.49).

In both cases one has to find $k_{j}, 1 \leq j \leq J$, and the number $J$. In the second method one has to find $f(k)$ and $s_{j}$ also, and $S(k)=\frac{f(-k)}{f(k)}$.

If $k_{j}$ and $f(k)$ are found then $s_{j}$ can be found from (2.12). Indeed, by (11.1)

$$
i r_{j}:=\operatorname{Res}_{k=i k_{j}} I(k)=\frac{f^{\prime}\left(0, i k_{j}\right)}{\dot{f}\left(i k_{j}\right)} .
$$

From (12.1) and (2.12) one finds

$$
s_{j}=-\frac{2 i k_{j}}{i r_{j}\left[\dot{f}\left(i k_{j}\right)\right]^{2}}=-\frac{2 k_{j}}{r_{j}\left[\dot{f}\left(i k_{j}\right)\right]^{2}} .
$$

If $k_{j}$ are found, then one can find $f(k)$ from $I(k)$ as follows. Since $f^{\prime}(0, k)=$ $f(k) I(k)$, equation (2.23) implies equation (11.40):

$$
f(k) f(-k)=\frac{k}{\operatorname{ImI}(k)} .
$$

Define

$$
w(k):=\prod_{j=1}^{J} \frac{k-i k_{j}}{k+i k_{j}} \quad \text { if } \quad I(0)<\infty, \quad f(0) \neq 0,
$$

and

$$
w(k):=\frac{k}{k+i} \prod_{j=1}^{J} \frac{k-i k_{j}}{k+i k_{j}} \quad \text { if } \quad I(0)=\infty, \quad f(0)=0 .
$$

One has $I(0)<\infty$ if $f(0) \neq 0$ and $I(0)=\infty$ if $f(0)=0$. Note that if $q \in L_{1,2}\left(\mathbb{R}_{+}\right)$and $f(0)=0$ then $f^{\prime}(0,0) \neq 0$ and $\dot{f}(0) \neq 0$. 
Define

$$
h(k):=\frac{f(k)}{w(k)} .
$$

Then $h(k)$ is analytic in $\mathbb{C}_{+}, h(k) \neq 0$ in $\overline{\mathbb{C}}_{+}$, and $h(\infty)=1$ in $\overline{\mathbb{C}}_{+}$, while $h(-k)$ has similar properties in $\mathbb{C}_{-}$. Denote $\frac{1}{h(-k)}:=h_{-}(k)$. This function is analytic in $\mathbb{C}_{-}, \quad h_{-}(k) \neq 0$ in $\overline{\mathbb{C}}_{-}$and $h_{-}(\infty)=1$ in $\bar{C}_{-}$. Denote $h(k):=h_{+}(k)$.

Write (12.3) as the Riemann problem:

$$
h_{+}(k)=g(k) h_{-}(k),
$$

where

$$
g(k)=\frac{k}{\operatorname{ImI}(k)} \quad \text { if } \quad I(0)<\infty
$$

and

$$
g(k)=\frac{k}{\operatorname{Im} I(k)} \frac{k^{2}+1}{k^{2}} \text { if } I(0)=\infty .
$$

We claim that the function $g(k)$ is positive for all $k>0$, bounded in a neighborhood of $k=0$ and has a finite limit at $k=0$ even if $I(0)=0$. Only the case $I(0)=0$ requires a comment. If $I(0)=0$, then $f^{\prime}(0,0)=0, \dot{f}^{\prime}(0,0) \neq 0, f(0) \neq 0$, and one can see from (12.3) that the function $\frac{k}{\operatorname{ImI} I(k)}$ is bounded. Thus, the claim is verified.

The Riemann problem (12.7) can be solved analytically: $\ln h_{+}(k)-\ln h_{-}(k)=$ $\ln g(k)$ and since $h_{+}(k)$ and $h_{-}(k)$ do not vanish in $\overline{\mathbb{C}}_{+}$and $\overline{\mathbb{C}}_{-}$respectively, $\ln h_{+}(k)$ and $\ln h_{-}(k)$ are analytic in $\mathbb{C}_{+}$and $\mathbb{C}_{-}$respectively. Therefore

$$
\begin{gathered}
h(k)=\exp \left(\frac{1}{2 \pi i} \int_{-\infty}^{\infty} \frac{\ln g(t)}{t-k} d t\right), \\
h(k)=h_{+}(k) \text { if } \operatorname{Im} k>0, \quad h(k)=h_{-}(k) \text { if } \operatorname{Im} k<0,
\end{gathered}
$$

and

$$
f(k)=w(k) h(k), \quad \operatorname{Im} k \geq 0 .
$$

Finally, let us explain how to find $k_{j}$ and $J$ given $I(k)$.

From (11.1) it follows that

$$
\frac{1}{2 \pi} \int_{-\infty}^{\infty}(I(k)-i k) e^{-i k t} d k=-\sum_{j=1}^{J} r_{j} e^{k_{j} t}-\frac{r_{0}}{2} \quad \text { for } \quad t<0 .
$$

Taking $t \rightarrow-\infty$ in (12.13) one can find step by step the numbers $r_{0}, k_{1}, r_{1}$, $k_{2}, r_{2} \ldots, r_{J}, k_{J}$. If $I(0)<\infty$, then $r_{0}=0$.

\section{Remarks.}

13.1 Representation of the products of the solution to (1.1). In this subsection we follow [12]. Consider equation (1.1) with $q=q_{j}, j=1,2$. The function $u(x, y):=\varphi_{1}(x, k) \varphi_{2}(y, k)$ where $\varphi_{j}, j=1,2$, satisfy the first two conditions (1.4), solves the problem

$$
\begin{gathered}
{\left[\frac{\partial^{2}}{\partial x^{2}}-q_{1}(x)\right] u(x, y)=\left[\frac{\partial^{2}}{\partial y^{2}}-q_{2}(y)\right] u(x, y)} \\
u(0, y)=0, \quad u_{x}(0, y)=\varphi_{2}(y, k) \\
u(x, 0)=0, \quad u_{y}(x, 0)=\varphi_{1}(x, k)
\end{gathered}
$$


Let us write (13.1) as

$$
\left(\frac{\partial^{2}}{\partial x^{2}}-\frac{\partial^{2}}{\partial y^{2}}\right) u(x, y)=\left[q_{1}(x)-q_{2}(y)\right] u(x, y)
$$

and use the known D'Alembert's formula to solve (13.3)-(13.4):

$$
u(x, y)=\frac{1}{2} \int_{D_{x y}}\left[q_{1}(s)-q_{2}(t)\right] u(s, t) d s d t+\frac{1}{2} \int_{x-y}^{x+y} \varphi_{1}(s) d s,
$$

where $D_{x y}$ is the triangle $0<t<y, x-y+t<s<x+y-t$.

Function (13.5) satifies (13.3) and (13.1). Equation (13.5) is uniquely solvable by iterations:

Note that

$$
\begin{gathered}
u(x, y)=\sum_{m=0}^{\infty} u_{m}(x, y), \quad u_{0}(x, y):=\frac{1}{2} \int_{x-y}^{x+y} \varphi_{1}(s, x) d s \\
u_{m+1}(x, y)=\frac{1}{2} \int_{D_{x y}}\left[q_{1}(s)-q_{2}(t)\right] u_{m}(s, t) d s d t
\end{gathered}
$$

$$
u_{m}(x, y)=\frac{1}{2} \int_{x-y}^{x+y} w_{m}(x, y, s) \varphi_{1}(s) d s .
$$

If $m=0$ this is clear from (13.6). If it is true for some $m>0$, then it is true for $m+1$ :

$$
\begin{aligned}
u_{m+1}(x, y) & =\frac{1}{2} \int_{0}^{y} d t \int_{x-y+t}^{x+y-t} d s\left[q_{1}(s)-q_{2}(t)\right] \frac{1}{2} \int_{s-t}^{s+t} w_{m}(s, t, \sigma) \varphi_{1}(\sigma) d \sigma \\
& =\frac{1}{2} \int_{0}^{y} d t \int_{x-y}^{x+y} d \sigma \varphi_{1}(\sigma) \widetilde{w}_{m}(x, y, t, \sigma) \\
& =\frac{1}{2} \int_{x-y}^{x+y} d \sigma \varphi_{1}(\sigma) w_{m+1}(x, y, \sigma)
\end{aligned}
$$

where $\widetilde{w}_{m}$ and $w_{m+1}$ are some functions.

Thus, by induction, one gets (13.8) for all $m$, and (13.6) implies

$$
u(x, y)=\frac{1}{2} \int_{x-y}^{x+y} w(x, y, s) \varphi_{1}(s) d s,
$$

where

$$
w(x, y, s):=\sum_{m=0}^{\infty} w_{m}(x, y, s) .
$$

To satisfy (13.2) one has to satisfy the equations:

$$
\begin{aligned}
0= & \int_{-y}^{y} w(0, y, s) \varphi_{1}(s, k) d s \\
\varphi_{2}(y, k)= & \frac{1}{2}\left[w(0, y, y) \varphi_{1}(y)-w(0, y,-y) \varphi_{1}(-y)\right] \\
& +\frac{1}{2} \int_{-y}^{y} w_{x}(0, y, s) \varphi_{1}(s) d s .
\end{aligned}
$$

Formula (13.10) yields

$$
\varphi_{1}(x, k) \varphi_{2}(y, k)=\frac{1}{2} \int_{x-y}^{x+y} w(x, y, s) \varphi_{1}(s, k) d s .
$$


If $x=y$, then

$$
\varphi_{1}(x, k) \varphi_{2}(x, k)=\frac{1}{2} \int_{0}^{2 x} w(x, x, s) \varphi_{1}(s, k) d s .
$$

Therefore, if

$$
\int_{0}^{a} h(x) \varphi_{1}(x, k) \varphi_{2}(x, k) d x=0 \quad \forall k>0
$$

then

$$
\begin{aligned}
0 & =\int_{0}^{a} h(x) \int_{0}^{2 x} w(x, x, s) \varphi_{1}(s, k) d s d x \\
& =\int_{0}^{2 a} d s \varphi_{1}(s, k) \int_{\frac{s}{2}}^{a} d x h(x) w(x, x, s) \quad \forall k>0 .
\end{aligned}
$$

Since the set $\left\{\varphi_{1}(s, k)\right\}_{\forall k>0}$ is complete in $L^{2}(0,2 a)$, it follows that $0=\int_{\frac{s}{2}}^{a} d x h(x) w(x, x, s)$ for all $s \in[0,2 a]$. Differentiate with respect to $s$ and get

$$
w\left(\frac{s}{2}, \frac{s}{2}, s\right) \frac{1}{2} h\left(\frac{s}{2}\right)-\int_{\frac{s}{2}}^{a} d s h(x) w_{s}(x, x, s)=0 .
$$

From Volterra equation (13.15) it follows $h(x)=0$ if the kernel $w_{s}(x, x, s) w^{-1}\left(\frac{s}{2}, \frac{s}{2}, s\right):=$ $t(x, s)$ is summable. From the definition (13.11) of $w$ it follows that if $\int_{0}^{b}|q(x)| d x<$ $\infty \forall b>0$, then $w_{s}(x, y, s)$ is summable. The function $w(x, y, s)$ has $m$ summable derivatives with respect to $x, y$ and $s$ if $q(x)$ has $m-1$ summable derivatives. Thus one can derive from (13.15) that $h(x)=0$ if $w\left(\frac{s}{2}, \frac{s}{2}, s\right)>0$ for all $s \in[0,2 a]$.

If the boundary conditions at $x=0$ are different, for example, $\varphi_{j}^{\prime}(0, k)-$ $h_{0} \varphi_{j}(0, k)=0, j=1,2$, then conditions

$$
u_{x}-\left.h_{0} u\right|_{x=0}=0, \quad u_{y}-\left.h_{0} u\right|_{y=0}=0, \quad h_{0}=\text { const }>0
$$

replace the first conditions (13.2) and (13.3). One can normalize $\varphi_{j}(x, k)$ by setting

$$
\varphi_{j}(0, k)=1 \text {. }
$$

Then

$$
\begin{gathered}
\varphi_{j}^{\prime}(0, k)=h_{0}, \\
u(0, y)=\varphi_{2}(y, k), \quad u(x, 0)=\varphi_{1}(x, k), \\
u_{x}(0, k)=h_{0} \varphi_{2}(y, k), \quad u_{y}(x, 0)=h_{0} \varphi_{1}(x, k),
\end{gathered}
$$

and (13.5) is replaced by

$$
\begin{aligned}
u(x, y) & =\frac{1}{2} \int_{D_{x y}}\left[q_{1}(s)-q_{2}(t)\right] u(s, t) d s d t \\
& +\frac{1}{2} \int_{x-y}^{x+y} h_{0} \varphi_{1}(s, k) d s+\frac{1}{2}\left[\varphi_{1}(x+y, k)+\varphi_{1}(x-y, k)\right] .
\end{aligned}
$$

Note that

$$
\frac{1}{2}\left[\varphi_{1}(x+y)+\varphi_{1}(x-y)\right]=\frac{1}{2} \frac{\partial}{\partial y} \int_{x-y}^{x+y} \varphi_{1}(s) d s=\frac{1}{2} \int_{x-y}^{x+y} \varphi_{s}(s) d s .
$$

Equation (13.21) is uniquely solvable by iterations, as above, and its solution is given by the first formula (13.6) with

$$
u_{0}(x, y)=\frac{h_{0}}{2} \int_{x-y}^{x+y} \varphi_{1}(s, k) d s+\frac{1}{2} \int_{x-y}^{x+y} \varphi_{1 s}(s, k) d s .
$$


The rest of the argument is as above: one proves existence and uniqueness of the solution to equation (13.21) and the analog of formula (13.10):

$$
u(x, y)=\frac{1}{2} \int_{x-y}^{x+y} w(x, y, s) \Phi(s) d s, \quad \Phi:=h_{0} \varphi_{1}(s, k)+\varphi_{1 s}(s, k) .
$$

Thus

$$
u(x, x)=\varphi_{1}(x, k) \varphi_{2}(x, k)=\int_{0}^{2 x} t(x, s)\left[h_{0} \varphi_{1}(s, k)+\varphi_{1 s}(s, k)\right] d s,
$$

where

and $t(x, s)$ is summable.

$$
t(x, s):=\frac{1}{2} w(x, x, s)
$$

Thus, as before, completeness of the set of products $\left\{\varphi_{1}(x, k) \varphi_{2}(x, k)\right\}_{\forall k>0}$ can be studied.

13.2 Characterization of Weyl's solutions. The standard definition of Weyl's solution to (1.1) is given by (2.2).

In $[15]$ it is proved that

$$
W(x, k)=e^{i k x}(1+o(1)) \quad \text { as } \quad|k| \rightarrow \infty, \quad|x| \leq b, \quad k^{2} \in \Delta,
$$

where $\Delta:=\{\lambda:|\operatorname{Im} \lambda|>\varepsilon, \operatorname{dist}(\lambda, S)>\varepsilon\}$,

$$
S:=\mathbb{R} \cup\left[i \gamma_{-}, i \gamma_{+}\right], \quad \gamma_{ \pm}:=\inf _{\substack{u \in H^{1}\left(\mathbb{R}_{ \pm}\right) \\ u(0)=0}} \pm \int_{0}^{ \pm \infty}\left[u^{\prime 2}+q|u|^{2}\right] d x .
$$

The relation (13.27) gives a definition of the Weyl solution by its behavior on compact sets in the $x$-space as $|k| \rightarrow \infty$, as opposite to (2.2), where $k$ is fixed, and $x \rightarrow \infty$. For multidimensional Schrödinger equation similar definition was proposed in $[17$, p.356, problem 8].

We want to derive (13.27) for potentials in $L_{1,1}\left(\mathbb{R}_{+}\right)$and for $k>0, k \rightarrow+\infty$.

The idea is simple. For any $q=\bar{q} \in L_{l o c}^{1}\left(\mathbb{R}_{+}\right)$, one can construct $\varphi(x, k)$ and $\psi(x, k)$, the solutions to (1.1) and (1.4), for any $|x| \leq b$, where $b>0$ is an arbitrary large fixed number, by solving the Volterra equations

$$
\begin{aligned}
& \varphi(x, k)=\frac{\sin (k x)}{k}+\int_{0}^{x} \frac{\sin [k(x-y)]}{k} q(y) \varphi(y, k) d y \\
& \psi(x, k)=\cos (k x)+\int_{0}^{x} \frac{\sin [k(x-y)]}{k} q(y) \psi(y, k) d y .
\end{aligned}
$$

One can also write an equation for the Weyl solution $W$ :

$$
W(x, k)=\cos (k x)+m(k) \frac{\sin (k x)}{k}+\int_{0}^{x} \frac{\sin [k(x-y)]}{k} q(y) W(y, k) d y .
$$

This equation is uniquely solvable by iterations for $|x| \leq b$.

It is known that

$$
m(k)=i k+o(1), \quad|k| \rightarrow \infty, \quad \operatorname{Im} k>\varepsilon|\operatorname{Re} k|, \quad \varepsilon>0 .
$$

For $q \in L_{1,1}\left(\mathbb{R}_{+}\right)$the above formula holds when $k>0, k \rightarrow+\infty$. From (13.31) and (13.32) one gets, assuming $k>0$,

$$
W(x, k)=e^{i k x}\left(1+O\left(\frac{1}{k}\right)\right)+\int_{0}^{x} \frac{\sin [k(x-y)]}{k} q(y) W(y, k) d y .
$$


Solving (13.33) by iterations yields (13.27) for $k>0, k \rightarrow+\infty$. For $q \in L_{1,1}\left(\mathbb{R}_{+}\right)$ the Weyl solution is the Jost solution. Therefore the above result for $k>0, k \rightarrow+\infty$ is just the standard asymptotics for the Jost solution. It would be of interest to generalize the above approach to the case of complex $k$ in the region (13.27).

One can look for an asymptotic representation of the solution to (1.1) for large $|k|, \operatorname{Im} k>\varepsilon|\operatorname{Re} k|, \varepsilon>0$, of the following form:

$$
u(x, k)=e^{i k x+\int_{0}^{x} \sigma(t, k) d t}
$$

where

$$
\sigma^{\prime}+2 i k \sigma+\sigma^{2}-q(x)=0, \quad \sigma=\frac{q(x)}{2 i k}+o\left(\frac{1}{k}\right), \quad|k| \rightarrow \infty .
$$

From (13.34) one finds, assuming $q(x)$ continuous at $x=0$,

$$
\frac{u^{\prime}(0, k)}{u(0, k)}=i k+\frac{q(0)}{2 i k}+o\left(\frac{1}{k}\right), \quad|k| \rightarrow \infty, \quad k \in \mathbb{C}_{+} .
$$

If $q(x)$ has $n$ derivatives, more terms of the asymptotics can be written (see [14, p.55]).

13.3 Representation of the Weyl function via the Green function. The Green function of the Dirichlet operator $L_{q}=-\frac{d^{2}}{d x^{2}}+q(x)$ in $L^{2}\left(\mathbb{R}_{+}\right)$can be written as:

$$
G(x, y, z)=\varphi(y, \sqrt{z}) W(x, \sqrt{z}), \quad x \geq y
$$

where $\varphi(x, k), k:=\sqrt{z}$, solves (1.1) and satisfies the first two conditions (1.4), and $W(x, \sqrt{z})$ is the Weyl solution $(2.2)$, which satisfies the conditions:

$$
W(0, \sqrt{z})=1, \quad W^{\prime}(0, \sqrt{z})=m(\sqrt{z}) .
$$

From (1.4), (13.37) and (13.38) it follows that:

$$
\left.\frac{\partial^{2} G(x, y, k)}{\partial x \partial y}\right|_{x=y=0}=m(k) \text {. }
$$

If $q \in L_{1,1}\left(\mathbb{R}_{+}\right)$then $W(x, k)=\frac{f(x, k)}{f(k)}$, where $f(x, k)$ is the Jost solution (1.3), $k \in \mathbb{C}_{+}$. Note that (13.17) and (13.38) imply:

$$
\frac{1}{\pi} \operatorname{Im} G(x, y, \lambda+i 0)=\frac{\varphi(x, \sqrt{\lambda}) \varphi(y, \sqrt{\lambda})}{\pi} \operatorname{Im} m(\sqrt{\lambda+i 0})
$$

and

$$
G(x, y, z)=\int_{-\infty}^{\infty} \frac{\theta(x, y, t)}{t-z} d \rho(t), \quad \theta(x, y, t)=\varphi(x, \sqrt{t}) \varphi(y, \sqrt{t}) .
$$

Thus

$$
\frac{1}{\pi} \operatorname{Im} G(x, y, t+i 0) d t=\theta(x, y, t) d \rho(t) .
$$

From (13.42) and (13.40) one gets, assuming $\rho(-\infty)=0$,

$$
\rho(t)=\frac{1}{\pi} \int_{-\infty}^{t} \operatorname{Im} m(\sqrt{\lambda+i 0}) d \lambda .
$$


If $\lambda<0$ then $\operatorname{Im} m(\sqrt{\lambda+i 0})=0$ except at the points $\lambda=-k_{j}^{2}$ at which $f\left(i k_{j}\right)=0$, so that $m\left(\sqrt{-k_{j}^{2}+i 0}\right)=\infty$. Thus, if $t$ and $a$ are continuity points of $\rho(t)$, then

$$
\rho(t)-\rho(a)=\frac{1}{\pi} \int_{a}^{t} \operatorname{Im} m(\sqrt{\lambda+i 0}) d \lambda, \quad a \geq 0 .
$$

Let us recall the Stieltjes inversion formula:

If $z=\sigma+i \tau, \tau>0, \rho(t)$ is a function of bounded variation on $\mathbb{R}$,

$$
\varphi(z):=\int_{-\infty}^{\infty} \frac{d \rho(t)}{t-z}
$$

and if $a$ and $b$ are continuity points of $\rho(t)$, then

$$
\frac{1}{\pi} \int_{a}^{b} \operatorname{Im} \varphi(\lambda+i 0) d \lambda=\rho(b)-\rho(a) .
$$

Therefore (13.44) implies

$$
m(\sqrt{z})=\int_{-\infty}^{\infty} \frac{d \rho(t)}{t-z} .
$$

The spectral function $d \rho(t)$ does not have a bounded variation globally, on the whole real axis, and integral (13.47) diverges in the classical sense. We want to reduce it to a convergent integral by subtracting the classically divergent part of it.

If $q(x)=0$, then $\rho:=\rho_{0}(t)$ for $t<0, m(\sqrt{\lambda})=i \sqrt{\lambda}$, and formula (13.44) with $a=0$ yields

$$
\rho_{0}(\lambda)=\frac{2 \lambda^{\frac{3}{2}}}{3 \pi} .
$$

If $q(x)=0$ then $G(x, y, \lambda)=\frac{\sin (\sqrt{\lambda} y)}{\sqrt{\lambda}} e^{i \sqrt{\lambda} x}, \quad y \leq x$, so (13.39) yields $m(\sqrt{\lambda})=$ $i \sqrt{\lambda}$. Formula (13.47) yields formally

$$
i \sqrt{\lambda}=\frac{1}{\pi} \int_{0}^{\infty} \frac{\sqrt{t} d t}{t-\lambda}
$$

This integral diverges from the classical point of view. Let us interpret (13.49) as follows. Let $\operatorname{Im} \lambda>0$. Differentiate (13.49) formally and get

$$
\frac{i}{2 \sqrt{\lambda}}=\frac{1}{\pi} \int_{0}^{\infty} \frac{\sqrt{t} d t}{(t-\lambda)^{2}}, \quad \operatorname{Im} \lambda>0 .
$$

This is an identity, so (13.49) can be interpreted as an integral from 0 to $\lambda$ of (13.50). The integral $\int_{0}^{\infty} t^{-\frac{1}{2}} d t$ which one obtains in the process of integration, is interpreted as zero, as an integral of a hyperfunction or Hadamard finite part integral.

Subtract from (13.47) the divergent part (13.49) and get:

$$
m(\sqrt{z})-i \sqrt{z}=\int_{-\infty}^{\infty} \frac{d \sigma(t)}{t-z}
$$

where

$$
d \sigma(\lambda)=d \rho(\lambda)-d \rho_{0}(\lambda), \quad d \rho_{0}(\lambda):= \begin{cases}\frac{\sqrt{\lambda} d \lambda}{\pi}, & \lambda \geq 0, \\ 0, & \lambda<0 .\end{cases}
$$

Integral (13.47') converges in the classical sense if $q \in L_{1,1}\left(\mathbb{R}_{+}\right)$. Indeed, by (2.5) and $\left(13.47^{\prime \prime}\right)$ one has $d \sigma(t)=\frac{\sqrt{t}}{\pi}\left(\frac{1}{\mid f\left(\left.\sqrt{t}\right|^{2}\right)}-1\right) d t$. By $(5.65)$ one has $f(\sqrt{t})=1+$ 
$O\left(\frac{1}{\sqrt{t}}\right)$ as $t \rightarrow+\infty$. Thus $d \sigma(t)=O\left(\frac{1}{\sqrt{t}}\right) d t$ as $t \rightarrow+\infty$. Therefore integral (13.47') converges in the classical sense, absolutely, if $\operatorname{Im} z \neq 0$, otherwise it converges in the sense of the Cauchy principal value.

Let us write (11.1) as

$m(k)-i k=\int_{-\infty}^{\infty} e^{i k t}\left[-\sum_{j=0}^{J} r_{j} e^{k_{j} t} H(-t)+a(t) H(t)\right] d t, \quad H(t)= \begin{cases}1, & t \geq 0 \\ 0, & t<0 .\end{cases}$

From (13.51) and (13.47') one gets

where

$$
\int_{-\infty}^{\infty} \frac{d \sigma(s)}{s-\lambda}=\int_{-\infty}^{\infty} e^{i k t} \alpha(t) d t, \quad \lambda=k^{2}+i 0
$$

$$
\alpha(t):=-\sum_{j=0}^{J} r_{j} e^{k_{j} t} H(-t)+a(t) H(t) .
$$

Taking the inverse Fourier transform of (13.52) one can find $\alpha(t)$ in terms of $\sigma(s)$. If $k>0$ then $k=\sqrt{k^{2}+i 0}$ and if $k<0$ then $k=\sqrt{k^{2}-i 0}$. Thus:

$$
\begin{gathered}
\alpha(t)=\frac{1}{2 \pi} \int_{-\infty}^{\infty} d k e^{-i k t} \int_{-\infty}^{\infty} \frac{d \sigma(s)}{s-k^{2}-i 0} \\
=-\frac{1}{2 \pi} \int_{-\infty}^{\infty} d \sigma(s)\left[\int_{0}^{\infty} d k \frac{e^{-i k t}}{k^{2}+i 0-s}+\int_{-\infty}^{0} d k \frac{e^{-i k t}}{k^{2}-i 0-s}\right] .
\end{gathered}
$$

Let us calculate the interior integral in the right-hand side of the above formula. One has to consider two cases: $s>0$ and $s<0$. Assume first that $s>0$. Then

$$
\begin{aligned}
& \int_{0}^{\infty} d k \frac{e^{-i k t}}{k^{2}+i 0-s}+\int_{-\infty}^{0} d k \frac{e^{-i k t}}{k^{2}-i 0-s}=\int_{-\infty}^{\infty} \frac{e^{-i k t} d k}{k^{2}-s}+i \pi\left[\int_{0}^{\infty} e^{-i k t} \delta\left(k^{2}-s\right) d k-\right. \\
&\left.-\int_{0}^{-\infty} e^{-i k t} \delta\left(k^{2}-s\right) d k\right]=-\frac{\pi}{\sqrt{s}} \sin (\sqrt{s} t) H(s)+J
\end{aligned}
$$

where

If $s<0$, then

$$
J:=\int_{-\infty}^{\infty} \frac{e^{-i k t} d k}{k^{2}-s}
$$

$$
J=\frac{\pi}{\sqrt{|s|}} e^{-\sqrt{|s|}|t|}
$$

If $s>0$, then

$$
J=-\frac{\pi}{\sqrt{s}} \sin (|t| \sqrt{s}) .
$$

From (13.54)-(13.57) one gets

$$
\alpha(t)=\int_{0}^{\infty} d \sigma(s) \frac{\sin (t \sqrt{s})}{\sqrt{s}} H(t)-\frac{1}{2} \int_{-\infty}^{0} \frac{d \sigma(s)}{\sqrt{|s|}} e^{-|t| \sqrt{|s|}} .
$$

Formula (13.58) agrees with (13.53): the second integral in (13.58) for $t>0$ is an $L^{1}\left(\mathbb{R}_{+}\right)$function, while for $t<0$ it reduces to the sum in (13.53) because $d \sigma(s)=d \rho(s)$ for $s<0, d \rho(s)$ for $s<0$ is given by formula (2.5) and the relation between $c_{j}$ and $r_{j}$ is given by formula (2.7). 


\section{References}

[1] Airapetyan, R., Ramm, A. G., Smirnova, A. B. [1999] Example of two different potentials which have practically the same fixed-energy phase shifts, Phys. Lett. A., 254, N3-4, pp.141-148.

[2] Borg, G. [1946] Eine Umkehrung der Sturm-Liouvilleschen Eigenwertaufgabe, Acta Math., 78, N1, pp. 1-96.

[3] Chadan, K., Sabatier, P. [1989] Inverse problems in quantum scattering theory, Publisher Springer-Verlag, New York.

[4] Del Rio, R., Gesztesy, F., Simon, B. [1997] Inverse spectral analysis with partial information on the potential III, Int. Math. Res. Notices, 15, pp.751-758.

[5] Denisov, A. [1994] Introduction to the theory of inverse problems, Moscow Univ., Moscow.

[6] Gakhov, F. [1966] Boundary value problems, Publisher Pergamon Press, New York.

[7] Gradshteyn, I., Ryzhik, I. [1985] Tables of integrals series and products, Publisher Acad. Press, New York.

[8] Gelfand, I., Raikov, D., Shilov, G. [1964] Commutative normed rings, Publisher Chelsea, New York.

[9] Gesztesy, F., Simon, B., [1998] A new approach to inverse spectral theory II, (1998 preprint).

[10] Levin, B. [1980] Distribution of zeros of entire functions, AMS Transl. vol.5, Providence, RI.

[11] Levitan, B. [1987] Inverse Sturm-Liouville problems, Publisher VNU Press, Utrecht.

[12] Levitan, B. [1964] Generalized translation operators, Jerusalem.

[13] Levitan, B. [1994] On the completeness of the products of solutions of two SturmLiouville equations, Diff. and Integr. Eq., 7, N1, pp.1-14.

[14] Marchenko, V. [1986] Sturm-Liouville operators and applications, Publisher Birkhüser, Boston.

[15] Marchenko, V. [1994] Characterization of the Weyl's solutions, Lett. Math-Phys, 31, pp.179-193.

[16] Newton, R. [1982] Scattering theory of waves and particles, Publisher Springer-Verlag, New York.

[17] Ramm, A. G. [1992] Multidimensional inverse scattering problems, Publisher Longman Scientific \& Wiley, New York, pp.1-379. [1994] Expanded Russian edition, Mir, Moscow, pp.1-496.

[18] [1999] Property C for ODE and applications to inverse scattering, Zeit. fuer Angew. Analysis, 18, N2, pp.331-348.

[19] [1987] Recovery of the potential from I-function, Math. Reports of the Acad. of Sci., Canada, 9, pp.177-182.

[20] [1998] A new approach to the inverse scattering and spectral problems for the Sturm-Liouville equation, Ann. der Phys., 7, N4, pp.321-338.

[21] Inverse problem for an inhomogeneous Schrödinger equation, Jour. Math. Phys., 40, N8, (1999), 3876-3880.

[22] _ [1998] Recovery of compactly supported spherically symmetric potentials from the phase shift of s-wave, In the book: Spectral and scattering theory, Plenum publishers, New York, (ed. A.G.Ramm), pp.111-130.

[23] [1999] Inverse scattering problem with part of the fixed-energy phase shifts, Comm. Math. Phys., 207, N1, (1999), 231-247.

[24] [1992] Stability estimates in inverse scattering, Acta Appl. Math., 28, N1, pp.142.

[25] _ [1987] Characterization of the scattering data in multidimensional inverse scattering problem, in the book: "Inverse Problems: An Interdisciplinary Study." Publisher Acad. Press, New York, pp.153-167. (Ed. P.Sabatier).

[26] [1986] On completeness of the products of harmonic functions, Proc. A.M.S., 99, pp.253-256.

[27] [1987] Completeness of the products of solutions to PDE and uniqueness theorems in inverse scattering, Inverse problems, 3, pp.L77-L82

[28] [1988] Recovery of the potential from fixed energy scattering data, Inverse Problems, 4, pp.877-886; 5, (1989) p.255. 
[29] [1988] Multidimensional inverse problems and completeness of the products of solutions to PDE, J. Math. Anal. Appl. 134, 1, pp.211-253; 139, (1989) 302.

[30] [1990] Completeness of the products of solutions of PDE and inverse problems, Inverse Probl.6, pp.643-664.

[31] _ [1991] Necessary and sufficient condition for a PDE to have property C, J. Math. Anal. Appl.156, pp.505-509.

[32] [1991] Symmetry properties for scattering amplitudes and applications to inverse problems, J. Math. Anal. Appl., 156, pp.333-340.

[33] [1981] Stable solutions of some inverse and ill-posed problems, Math. Meth. in appl. Sci. 3, pp.336-363.

[34] [1990] Random fields estimation theory, Publisher Longman Scientific and Wiley, New York

[35] [1966] Some theorems on equations with parameters in Banach spaces, Doklady Acad. Sci. Azerb. SSR, 22, pp.3-6.

[36] [1988] Inverse scattering on half-line, J. Math. Anal. Appl.,133, N2, pp.543-572.

[37] Ramm, A. G., Arredondo, J. H., Izquierdo, B. G., [1998] Formula for the radius of the support of the potential in terms of the scattering data, Jour. of Phys. A, 31, N1, pp. L39-L44.

[38] Ramm, A. G., Porru, G., [1996] Completeness and non-completeness results for the set of products of solutions to differential equations, Applicable Analysis, 60, pp.241-249.

[39] Ramm, A. G., Scheid, W., [1999] An approximate method for solving inverse scattering problem with fixed-energy data, Journ. of Inverse and Ill-Posed Probl., 7, N6, 561-571.

[40] Ramm, A. G., Smirnova, A., [2000] A numerical method for solving the inverse scattering problem with fixed-energy phase shifts, Journ. of Inverse and Ill-Posed Probl., 8, N3, (2000), 307-322.

[41] Rudin, W. [1974] Real and complex analysis, Publisher McGraw Hill, New York.

[42] Rundell, W., Sacks, P. [1992] Reconstruction techniques for classical Sturm-Liouville problems, Math. Comput., 58, pp.161-183.

[43] Volk, V. [1953] On inverse formulas for a differiential equation with a singularity at $x=0$, Uspekhi Mat. Nauk, 8, N4, pp.141-151. 